\title{
VIII. Beiträge zur Kenntniss des Pyrargyrit und Proustit*).
}

\author{
Von \\ H. A. Miers in London mit Analysen von G. T. Prior.
}

(Mit Tafel IV, V und 1 Holzschnitt.)

\section{\$1. Historische Uebersicht.}

Von einer vollständigen historischen Entwickelung unserer Kenntnisse uber die Rothgiltigerze, sowie einer Zusammenstellung der Literatur und der beobachteten Formen kann hier Abstand genommen werden, da dies bereits von Rethwisch in seiner Arbeit: "Beiträge zur mineralogischen und chemischen Kenntniss des Rothgiltigerzes “**) geschehen ist. Unter Berucksichtigung der weiter unten discutirten Fehler und Irrthumer kann denn diese Arbeit als Uebersicht unserer krystallographischen und chemischen Kenntnisse uber die Rothgiltigerze bis zum Jahre 1886 dienen.

a) In früheren Arbeiten mangelt es manchmal an präcisen Beobachtungen uber die beiden Mineralien; nur wenig Analysen wurden mit sorgfältig gepruftem Material angestellt, und die Angaben von competenten Beobachtern uber den Werth des Rhomboëderwinkels schwanken beim Pyrargyrit von $71^{\circ} 18^{\prime}$ bis $71042^{\prime}$ und beim Proustit von 720 10' bis $72^{\circ} 45^{\prime}$. Den schon zahlreichen, beobachteten Formen fehlt die kritische Discussion und sichere Angaben uber ihre Zugebörigkeit zum Proustit oder Pyrargyrit. Sella, welcher zahlreiche neue Formen zuerst aufführt, scheint sich seinerzeit mit dem Gedanken einer monographischen Bearbeitung dieses Gegenstandes getragen zu haben; seine diesbezüglichen zahlreichen Beobachtungen, welche er in den verschiedenen europäischen Museen gemacht

*) Gelesen vor der Mineralog. Gesellsch. zu London am 8. Mai 1888. A. d. Min. Mag. 8, $37 \mathrm{~m}$. Verbesserungen u. Zusälzen v. Verf. mitgeth.

**) N. Jahrb. f. Min. etc. 1886, Beil.-Bd. 4, 31-109. Hef. in dieser Zeitschr. 12, 67 f. Groth, Zeitachrift f. Krystallogr. XV. 
hatte, wurden jedoch nie publicirt, und er begnügte sich mit der Wiedergabe einer Formentabelle.

Von den intermediären Varietäten des Rothgiltigerzes, d. h. denen, welche sowohl Antimon als auch Arsen enthalten, nahm man an, dass sie bezuglich ihrer Farbe, sowie der Grösse ihres Rhomboëderwinkels zwischen den beiden Endgliedern Pyrargyrit und Proustit ständen.

Aus dem Auftreten von $\{10 \bar{T} 0\} \infty R$ als trigonales Prisma, sowie aus dem Habitus der nur sehr selten an beiden Enden ausgebildeten Krystalle hatte man zwar den Schluss gezogen, dass der Pyrargyrit hemimorph sei, man war jedoch nicht im Stande, die beiden Enden und ihre charakteristischen Formen zu unterscheiden.

Zwillingskrystalle nach $\left\{10 T_{4}\right\}_{4}^{1} R$ wurden von Haidinger*) schon vollständig beschrieben; ferner liegen Beschreibungen von Zwillingen nach $\{10 T 1\} R$ vom Pyrargyrit und Proustit und solcher nach $\{0001\} 0 R$ vom Pyrargyrit vor. Zwillinge nach $\{01 \bar{T} 2\}-\frac{1}{2} R,\{10 \bar{T} 0\} \infty R$ und $\{02 \overline{2} 1\}-2 R$ wurden von verschiedenen Autoren beobachtet. - Bezüglich der nur sehr unvollständigen Beobacbtungen über die physikalischen Eigenschaften, sowie der früberen chemischen und krystallographischen Arbeiten sehe man die Arbeit von Rethwis ch nach.

Der von Letzterem gegebenen Literaturzusammenstellung ist noch einzufugen :

1795. Freiesleben, Bemerkungen über den Harz (passim).

1811. S owerby, Exotic Mineralogy. 1. Plate XXXIII.

1824-25. Haid ing er, Edinburgh Journal of Seience, 1, $326 ; 2,94$.

1836. Mo h s, Naturgeschichte 1, 252.

1846. Do meyko, Annales des Mines 9, 365.

1851. Sénarmont, Annales de Chimie et de Physique 32, 171.

1853. Breith aup t, Berg- und Hültenmännische Zeitung 12; 401.

1856. Vogl, Gangverhältnisse und Mineralreichthum Joachimsthals S. 81.

Sella, Mem. Accademia Torino 17, LXIX. Nuovo Cimento 4, 93.

1863. Nöggerath, Ber. niederrheinische Gesellschaft $20,51$.

Ih ne, Berg- und Hüttenmännische Zeitung 22, 51.

1882. Da ubrée, Bull. Sociélé minéralogique de France 5, 300.

vom Rath, Ber. niederrheinische Gesellschaft, S. 34 .

1885. Schenck, diese Zeitschr. 10, 283.

1886. Rethwisch, Neues Jahrbucb, Beil.-Bd. 4, 31.

Slreng, Neues Jahrbuch (I), 60.

Schuster, Verh. d. geolog. Reichsanstalt, S. 68 .

1887. Schuster, diese Zeitschr. 12, 117.

Goldschmidt, Krystallographische Projectionsbilder $5,6$.

Purgold, Isis, 1886, S. 53. Ref. diese Zeitschr. 14, 404.

1888. Mi ers and Prior, Mineralogical Magazine 7, 196 und diese Zeitschr. 14, 113.

Goldschmidt, Index der Krystallformen.

*) Edinburgh Journ. Sc. 1825, 2, 91. 
b) Die Resultate der neueren Arbeiten (seit 1886). Rethwisch theilt Untersuchungen und Messungen mit, welche er an vier Exemplaren ausgeführt hat, von denen eines ein reiner Proustit, ein zweites reiner Pyrargyrit und die beiden anderen 2,62 beziehungsweise 3,01\% Arsen enthaltende Pyrargyrite sind. Sein Hauptaugenmerk war auf die Bestimmung des Rhomboëderwinkels der beiden ersten gerichtet und ist er wohl der Erste, welcher gemessene Krystalle dieses Minerals zur chemischen Untersuchung verwendete, um eine präcisere Grundlage für die Beziehungen zwischen Krystallform und chemischer Zusammensetzung derselben zu gewinnen.

Auf den ersten Blick scheinen diese vier Exemplare bezuglich des Rhomboëderwinkels, des Arsengehaltes und des specifischen Gewichtes eine forllaufende Reihe zu bilden. Leider sind jedoch die beiden $Z$ wischenglieder, obwohl von verschiedenem Fundort, in Wirklichkeit fast so gut wie identisch, und die Dimensionen des Pyrargyrits sind nicht hinreichend befriedigend bestimmt in Bezug auf Genauigkeit der Messungen, als deren Uebereinstimmung. In der That können, wie weiterhin gezeigt werden soll (vergl. auch diese Zeitschr. 14, 115) diese Messungen zu dem Schlusse fuhren, dass dem reinen Pyrargyrit derselbe Rhomboëderwinkel zukomme, wie den beiden Arsen-haltigen Varietäten.

Die Beobachtungen von $R$ el $\mathrm{h} w$ is $\mathrm{ch}$ sind deshalb nicht hinreichend, um einen allmählichen Uebergang von Pyrargyrit zu Prouslit zu beweisen; ebenso wenig ergaben sie mit genügender Sicherheit die Dimensionen des Pyrargyrit. Thatsäehlich folgt aus ihnen nur: 1) Ein Exemplar von Proustit hatte den Rhomboëderwinkel von 720 10' und das specifische Gewicht 5,55. 2) Zwei Pyrargyritexemplare mit $3 \%$ Arşen hatten den Rhomboëderwinkel $71022^{\prime}$ und das specifische Gewicht 5,72 bis 5,75. 3) Ein Exemplar reinen Pyrargyrits besass den Rhomboëderwinkel von vielleicht etwas weniger als 710 22' und das specifische Gewicht 5,8\%.

Die krystallographischen Daten dieser Arbeil werden weiter unten $(\S 9)$ discutirt.

M. Schuster lenkt die Aufmerksamkeit auf die Streifung der Prismenflächen $\{1 \mid \overline{2} 0\} \infty P 2$ des Pyrargyrits, welche auf verschiedene Formen an den beiden Enden der Krystalle fuhrt und den hemimorphen Charakter derselben beweist. Ferner zeigt er, wie die Hemimorphie durch eine eigenthumliche Art von Zwillingsbildung *) verdeckt werden kann.

An den beiden von ihm beschriebenen Krystallen von Andreasherg treten am aufgewachsenen Ende spitze negative und flache positive Skale-

*) Schuster scheint nicht bekannt gewesen zu sein, dass diese Zwillingsbildung bereits von Na umann (Lehrb. d. Krystallogr. 1830, 2, 311, Fig. 721) beschrieben und abgebildet wurde. 
noëder und am frei ausgebildeten Ende spitze positive und flache negative Skalenoèder auf. Die Art der Zwillingsbildung ist eine unsymmetrische und erklärbar durch Hemitropie um die Normale der Prismenflächen $\{1 \mid \overline{2} 0\} \infty P 2$. Die beiden Individuen sind derartig verwachsen, dass die Prismenflächen zusammenfallen und ein scheinbar einfaches Individuum bilden, in dessen Mitte sich die beiden Enden mit den charakteristischen negativen Skalenoëdern begegnen. In einem doppelendigen Krystalle eines derartigen Zwillings tritt die Hemimorphie nicht hervor, indem beide Enden gleich erscheinen, und nur durch sorgfältige Beobachtung der Prismenstreifung und ihrer Richtung können die Zwillingsnatur und die Verwachsungsgrenzen erkannt werden.

Die letzte Arbeit über diesen Gegenstand, eine Notiz von G. T. P rior und mir*) ergab, dass ein wenigstens 1,41\% Antimon enthaltender Proustit vom specifischen Gewicht 5,64 einen Rhomboëderwinkel besitzt, welcher gleich dem des reinen Proustit, nämlich gleich 720 12' ist.

V. Goldschmidt schliesslich (in seinen Projectionsbildern) entnahm der Hethwisch'schen Zusammenstellung die ihm als sicher festgestellt erscheinenden Formen, zeigt in ausgezeichneten gnomonischen Projectionen deren Vertheilung in Zonen und vergleicht dieselben mit den Formen des Kalkspaths und Quarzes. In seinem Index der Krystallformen kommt er nach eingebender Discussion der früberen Beobachtungen zu dem Schlusse, dass die gleichmässige Entwickelung der von den Prismen $\{11 \overline{2} 0\} \infty P 2$ und $\{10 \bar{T} 0\} \infty R$ ausstrahlenden Zonen als charakteristisch fur das Rothgiltigerz angesehen werden kann (s. unten $§ 22$ ).

\section{§ 2. Resultate.}

Die vorliegende Arbeit ist das Resultat einer Untersuchung des reichen Materials des British Museum. Die Analysen wurden von G. T. Prior, das specifische Gewicht in den meisten Fällen von G. T. Prior und mir bestimmt. Die Resultate, zu denen wir gelangten, sind folgende :

1) Proustit und Pyrargyrit sind zwei stets zu unterscheidende Species.

2) Proustit hat den Rhomboëderwinkel 720 12', das specifische Gewicht 5,57; Pyrargyrit hat den Rhomboëderwinkel 710 22' und das specifische Gewicht 5,85.

3) Die beiden Mineralien können vollkommen durch die Farbe ihres Pulvers unterschieden werden. Das des Proustit ist scharlachzinnoberroth, das des Pyrargyrit purpurroth.

4) Pyrargyrit ist zweifellos, Proustit wahrscheinlich hemimorph.

*) Diese Zeitschr. 14, 113. Der Fundort des S. 115 erwăhnten Proustit ist nicht Chañarcillo, sondern Mexico. 
5) Von den Formen sind einige dem Proustit, andere dem Pyrargyrit eigenthümlich, während eine Anzabl beiden gemeinsam sind. Diese werden weiterhin unterschieden.

6) Von den Formen des Pyrargyrit sind einige dem oberen, andere dem unteren Ende des Krystalles eigenthümlich, andere sind beiden Enden gemeinsam. Dieselben werden weiter unten ebenfalls, soweit es möglich, getrennt.

7) Die meisten Pyrargyrite enthalten Arsen und einige Proustite Antimon. Solche Exemplare liefern nur selten genau messbare Krystalle; ist der Procentgehalt ein beträchtlicherer, so wird er gewöbnlich durch eine erkennbare Verwachsung von Pyrargyrit und Proustit bewirkt und veranlasst eine wirre Krystallisation.

8) In den Fällen, in welchen die Beimengung nur gering ist und die Krystalle gut messbar sind, bringt das Vorhandensein von Antimon in Proustit und von Arsen in Pyrargyrit keine merkliche Veränderung im Rhomboëderwinkel hervor.

9) Der Pyrargyrit bildet gesetzmässige Zwillinge nach $\{10 \bar{T} 4\}_{\frac{1}{4}} R$, $\{10 \bar{T} 1\} R,\{11 \overline{2} 0\} \propto P 2,\{01 \bar{T} 2\}-\frac{1}{2} R$ und nach $\{0001\} 0 R$, der Proustit nach $\{10 T 4\} \frac{1}{4} R,\{10 \bar{T} 1\} R,\{0001\} 0 R,\{01 \bar{T} 2\}-\frac{1}{2} R$. Zieht man die Hemimorphie des Pyrargyrit in Betracht, so sind die Zwillinge nach $\{10 T 4\} \frac{1}{4} R$ und $\{10 T 1\} R$ stets durch Hemitropie nach diesen Flächen erklärlich. Ferner ist $\{10 \pi 4\} \frac{1}{4} R$ Ebene secundärer Zwillingsbildung beim Pyrargyrit.

10) Die Hauptzonen des Pyrargyrit sind sehr reich an Vicinalflächen und Formen mit hohen Indices. Diese Zonen werden bestimmt durch die Rhomboẹder $\{01 T 2\}-\frac{1}{2} R,\{10 T 1\} R$ und die Prismen $\{11 \overline{2} 0\} \infty P 2,\{10 \bar{T} 0\} \infty R$. Die Vicinal 0 ächen besitzen Positionen, welche als regelmässige Functionen der Indices jener ausgedrück $\iota$ werden können.

11) Am reichsten daran sind jene Theile der Hauplzonen, wo sie sich am meisten denjenigen Flächen der Formen $\{10 \bar{T} 1\} R,\{11 \overline{2} 0\} \infty P 2$ und $\{10 T 0\} \infty R$ nähern, welche nicht in der betreffenden Zone liegen.

12) Alle typischen Formen gehören zu Zonen mit einfachen Symbolen, welche entweder $\{10 T 1\} R$ oder $\{01 T 2\}-\frac{1}{2} R$ enthalten.

13) Die beiden Mineralien sind streng rhomboëdrisch und keine der typischen Formen tritt in beiden ( + und -) Stellungen auf.

Die rein mineralogischen Angaben und Details werden in dieser Beschreibung nicht gegeben; sie werden in dem ausfuhrlichen Catalog der Sammlung des British Museum mitgetheilt werden. 


\section{§ 3. Beschreibung des Pyrargyrit und Proustit.}

Pyrargyrit. - Rhomboëdrisch, hemimorph.

$$
a: c=1: 0,7892 \text {. }
$$

$R: R=(10 \overline{1} 1):(\bar{T} 101)=71022^{\prime} ; \quad R: 0 R=(10 \overline{1} 1):(0001)=42^{0} 20 \frac{1}{2}^{\prime}$.

Beobachlete Formen:

$a\{11 \overline{2} 0\} \infty P P 2$
$b\{10 \overline{1} 0\} \infty 0 R$
$c\{13 \overline{7} 1\} R 7$
$d\{12 \overline{3} 2\}-\frac{1}{2} R 3$
$e\{01 \overline{1} 2\}-\frac{1}{2} R$
$f\{05 \overline{5} 1\}-5 R$
$? g\left\{\{1 \overline{3} 2\} \frac{1}{2} R 3\right.$
$? k\{10.5 . \overline{1} \overline{5} .8\} \frac{5}{8} R 3$
$l\{45 \overline{9} 5\}-15$
$n\{41 \overline{5} 3\} R 3$
$? 0\{0001\} 0 R$
$p\{11 \overline{2} 3\} \frac{2}{3} P 2$
$q\{16 \overline{7} 1\}-5 R \overline{5}$
$r\{10 \overline{1} 1\} R$
$s\{02 \overline{2} 1\}-2 R$
$t\{21 \overline{3} 4\} \frac{1}{4} R 3$
$u\{10 \overline{1} 4\} \frac{1}{4} R$
$v\{21 \overline{3} 1\} R 3$
$w\{31 \overline{4} 5\} \frac{2}{5} R 2$
$y\{32 \overline{5} 1\} R 5$
$z\{43 \overline{7} 4\} \frac{1}{4} R 7$
$a^{\prime}\{53 \overline{8} 8\} \frac{1}{4} R 4$

\begin{tabular}{|c|}
\hline$f^{\prime}\{31 \overline{4} 0\} \infty \mathrm{H} 2$ \\
\hline$g^{\prime}\{11.5 . \overline{1} \overline{6} .9\} \frac{2}{3} R_{3}^{8}$ \\
\hline$m^{\prime}\{6.5 . \overline{11} .7\} \frac{1}{7} R 11$ \\
\hline$p^{\prime}\{17.1 . \overline{1} \overline{8} .1\} \mathcal{A}_{6} R_{\mathrm{8}}^{9}$ \\
\hline$r^{\prime}\{50 \overline{5} 6\}+\frac{5}{6} R$ \\
\hline$s^{\prime}\{7.3 . \overline{1} 0.4\} R_{2}^{\frac{5}{2}}$ \\
\hline$t^{\prime}\{14.11 .25 .15\} \frac{1}{5} R_{3}^{25}$ \\
\hline$w^{\prime}\{51 \overline{6} 4\} R \frac{3}{2}$ \\
\hline$x^{\prime}\{7.4 . \overline{11} .8\} \frac{3}{4} R \frac{11}{3}$ \\
\hline$B\{4.3 . \overline{7} .6\} \frac{1}{6} R 7$ \\
\hline$C\{11.4 . \overline{5} .10\}_{10}^{7} R_{7}^{15}$ \\
\hline$\left.D\{1.12 . \bar{\top} .1\}-11 R_{1}^{1}\right\}^{3}$ \\
\hline$E\{13 \overline{4} 1\}-2 R z$ \\
\hline$F\{4.15 . \overline{4} \overline{9} .4\}-\frac{11}{4} R_{1}^{1} \frac{9}{1}$ \\
\hline$G\{1.9 . \overline{1} \overline{0} .1\}-8 R^{5}$ \\
\hline$H\{15 \overline{6} 1\}-4 R \frac{3}{2}$ \\
\hline$I\{50 \overline{58}\} \frac{5}{8} R$ \\
\hline$L\{53 \overline{8} 6\} \frac{1}{3} R 4$ \\
\hline$N\{2.9 . \overline{1} .2\}-\frac{7}{2} R_{9}^{1} 1$ \\
\hline$P\{15 \overline{6} 2\}-2 R_{2}^{3}$ \\
\hline$Q\{14.4 . \overline{1} 8.13\} \frac{10}{13} R_{5}^{9}$ \\
\hline$S\{2.13 . \overline{15} .7\}-\frac{11}{7} R_{1.5}$ \\
\hline
\end{tabular}

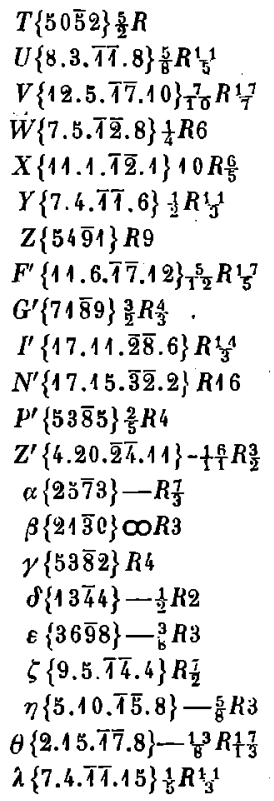

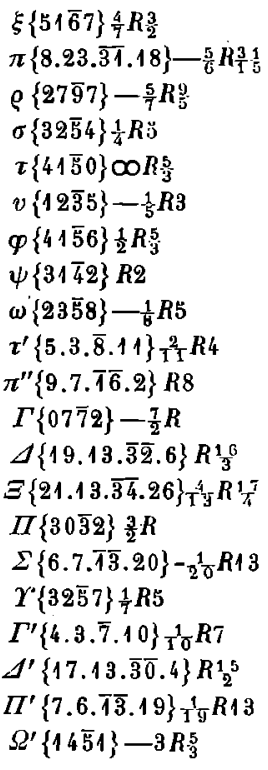

Zwillinge nach (1) $u,(2) r$, (3) $a,(4) e,(5) b(?)$. Spaltbarkeit nach $r$, unvollkommen nach $e$. Bruch muschelig.

Härte 2, 5, spec. Gew. 5,85. In den Arsen-haltigen Varietäten von 5,77 bis 5,85 schwankend. Die Farbe ist im reflectirten Lichte schwarz bis grauschwar', im durchfallenden Lichte röthlich-purpurn, Strich- und Pulverfarbe purpurroth. Durchscheinend bis undurchsichtig. Metallartiger Diamantglanz.

Zusammensetzung $3 \mathrm{Ag}_{2} \mathrm{~S} . \mathrm{Sb}_{2} \mathrm{~S}_{3}$, im Allgemeinen einen geringen Procentsatz Arsenik enthaltend.

Proustit. - Rhomboëdrisch; wahrscheinlich hemimorph.

$$
a: c=1 ; 0,8038 \text {. }
$$
$R: R=(10 \pi 1):(\bar{T} 101)=72012 ;$
$R: 0 R:(10 T 1):(0001)=42^{0} 52^{\prime}$. 
Beobachlete Formen:

\begin{tabular}{|c|c|c|c|}
\hline$a\{11 \overline{2} 0\} \infty P\}$ & $r\{10 \overline{1} 1\} R$ & $n^{\prime}\{8.3 . \bar{\top} .2\} \frac{5}{2} R^{U}$ & $\vartheta^{\prime}\{52 \overline{7} 9\} \frac{1}{3} A_{\frac{7}{3}}$ \\
\hline$b\{10 \bar{T} 0\} \infty R$ & $s\{02 \overline{2} 1\}-2 R$ & $M\{35 \overline{8} 7\}-\frac{2}{7} R 4$ & $\mathcal{I}\{19.13, \overline{3} \overline{2} .6\} R^{\prime}{ }^{6}$ \\
\hline$d\{12 \overline{3} 2\}-\frac{1}{2} R 3$ & $t\{21 \overline{3} 4\} \frac{1}{4} R 3$ & $P\{15 \overline{6} 2\}-2 R_{2}^{3}$ & $\Phi\{11.5 . \overline{1} \overline{6} .12\} \frac{1}{2} H^{8}$ \\
\hline$e\{01 \bar{T} 2\}-\frac{1}{2} R$ & $u\{10 \bar{T} 4\} \frac{1}{4} R$ & $\alpha\{28 \overline{7} 3\} \longrightarrow R \frac{7}{3}$ & $\Psi\{43 \overline{7} 7\} \frac{1}{7} R 7$ \\
\hline $9 h\{03 \overline{3} 2\}-\frac{3}{2} R$ & $v\{21 \overline{3} 1\} R 3$ & $\gamma\{53 \overline{8} 2\} R 4$ & \\
\hline $\begin{array}{l}P \circ\{0001\} 0 R \\
p\{11 \overline{2} 3\} \frac{2}{3} P 2\end{array}$ & $\begin{array}{l}w\{31 \overline{4} 5\}\}_{5}^{2} R 2 \\
y\{32 \overline{5} 1\} R 5\end{array}$ & $\begin{array}{l}\zeta\{9.5 . \bar{T} T .4\} R \frac{7}{2} \\
\tau\{41 \overline{5} 0\} \infty 0 R \frac{5}{3}\end{array}$ & - \\
\hline
\end{tabular}

Zwillinge nach (1) $u,(2) r,(3) o$, (4)e. Spalıbarkeit nach $r$ unvollkommen. Bruch muschelig. Härte $=2,5 *)$. Spec. Gew. 5,57 , in den Antimonhaltigen Varietäten von 5,58 bis 5,64 schwankend.

Die Farbe ist im reflectirten Lichte schwarz bis röthlich-schwarz, im durchfallenden Lichte zinnoberroth. Strich und Pulverfarbe scharlach-zinnoberroth. Halbdurchsichtig bis durchscheinend. Diamantglanz.

Zusammensetzung $3 \mathrm{Ag}_{2} \mathrm{~S} . \mathrm{As}_{2} \mathrm{~S}_{3}$, zuweilen einen geringen. Procentsatz Antimon enthaltend.

\section{\$4. Farbe und Strich.}

In rein reflectirtem Lichte ist die Farbe beider Mineralien dieselbe, nämlich schwarz oder grauschwarz, und nur bei theilweise durchfallendem Lichte erscheinen dieselben von rother, in den verschiedenen Lehrbuchern mit mannigfachen Namen belegter Farbe. Diese wechselt in verschiedenen Tönen je nach der Dicke der Krystalle, und ganz besonders dann, wenn die Krystalle angelaufen sind. Eine bestimmte Bezeichnung kann nur dann angewendet werden, wenn beide Mineralien in durchfallendem Lichte in so dünnen Splittern oder Schliffen beobachtet werden, dass sie durchscheinę̧d oder halb durchsichtig werden. Unter solchen Umständen zeigt der Pyrargyrit ein röthliches Purpur und der Proustit ein fast dem Zinnober gleiches Roth.

Die Farbe der Mineralien, wie sie bei der gewöhnlichen Beobachtung im theilweise reflectirten, theilweise durchfallenden Lichte erscheint, erlaubt keinen Schluss auf die Zusammensetzung. Proustit wird unter Einwirkung des Lichtes noch schwärzer als Pyrargyrit, und lichte Varietäten des letzteren enthalten manchmal gar kein Arsen. Auch erscheint der Pyrargyrit manchmal heller, wenn er angelaufen oder drusig verwachsen ist, in welch' letzterem Falle die kleineren Krystalle so viel Licht durchlassen, dass sie fast die Oberflächenfarbe des Proustit zeigen. Nur bei der Untersuchung hinreichend dunner Splitter zeigt der Pyrargyrit einen bläulichen Farbenton, den der Proustit niemals zeigt.

Ein genugend sicheres Mittel, um die beiden Mineralien zu unterscheiden, liefert ihr Strich. Derselbe wird ebenfalls von vềrschiedenen

*) Pyrargyrit ist um ein Geringes härter als Proustit. 
Autoren verschieden angegeben; benutzt man jedoch zur Bestimmung der Strichfarbe stets nur ein Splitterchen, welches man mit einem Messer auf weissem Papier verstreicht, so findet man stets nur zwei Strichfarben, nämlich röthlich-purpur für Pyrargyrit und scharlach-zinnober für Proustit.

Auf diese Weise wurden zunächst sämmtliche Rothgiltigerzstücke des British Museum untersucht und provisorisch in zwei Hauptgruppen Pyrargyrite und Proustite getrennt. Hierbei mussten ein Anzahl als Proustit gehende Exemplare zum Pyrargyrit gestellt werden. Alsdann wurden sämmtliche Pyrargyrite auf einen Gehalt an Arsen geprüft und jene, welche eine deutliche Reaction gaben, quantitativ untersucht in der Hoffnung, dadurch auf $\mathrm{Zwischenglieder}$ zu stossen. Die Resultate dieser Untersuchung werden weiter unten mitgetheilt. In keinem Falle enthielt ein Exemplar mit dunklerem Strich mehr als 2,6\% Arsen.

Bei Untersuchung des Striches in der angegebenen Weise tritt kein auffallender Unterschied hervor zwischen dem reinen Pyrargyrit und den 2-3\% Arsen enthaltenden Varietäten; nur wenn eine grosse Menge Substanz angewendet wird, erscheint der Strich der letzteren etwas lichter.

Der Strich des Pyrargyrit ist fast derselbe, wie der bräunlich-carminrothe des Kermesit, nur mit weniger vorwaltendem Braun ; jener des Proustit ist gleich dem des Zinnobers, jedoch mit mehr ziegelrothem Ton an Stelle des rosafarbenen des Zinnobers.

Der Strich des veränderten Proustit, oder der veränderte Strich von frischem Proustit wechselt von ziegelroth bis braunschwarz, kann aber nie mit dem purpurrothen des Pyrargyrit verwechselt werden. Selbst nach zwei und ein halb Jahren konnte ich ohne Schwierigkeit die beiden Striche noch unterscheiden, nachdem sie ununterbrochen dem directen Tages- und Sonnenlichte ausgesetzt waren.

Nach der Chevreul'schen Farbenscala*) ist der Strich des Proustit ungefähr rouge-orange 2, verdunkelt um $\frac{1}{10}$, derjenige des Pyrargyrit violett 4, verdunkelt um ${ }_{7}^{\frac{3}{0}}$.

Nach der Scala von Ridgway (Nomenclature of Colors. Boston 1886) ist : Proustit, scarlet-vermilion, Plate VIl, No. 10,

Pyrargyrit burnt-carmine, Plate VII, No. 1.

Nach Radde's "Internationale Farbenscala (Hamburg 4877) sind:

Proustit im durchfallenden Lichte $1 k$, Strich $30 k$, Pyrargyrit - $\quad-\quad 26 k, \quad-26 f$.

Bei der Untersuchung des Striches auf Papier kommen auch sehr kleine Mengen von Verunreinigungen zum Vorschein; so erkennt man mit grosser Leichtigkeit den schwarzen Strich von Stephanit oder den dunkelkirschrothen des Miargyrit im Contrast mit dem wirklichen Hothgiltigerzstrich.

*) Mém. Ac. Sc. 1861, 33. 


\section{§ 5. Habitus.}

Die Flächen zerfallen in zwei natürliche Gruppen, vämlich in die Seitenflächen und in die Endflächen. Erstere umfassen die zwischen dem Theil $r-s$ der Zone $r s$ gelegenen Flächen und die der Prismenzone, letztere die zwischen der Zone $r s$ und der Basis (einschliesslich) gelegenen Flächen. Der Habitus der Seitenflächen kann sein:

a. Prismatisch mit vorwaltendem Prisma $a\{11 \overline{2} 0\}$. S. Fig. $2-7$ und 11-13. Vergl. Lévy Fig. 22, 24, 30.

b. Flach, durch Vorherrschen von positiven Skalenoëdern aus der Zone $a r$, wodurch der Krystall ein gerundetes Aussehen erhält. Fig. 1. Lévy Fig. 32, 33.

c. Lanzettförmig, durch vorwaltende Skalenoëder der Zone $b r$. Fig. 8, 9 .

d. Skalenoëdrisch, wenn ein positives Skalenoëder vorwaltet. Fig. 10. Lév y Fig. 5, 8, 12, 26.

Der Habitus der Endflächen kann sein:

a. Pyramidal, durch Vorwalten von $t, p$ oder $w$ beim Pyrargyrit und $M$ beim Proustit. Fig. 3, 5, 6, 11, 12. Lé v y 4, 31, 33.

b. Rhomboëdrisch, mit vorwaltendem Rhomboëder $e$ oder $r$. Fig. 4. Lévy Fig. 2, 5, 10, 12, 20.

Der pyramidale Habitus geht zuweilen in den rhomboëdrischen über durch Ausbildung einer Reihe von gestreiften Fläehen von der Zone er (Andreasberg); der rhomboëdrische dagegen verwandelt sich in eine flache, der Basis nahe stehende Endigung durch polysynthetische Entwicklung der Rhomboëder e oder $u$ (Guanaxuato). Vergl. Lévy Figg. 37-39.

\section{§ 6. Bestimmung des Rhomboëderwinkels.}

Zur Bestimmung der Grunddimensionen ist es selbstverständlich nờthig, die verwendbaren Messungen mit grösster Sorgfalt und Strenge auszuwäblen. Eine der häufigsten und glattesten Formen bei Pyrargyrit und Proustit ist $z$. B. das Skalenoëder $v=R 3$. Die stumpfen und die scharfen Kantenwinkel desselben sind für verschiedene Werthe des Rhomboëderwinkels $r: r=R: R=\{10 \bar{T} 1\}:\{T 101\}$ und $e: e=\frac{1}{2} R:-\frac{1}{2} R=\{01 T 2\}$ $:\{1012\}$.

\begin{tabular}{|c|c|c|c|c|c|}
\hline$r r$ & $e \boldsymbol{e}$ & \multicolumn{2}{|c|}{$v v$} & & \\
\hline $71013^{\prime}$ & $41058^{\prime}$ & $35^{0} 11^{\prime}$ & $74022^{\prime}$ & Pyrargyrit - & Rethwiseh. \\
\hline $71 \quad 18$ & 422 & $35 \quad 12$ & $74 \quad 24$ & - & Miller. \\
\hline 7122 & 425 & $35 \quad 12$ & 7425 & - & Miers. \\
\hline 7130 & 4212 & $35 \quad 13$ & $74 \quad 27$ & - & Phillips. \\
\hline 7210 & $42 \quad 44 \frac{1}{2}$ & $35 \quad 17 \frac{1}{2}$ & $74 \quad 38 \frac{1}{2}$ & Proustit - & Rethwisch \\
\hline $72 \quad 12$ & 4246 & $35 \quad 18$ & $74 \quad 39$ & - & Miers. \\
\hline
\end{tabular}


Aus dieser Tabelle ist ohne Weiteres klar ersichtlich, dass irgend welche Aenderung in den Grunddimensionen sich stärker bemerkbar macht in den Winkeln der Rhomboëder als in denjenigen der Skalenoëder und bei letzteren wieder stärker im scharfen als im stumpfen Winkel der Polkante. Die aus den letzteren berechneten Grunddimensionen werden daber sehr unsicher, wenn der gemessene Winkel nicht auf die Minute genau ist. Das Axenverhältniss des reinen Pyrargyrit berechnet nun Rethwisch aus folgenden an einem einzigen Krystalle erhaltenen Daten:

\begin{tabular}{ccccccc} 
& Beobachtet: & \multicolumn{2}{c}{ Grenzwerthe: } & \multicolumn{2}{c}{ Berechnet aus } \\
$v v$ & $35010^{\prime} 47^{\prime \prime}$ & $35012^{\prime} 30^{\prime \prime}-3509^{\prime} 30^{\prime \prime}$ & $35012^{\prime}$ & $35011^{\prime}$ \\
$v v$ & 742521 & 7426 & -742430 & 7425 & 7422 \\
$e v$ & $502123 \frac{1}{2}$ & 5022 & -502030 & 5021 & $5019 \frac{1}{2}$
\end{tabular}

Diese Messungen, denen ausserdem kein besonderes Gewicht beigelegt werden kann, da sie nur an vier Kanten eines Krystalles angestellt werden konnten, zeigen als einziges Resultat nur, dass die Grunddimensionen des reinen Pyrargyrit dieselben sind wie die der Arsen-haltigen Varietät, dass also der Rhomboëderwinkel 71022' ist (nicht $71^{0} 13^{\prime}$ wie $R$ ethwisch angiebt):

Dieser Werth ist nun auch der von mir für den Pyrargyrit angenommene. Er ist abgeleitet aus den Messungen des Rhomboëderwinkels $e: e$ oder $r: r$ an 14 Krystallen, welche von fünf Handstücken stammten, wovon drei von Andreasberg, eins von Freiberg und eins von Guanaxualo stammt. Sämmtliche Exemplare wurden mittelst der Fresenius-Babo' schen Methode als arsenfrei befunden. Zu den Messungen wurden nur jene Krystalle benutzt, bei welchen die Rhomboëderflächen vollkommen scharfe Reflexbilder lieferten und die drei Rhomboëderwinkel an einem und demselben Krystall bis auf eine Minute übereinstimmten. Das Ergebniss ist :

$\begin{array}{cccc}r: r & \text { Zabl der Kanten } & \text { Grenzen von } r: r & \text { Grenzen von } e: e \\ 71022^{\prime} & 42 & 71017^{\prime}-71023^{\prime} & 4201^{\prime}-426^{\prime} .\end{array}$

Jede Messung wurde dreimal repelirt.

Auf dieselbe Weise wurde der Rhomboëderwinkel des Proustit durch Messung der Kante $e: e$ an 22 Krystallen ermittelt. Dieselben waren zehn Stufen entnommen, von denen drei von Freiberg, zwei von Mexico und fünf von Chañarcillo stammten.

$\begin{array}{cccc}r: r & \text { Zahl der Kanten } & \text { Grenzen von } r: r & \text { Grenzen von } e: e \\ 72^{0} 12^{\prime} & 66 & 72^{\circ} 8^{\prime}-72^{0} 13^{\prime} & 42^{0} 43^{\prime}-42^{0} 47^{\prime}\end{array}$

Eines dieser Exemplare, welches 1,4\% Antimon enthielt, wurde schon früber (d. Zeitschr. 14, 113) beschrieben, die anderen sind wahrscheinlich fast Anlimon-frei. 
Individuelle Schwankungen, denen die gemessenen Winkel unterworfen sind, zeigen sich so beträchtlich, dass einzig die obige Methode zu sicherem Ziele führt. Uebereinstimmende Messungen von Winkeln, welche gegenuber Veränderungen in den Krystallelementen (wie 2. B. der Winkel $v: v=35^{0} 13^{\prime}$ oben) nicht empfindlich sind, haben nur ganz geringen Werth.

Ueber weitere Bestimmungen siehe $\S 22$.

Un regelmässige Schwankungen gemessener Winkel. Bei dem Versuch, den Rhomboëderwinkel an einem einzelnen Krystall durch directe Messungen genau zu bestimmen, entsteht eine Scbwierigkeit schon dadurch, dass der Winkel, welchen die drei Flächen mit einander bilden, selten derselbe ist. Schwankungen von 4-5 Minuten sind nicht ungewöhnlich, selbst wenn die Flächen vollkommen glalt und glänzend sind.

So zeigten z. B. drei sehr vollkommene Proustitkrystalle, von Chañarcillo, welche von einem und demselben Stuck stammten, folgende Werthe:

I.

\begin{tabular}{|c|c|}
\hline$e_{1} e_{2}$ & $42039^{\prime} 50^{\prime \prime}$ \\
\hline$e_{2} e_{3}$ & $\begin{array}{lll}42 & 40 & 3\end{array}$ \\
\hline$e_{3} e_{1}$ & $42 \quad 48 \quad 27$ \\
\hline . & $7^{\prime \prime}$ \\
\hline
\end{tabular}

II.

\begin{tabular}{lll}
$42^{\circ} 40^{\prime} 17^{\prime \prime}$ & $42^{\circ} 43^{\prime} 13^{\prime \prime}$ \\
424113 & 424347 \\
424730 & 424437 \\
\hline $42043^{\prime} 0^{\prime \prime}$ & & $42^{\circ} 43^{\prime} 52^{\prime \prime}$
\end{tabular}

Die beiden ersten Krystalle erläutern die einzige Regelmässigkeit, welche in diesen Schwankungen bemerkt werden kann; sie zeigen nämlich eine Abweichung einer der drei Rhomboëderflächen in einer senkrechten Ebene, entweder nach dem Ende des Krystalls hin oder von demselben weg.

Ein anderes Beispiel von geringerer Regelmässigkeit liefert ein Krystall von Chañarcillo, welcher folgende Messungen ergab:

$$
\begin{array}{lll}
r_{1} r_{2}=72^{0} 1^{\prime} 57^{\prime \prime} & e_{1} e_{2}=42^{\prime \prime 4} 44^{\prime} 57^{\prime \prime} \\
r_{2} r_{3}=72 \quad 1050 & e_{2} e_{3}=424653 \\
r_{3} r_{1}=72 \quad 520 & e_{3} e_{1}=424620
\end{array}
$$

Hier ist $e_{1}$ die Fläche, welche die Kante $r_{2} r_{3}$ abstumpft und so weiter in cyklischer Folge.

Es braucht wohl kaum bemerkt zu werden, dass die beobachteten Secunden nur das arithmetische Mittel von mehreren Beobachtungen sind. Die Ablesungen wurden geschätzt auf 10 Secunden und der Beobachtungsfehler kann nicht grösser sein als 20 Secunden.

Diese Beispiele zeigen die Nothwendigkeit, die Parameter einzig aus den allersorgfältigst ausgewählten Messungen abzuleiten. 


\section{$\S$ \%. Festgestellte Formen.}

In nachfolgender Tabelle sind die mit genügender Sicherheit festgestellten Formen zusammengestellt. Die Buchstaben der ersten Columne sind dieselben, wie ich sie in einer früher publicirten Liste im Min. Magaz. 1887, 7, 149 gegeben habe. Die letzte Columne enthält die frühern Beobachter der Formen und beziehen sich die angegebenen Namen auf folgende Schriften :

Ha üy , Traité de Minéralogie. 1822.

Lévy, Description d'une Collection, etc. 1837.

Mohs, Anfangsgründe der Naturgeschichte. 1839.

Hau smann, Handbuch der Mineralogie. 1847.

Miller, Phillips' Mineralogy. 1856.

Dufrénoy, Traité de Minéralogie. 1856.

Sella, Quadro delle forme etc. 1856.

Klein; Krystallberechnung. 1876.

Groth, Mineraliensammlung. 1878.

Streng, Neues Jahrbuch, 1878, S. 900. Ref. in dieser Zeitschr. 4, 321.

Schuster, diese Zeitschr. 1887, 12, 117.

t bedeutet, dass die betreffende Form auch vom Verf. beobachtet wurde. Diejenigen Gestalten, welchen kein Name beigesetzt ist, werden von allen Autoren angegeben. Die mit einem Fragezeichen versehenen Formen endlich bedürfen noch der weitern Bestätigung, ehe dieselben als absolut sicher angenommen werden können.

Die in $\oint 3$ angegebenen Formen sind die einzigen, welche definitiv aufgestellt werden konnten mit Bezug auf das Mineral (Pyrargyrit oder Proustit) und welche allein mit Sicherheit zu einer Discussion des Zusammenhangs und der Vertheilung der Flächen gebraucht werden können. Diejenigen dieser Formen, welche als unabhängige typische Formen auftreten und augenscheinlich nicht durch Zonen inducirt sind, werden in $\S 14$ besonders zusammengestellt werden.

Nach den Prismen und Rhomboëdern, sowie den Formen der Hauptzone are folgt die Anordnung der Formen in absteigender. Ordnung des Bruches $\frac{k}{-l}$, wo $\{h k l\}$ (nach Mille r) das Symbol der Form ist. Jede Gruppe von Formen, für welche dieser Bruch den gleichen Werth besitzt, ist eingeklammert und stellt eine Zone dar, welche von der Fläche $\{10 \pi 1\} R$ ausstrahlt. Innerbalb einer solchen Gruppe sind die Formen angeordnet in absteigender Folge des Bruches $\frac{h}{-l}$, d. i. in der Folge der Zunahme des Abstandes von der Basis (vergl. $§ 20)$. 


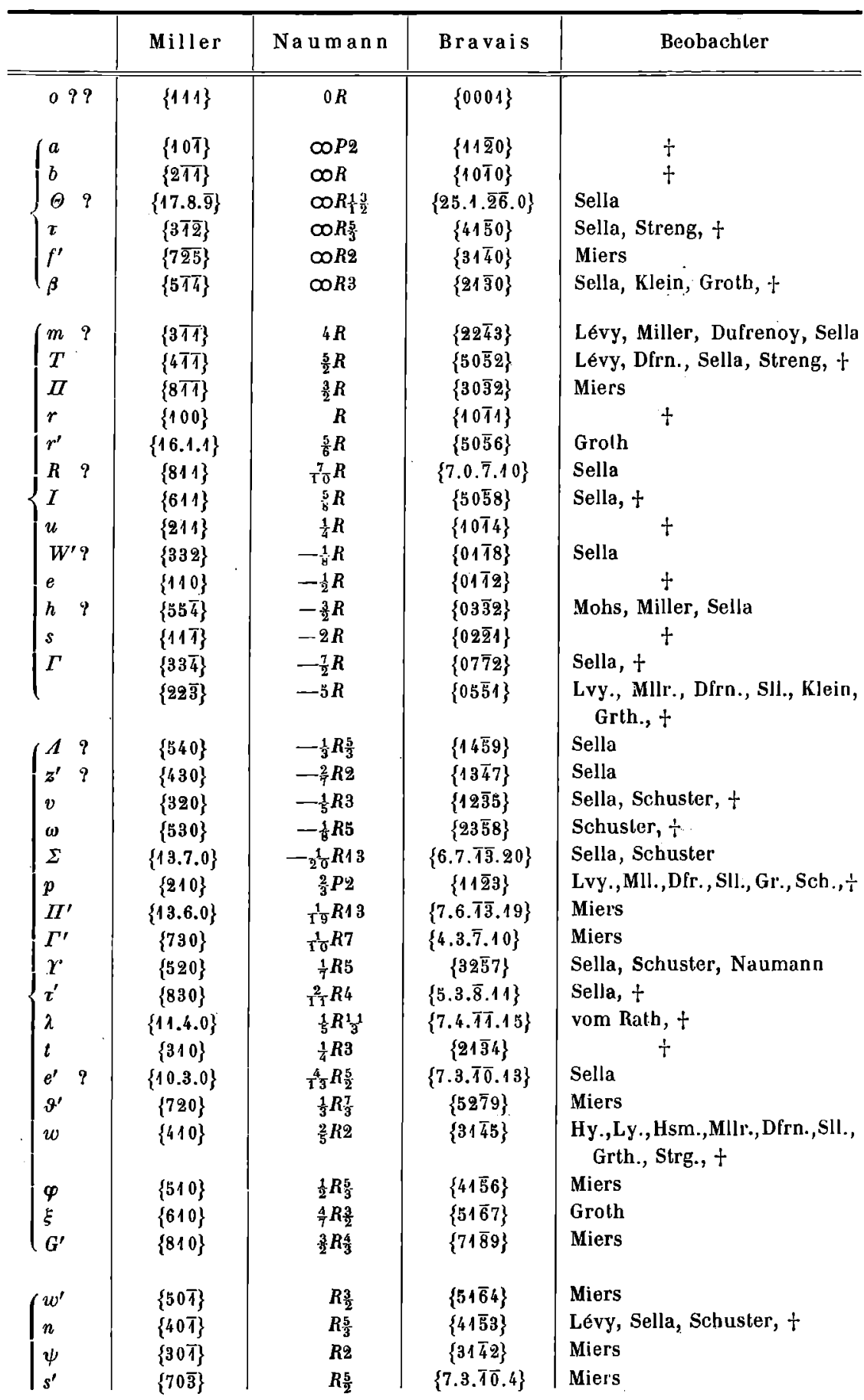


H. A. Miers.

\begin{tabular}{|c|c|c|c|c|}
\hline & Miller & Naumann & Bravais & Beobachter \\
\hline$v$ & $\{20 \bar{T}\}$ & $R 3$ & $\{21 \overline{3} 1\}$ & $t$ \\
\hline$\zeta$ & $\{90 \overline{5}\}$ & $R_{\frac{7}{2}}^{7}$ & $\{9.5 . \overline{4}, 4\}$ & Sella, Schuster, + \\
\hline$\gamma$ & $\{50 \overline{3}\}$ & $R 4$ & $\{53 \overline{8} 2\}$ & Sella, Groth, Strg., Schstr., $t$ \\
\hline$I^{\prime}$ & $\{17,0 . \overline{11}\}$ & $R_{\frac{14}{3}}$ & $\{17,11.2 \overline{8}, 6\}$ & Schuster \\
\hline$\{y$ & $\{30 \overline{2}\}$ & $R \tilde{s}$ & $\{32 \overline{5} 1\}$ & $\begin{array}{l}\text { Lvy., Mhs., Mllr., Dfrn., Sll. } \\
\text { Schst., }+\end{array}$ \\
\hline$\Delta$ & $\{19.0 . \overline{1} \overline{3}\}$ & $R_{3}^{16}$ & $\{19.13 . \overline{3} \overline{2} .6\}$ & Streng, Schuster, + \\
\hline$\Omega ?$ & $\{10.0 . \overline{7}\}$ & $R \frac{17}{3}$ & $\{10.7, \overline{1} \overline{7} .3\}$ & Sella \\
\hline$x ?$ & $\{70 \overline{5}\}$ & $R 6$ & $\{7.5 . \overline{2} \overline{2} .2\}$ & Sella \\
\hline & $\{40 \overline{3}\}$ & R7 & $\{43 \overline{7} 1\}$ & Hy.,Ly., Hsm., Dir., Sll., Sch., \\
\hline$\Delta \Delta^{\prime}$ & $\{17.0, \overline{1} \overline{3}\}$ & $R \frac{15}{2}$ & $\{17.13 .3 .40\}$ & Miers \\
\hline$\pi^{\prime}$ & $\{90 \overline{7}\}$ & $R 8$ & $\{9.7 . \overline{1} \overline{6} .2\}$ & Hausmann, Schuster \\
\hline$Z$ & $\{50 \overline{4}\}$ & $R 9$ & $\{54 \overline{9} 1\}$ & Miers \\
\hline$N^{\prime}$ & $\{17.0 . \overline{1} \overline{5}\}$ & $R 16$ & $\{17.15 . \overline{3} \overline{2} .2\}$ & Miers \\
\hline $\int \begin{array}{ll}c^{\prime} & ?\end{array}$ & $\{15.2 .1\}$ & $\frac{5}{8} R \frac{8}{5}$ & $\{13.3 . \overline{16} .16\}$ & Sella \\
\hline$\chi \quad ?$ & $\{82 \pi\}$ & $\frac{1}{3} R 3$ & $\{21 \overline{3} 3\}$ & Sella \\
\hline$a^{\prime}$ & $\{72 \pi\}$ & $\frac{1}{4} R 4$ & $\{\tilde{3} 3 \overline{8} 8\}$ & Sella, $t$ \\
\hline$\Psi$ & $\{621\}$ & $\frac{1}{7} R 7$ & $\{43 \overline{7} 7\}$ & Miers \\
\hline$\delta$ & $\left\{32^{\top}\right\}$ & $-\frac{1}{2} R 2$ & $\{13 \overline{4} 4\}$ & Sella, Klein, Groth, + \\
\hline$\mu^{\prime} ?$ & $\{85 \overline{3}\}$ & $-\frac{1}{2} R_{\frac{11}{5}}^{11}$ & $\{3.8 . \bar{T} .10\}$ & Sella \\
\hline$\varepsilon$ & $\{20.11 . \overline{7}\}$ & $-\frac{3}{8} R 3$ & $\{36 \overline{9} 8\}$ & Klein, Groth \\
\hline$M$ & $\{63 \overline{2}\}$ & $-\frac{2}{7} R 4$ & $\{35 \overline{8} 7\}$ & Hy., Sella, Streng, + \\
\hline$\eta^{\prime} ?$ & $\{84 \overline{3}\}$ & $-\frac{1}{3} R^{11}$ & $\{4.7 . \overline{1} .9\}$ & Sella \\
\hline$\{g$ & $\{64 \overline{3}\}$ & $-\frac{5}{7} R R^{\circ}$ & $\{27 \overline{9} 7\}$ & Miers \\
\hline$B$ & $\{17,5 . \overline{4}\}$ & $\frac{1}{6} R 7$ & $\{43 \overline{7} 6\}$ & Miers \\
\hline $\boldsymbol{K}$ & $\{21.6 . \overline{5}\}$ & $\frac{2}{1}_{1}^{2} R_{2}^{13}$ & $\{15.11 . \overline{2} \overline{6} .22\}$ & Miers \\
\hline$i^{i}$ & $\{51 \bar{T}\}$ & $\frac{2}{5} R 3$ & $\{42 \overline{6} 5\}$ & Miller, Sella \\
\hline$\sigma$ & $\{41 \bar{T}\}$ & $\frac{1}{4} R 5$ & $\{3254\}$ & Sella, $t$ \\
\hline$\left\{\Sigma^{\prime} ?\right.$ & $\{52 \overline{2}\}$ & $-\frac{1}{5} R 7$ & $\{34 \overline{7} 5\}$ & Hausmann, + \\
\hline la & $\{21 \pi\}$ & $-\frac{1}{2} R 3$ & $\{12 \overline{3} 2\}$ & $\begin{array}{l}\text { Ly., Mhs., Mllr., Dfrn., Sll., Kl. } \\
\text { Grth., }+\end{array}$ \\
\hline$\pi$ & $\{19.11 . \overline{1} \overline{2}\}$ & $-\frac{5}{6} R \frac{3}{1} \frac{1}{5}$ & $\{8.23 . \overline{3} T .18\}$ & Miers \\
\hline$\left\{\begin{array}{l}\Xi \\
s\end{array}\right.$ & $\begin{array}{c}\{27.6 . \overline{\overline{7}}\} \\
\{86 \overline{7}\}\end{array}$ & $\begin{array}{r}\frac{4}{13} R \frac{17}{4} \\
-\frac{11}{7} R \frac{15}{11}\end{array}$ & $\begin{array}{c}\{21.13 . \overline{34} .26\} \\
\{2.13 . \overline{1} \overline{5} .7\}\end{array}$ & $\begin{array}{l}\text { Miers } \\
\text { Miers }\end{array}$ \\
\hline$\vartheta$ & $\{97 \overline{8}\}$ & $-\frac{13}{8} R_{17} \frac{7}{3}$ & $\{2.15, \overline{1} \overline{7}, 8\}$ & Miers \\
\hline$Z^{\prime}$ & $\{13.9 . \overline{1}\}$ & $-\frac{16}{11} R \frac{3}{2}$ & $\{4.20 . \overline{2} \overline{4} .11\}$ & Miers \\
\hline$L$ & $\{19.4 . \overline{5}\}$ & $\begin{array}{r}11 \\
\frac{1}{3} R 4\end{array}$ & $\{53 \overline{8} 6\}$ & Miers \\
\hline$\eta$ & $\{28.13 . \overline{1} \overline{7}\}$ & $-{ }_{5}^{5} R 3$ & $\{5.10 . \overline{1} \overline{3} .8\}$ & Klein, Groth \\
\hline$A$ & $\{15.3 . \overline{4}\}$ & $\frac{5}{14} R \frac{19}{5}$ & $\{12.7 . \overline{9} .14\}$ & Sella, $t$ \\
\hline$x^{\prime}$ & $\{26.5 . \overline{7}\}$ & $\frac{3}{8} R \frac{11}{3}$ & $\{7.4 . \overline{1 \pi} .8\}$ & Miers \\
\hline$\Phi$ & $\{13.2 . \overline{3}\}$ & $\frac{1}{2} R_{3}^{8}$ & $\{11.5 . \overline{6} \overline{6} .12\}$ & Miers \\
\hline$W$ & $\{92 \overline{3}\}$ & $\frac{1}{4} R 6$ & $\{7.5 . \overline{1} \overline{2} .8\}$ & Rethwisch \\
\hline$m^{\prime}$ & $\{82 \overline{3}\}$ & $\frac{1}{7} R 11$ & $\{6.5 . \overline{11} .7\}$ & Miers \\
\hline
\end{tabular}




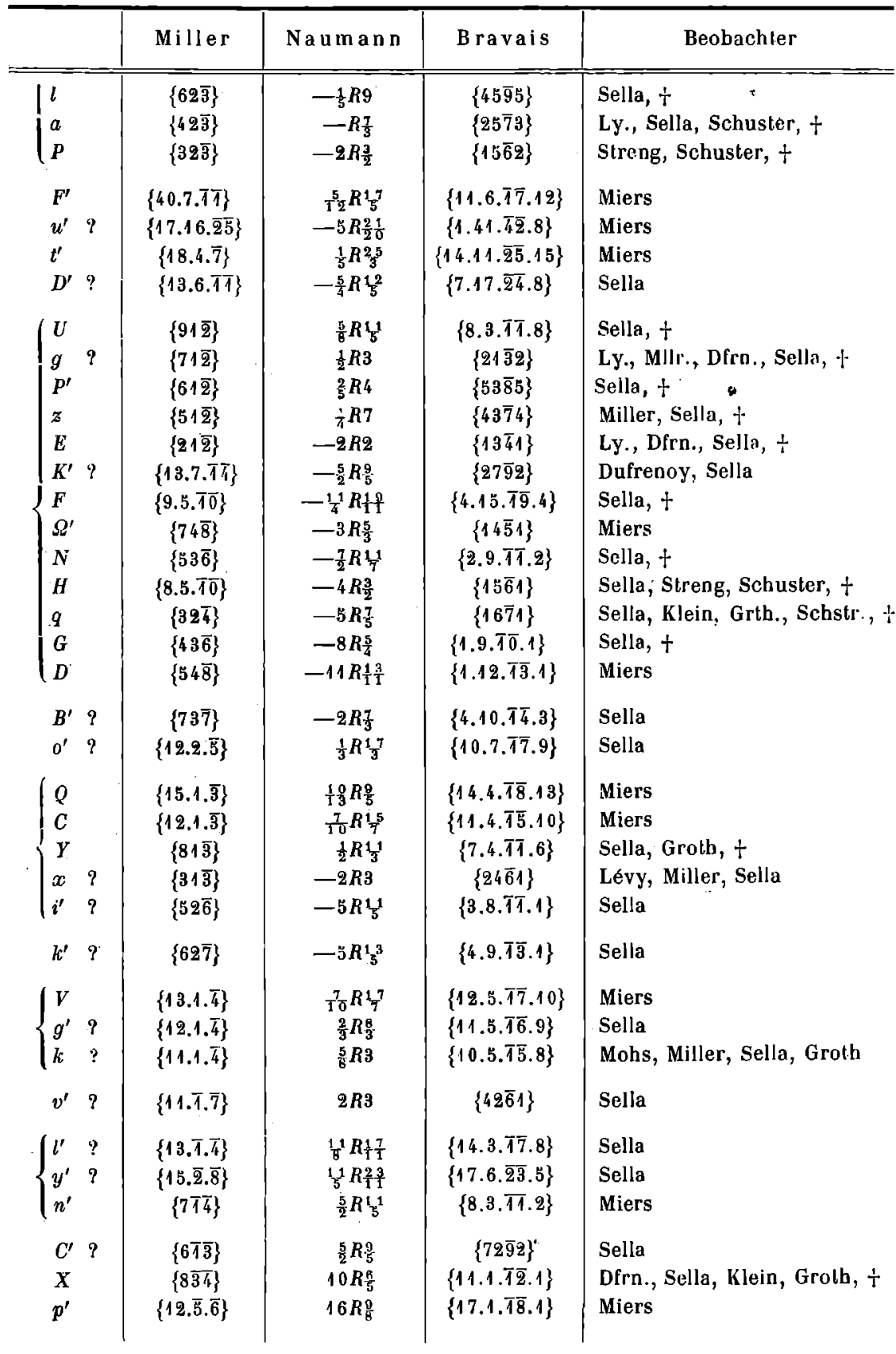




\section{\$ 8. Aussonderung ungewisser Formen.}

Eine grosse Anzahl (29) von den 111 von Rethw isch gegebenen Formen sind auszumustern. Für allgemeine Schlussfolgerungen in Bezug auf Vorkommen und relative Häufigkeit von gewissen Formen ist es von der grössten Bedeutung, dass nur solche in Betracht gezogen werden, welche gänzlich einwurfsfrei bestimmt sind. Bei nachstehender Beurtheilung waren hauptsächlich vier Erwägungen massgebend:

1) Eine Form ist noch nicht als fesigestellt zu betrachten, einzig weil sie von einer Anzahl Autoren aufgefuhrt wird, sobald Grunde zu der Vermuthung vorliegen, dass die Autoren von einander copirten.

2) Formên mit einfachen Indices sind nur dieses Umstandes wegen noch nicht zu acceptiren, da ganz naturlicherweise fruhere Beobachter zweifelhafte Formen zu solchen mit einfachen Indices rechneten.

3) Flächen in gestreiften Zonen, oder Zonen mit Vicinalflächen müssen ganz besonders sorgfältig kritisirt werden, weil dieselben oft mit einander verwechselt wurden.

4) Die Beobachtungen einiger Autoren sind unzuverlässig.

In Uebereinstimmung mit Erwägung (1) sind eine Anzahl Formen auszuscheiden als von Lé vy übernommen. Für (2) bieten die Formen $h, k, m$, $x$ (in vorstehender Tabelle durch ? gekennzeichnet) und $A^{\prime}$ Beispiele solcher, welche an den Krystallen des British Museum nicht aufzufinden waren und welche schwerlich von jenen frühern Autoren, die nur gewöhnlich vorkommende Formen erwähnen, beobachtet sein können. Aus (3) folgt, dass man nicht (wie Retbwisch gethan) Indices aus den Messungen Pbillips' berechnen darf, weil Derselbe, wie gezeigt werden wird, mehrfach Flächen $i_{n}$ gestreiften Zonen verwechselte.

(4) Was andere Autoren anbelangt, so hat bekanntlich Lév y eine sehr grosse Anzahl Figuren in seiner Beschreibung der Turner'schen Sammlung mitgetheilt. Beim Vergleich der Originalstulcke, welche jetzt einen Theil der in der Royal School of Mines befindlichen L ud la m'schen Sammlung bilden, mit der Lévy'schen Beschreibung ergab sich jedoch, dass die letztere sehr ungenau ist und dass die Figuren sehr oft nur wenig den Krystallen gleichen*). Es wurden deshalb alle, auf Lévy's Autorität beruhende Formen ausgeschieden.

Die ausgeschiedenen Formen und die Gründe ihrer Ausscheidung sind in der folgenden Tabelle zusammengestellt. Die Buchstaben sind dieselben

*) Herr T. Davies, welcher Gelegenheil hatte, zusammen mil dem verstorbenen Lu d l a m zahlreiche Exemplare dieser Sammlung mit Lévy's Beschreibung zu vergleichen, theilte mir mit, dass Letzterer auch bei anderen Mineralien, besonders Topas und Diamant, recht ungenau befunden wurde. 
wie die in der Min. Magaz. 7, S. 149 mitgetheilten Liste, in der dort beigegebenen Taf. IV kann die Lage dieser Fläche gefunden werden.

$b^{\prime}\left\{10 T_{2}\right\}_{2} R, \quad$ von Lév y angegeben.

$a^{\prime}\{30 \overline{3} 4\} \frac{3}{4} R, \quad$ berechnet von $R$ eth wisch aus $P$ billips.

$m\{40 \overline{4} 1\} 4 R, \quad$ von Lév y angegeben.

$X^{\prime}\{01 T 5\}-\frac{1}{5} R$,

$\zeta^{\prime}\{02 \overline{2} 5\}-\frac{2}{5} R, \quad$ berechnet von Rethwisch aus Hauy (s. unten).

$Y^{\prime}\{01 T 1\}-R, \quad$ von $L$ é v y angegeben.

$d^{\prime}\{0.14 . \overline{4} \overline{4} .1\}-14 R$,

$A^{\prime}\{22 \overline{4} 3\} \frac{4}{3} P 2, \quad-H$ aùy -

$\lambda^{\prime}\{11.11 . \overline{2} \overline{2} .8\}_{\frac{1}{4}}^{1} P 2,-H$ ausman $\mathrm{n}$, identisch mit der vorhergehenden. $\mu\{3255\} \frac{1}{5} R 5, \quad$ berechnet von $R$ et $\mathrm{bwisch}$ aus $\mathrm{Ha}$ a $\mathrm{y}$. (s. unten). $\nu\{4375\} \frac{1}{5} R \%, \quad$ irrigerw. v. Rethwis ch als v. Hau s mann angegeb. $\left.\nu^{\prime}\{15.5 . \overline{2} \overline{0} 32\}\right\}_{16}^{5} R 2$, berechnet von $R$ ethwisch aus $H$ a üy.

$q^{\prime}\{4.8 .1 \overline{2} .5\}-\frac{4}{5} R 3$, von Lévy angegeben.

$\psi^{\prime}\{11 \overline{2} 1\} 2 P 2, \quad-$ de Selle -

$\chi^{\prime}\{4.6 . T \overline{0} .7\}-\frac{2}{7} R 5,-\quad \quad-$

$\omega^{\prime}\{4.14 .18 .5\}-2 R \frac{9}{5},-\quad \ldots$

$\beta^{\prime}\{50 \overline{5} 1\} 5 R, \quad-\mathrm{Zippe}$

$\gamma^{\prime}\{80 \overline{8} 1\} 8 R$,

$\varepsilon^{\prime}\{0.5 . \overline{5} .16\}-\frac{5}{16} R, \quad-$

$E^{\prime}\{05 \overline{5} 4\}-\frac{5}{4} R$

$\xi^{\prime}\{8.4 . \overline{1} \overline{2} .5\} \frac{4}{5} R 3$

$\varrho^{\prime}\{31 \overline{4} 1\} 2 R 2$,

$\varphi^{\prime}\{4.12 . \overline{1} \overline{6} .1\}-8 R 2,-$

$\delta^{\prime}\{16.0 . \overline{1} \overline{6} .1\} 16 R, \quad$ Frenzel

$\sigma^{\prime}\{10.5 . \overline{1} \overline{5} .2\} \frac{5}{2} R 3, \quad-\quad \quad-$

$v^{\prime}\{5.15 . \overline{2} \overline{0} .2\}-5 R 2,-$

$x\{24 \overline{6} 1\}-2 R 3, \quad-\quad$ Mohs

$k\{10.5 . \overline{5} .8\} \frac{5}{8} R 3, \quad-\quad-\quad$ - (und bei Grolh)

$h\{03 \overline{3} 2\}-\frac{3}{2} R, \quad-$ Naumann -

$\{9.33 . \overline{4} \overline{2} .8\}-3 R \frac{7}{4},-\quad-$

Purgold (l. c.) fugt noch $-\frac{1}{4} R$ und $-4 R$ als unsichere Formen hinzu.

\section{\$ 9. Kritik der Messungen früherer Autoren.}

Phillips. - Durch die Güte des Herrn Prof. Le w is in Cambridge bin ich in den Stand gesetzt worden, ein Stück aus der jelzt einen Theil der dortigen Universitätssammlung bildenden Brooke'schen Gollection zu untersuchen, welches entweder eines der von $\mathrm{Ph}$ illips untersuchten Stücke, oder doch wenigstens eines von genau gleichem Habilus ist und wahrschein- 
lich von der Grube Abendröthe bei Andreasberg stammt. Ich konnte folgende unter den von Phillips gegebenen Formen bestäligen:

\begin{tabular}{|c|c|c|c|}
\hline Pbillips: & \multirow{2}{*}{$\begin{array}{c}\text { Symbol: } \\
\Gamma\{07 \overline{7} 2\}-\frac{7}{2} R\end{array}$} & Gemessen : & Berechnet \\
\hline$g$ & & $r \Gamma=57^{0} \quad 3^{\prime}$ & $570 \quad 9^{\prime}$ \\
\hline $\boldsymbol{P}$ & $r\{10 \bar{T} 1\}+R$ & $r r=7150$ & 7122 \\
\hline$l_{1}$ & $L\{53 \overline{8} 6\} \frac{1}{3} R 4$ & $L L=31 \quad 19$ & 3122 \\
\hline & & $L V=818$ & 818 \\
\hline$l_{2}$ & $x^{\prime}\{7.4 . \bar{T} .8\} \frac{3}{8} R^{11}$ & $V x^{\prime}=77$ & 713 \\
\hline$l_{3}$ & $V\{12.5 . \overline{17} .10\} \frac{7}{10} R^{17}$ & $V V=26 \quad 46$ & 2647 \\
\hline & & $r V=17 \quad 0$ & 1659 \\
\hline$i_{4}$ & $y\{32 \overline{5} 1\} R 5$ & $y y=4531$ & 4520 \\
\hline$l_{5}$ & $X\{11.1 . \overline{2} .1\} 10 R \frac{6}{5}$ & $X X=825$ & 834 \\
\hline 0 & $a\{1 \mid \overline{2} 0\} \infty P 2$ & - & - \\
\hline
\end{tabular}

Die Flächen $b, i_{2}, i, d_{5}$ der $\mathrm{Ph}$ ill jps'schen Figur sind zu matt, um genaue Messungen zu liefern; $i_{3}$ besteht aus vier, in der Zone $r \boldsymbol{Y} t$ in der Nachbarschaft von $Y$ gelegenen Flächen, welche weiter unten besprochen werden $(\$ 19)$.

Die Flächen in der Zone $P P(=r r)$ sind: $p\{11 \overline{2} 3\}, t\{21 \overline{3} 4\}, w\{31 \overline{4} 5\}$, $\varphi\{41 \overline{5} 6\}$. Zwischen $l_{3}$ und $l_{2}$ liegt eine Fläche von $F^{\prime}\{11.6 . \overline{7} .12\} \frac{5}{12} R 15$, bestimmt durch die Messung $V: F^{\prime}=6^{0} 19^{\prime}$ (berechnet 6011'). Die Messungen fur $l_{4}$ sind $: l_{4}: l_{4}=38^{\circ} 34$ und $r: l_{4}=40^{\circ} 7^{\prime}$.

Lév y. - Wie oben dargethan, sind dieses Autors Angaben nicht in dem Grade genau, um irgend etwas zu begründen, was nicht durch andere Beobachter bestätigl wird.

Mohs. $-k\{10.5 . \overline{5} .8\} \frac{5}{8} R 3$ ist zweifelhaft, da sie die zu $\eta\{5.10 . \overline{5} 5.8\}$ - $\frac{5}{8} R 3$ gehörige Form ist; ferner $x\{24 \overline{6} 1\}-2 R 3$, weil sie die zu $v^{\prime}\{42 \overline{6} 1\}$ $2 R 3$ gebörige Form ist. $k$ wird noch weiler unten zur Sprache kommen $(\S 15)$.

H a u sman n benutzt dieselben Buchstaben wie $\mathrm{Haüy}$, und es scheint kaum zweifelhaft, dass er dessen Verzeichniss der Formen copirt und nur eine neue, nämlich $K G \frac{1}{8}\{9.7 . \overline{1} \overline{6} .2\} R 8$, hinzugefugt hal. Alsdann ist sein $B C_{\overline{1}^{4}}(r)$ $=\{11.11 . \overline{2} \overline{2} .8)_{\pi}^{11} \mathrm{P} 2$ identisch mit $\mathrm{Ha}$ ä̈ y's $\frac{5}{3} E \frac{5}{3} D^{3} B^{5}(r)=\{44 \overline{8} 3\} \frac{8}{3} P 2$, welche beiden Formen ja fast zusammenfallen, und $\mathrm{H}$ a u s man n's $F A+G K \frac{5}{2}$ $(x)=\{34 \overline{7} 5\}-\frac{1}{5} R 7$ (nicht $\{43 \overline{7} 5\}+\frac{1}{5} R 7$, wie Rethw isch schreibt) identisch mil H a li y's ${ }^{1} E^{1} B^{3} D^{2}(x)=\{35 \overline{8} 7\}-\frac{2}{7} R 4$.

Naumann. - Die Form $h\{03 \overline{3} 2\}-\frac{3}{2} R$ wird von Naumann*) am Proustit angegeben; die betreffende Abbildung zeigt indessen eine auffallende Aehnlichkeit mit dem gewöhnlichen Habilus des Proustit (besonders von Freiberg), an welchem eine oscillatoriscbe Combination von $s\{02 \overline{2} 1\}-2 R$

*) Lehrb. d. Mineralogie, 1828, 602, Fig. 181. 
und $e\{01 T 2\}-\frac{1}{2} R$ leicht mil der Form $h$ verwechselt werden kann. Da die Angaben für diese Form nicht ganz scharfe sind, so wurde sie oben als zweifelhaft aufgefuhrt und mit ? versehen.

$\left.\{9.33 . \overline{4} \overline{2} .8\}-3 R_{\frac{7}{4}}^{*}\right)$ ist als unwahrscheinlich und von anderen Autoren nicht bestäligt ausgeschieden worden; Goldschmidt nimmt an, dass sie der Form $-3 R^{16}$ entspreche, doch kann kaum zweifelhaft sein, dass sie der Form $F=\{4.15 . \overline{1} .4\}-\frac{11}{4} R \frac{1}{1} \frac{9}{1}$ entspricht, welche eine ganz gewöhnliche Fläche am Andreasberger Pyrargyrit ist.

Sella. - Alle aus Sella's Quadro stammenden Formen sind, soweit sie der Bestảtigung bedürfen, zurückgestellt. Manche derselben sind später nicht mehr beobachtet worden, auch ist nicht zu ersehen, welche davon am Pyrargyrit und welche am Proustit beobachtet wurden, und da die von Sella beabsichtigte Beschreibung dieser Mineralien niemals publicirt wurde, so sind seine Messungen und andere Delails nicht zugänglich. Es wurden deshalb nur jene Formen S ella's in der Tabelle des $\oint 4$ aufgenommen, welche späterhin eine Bestätigung erfuhren.

Zip pe und de Selle. - Die von diesen Autoren gegebenen Formen wurden deshalb ausgeschieden, weil sie augenscheinlich auf Druckfehlern und ungenauer Interpretation fruherer Autoren beruhen.

Frenzel. - Dessen Formen sind ungewiss aus Grunden, die schon Rethwisch entwickelte (diese Zeitsch. 12, 72 Anmerk.).

Rethwisch. - Die aus Philllips Messungen abgeleitete Form $a^{\prime}$ ist ungewiss, wie bei Discussion der Philips'schen Angaben gezeigt wurde. $\nu\{43 \overline{7} \breve{5}\} \frac{1}{5} R 7$ ist eine irrthümliche Umwandlung des Ha u s man n'schen Zeichens $F A \frac{1}{4} G K \frac{5}{2}$ und muss $-\frac{1}{5} R 7$ heissen.

Im Jahre 1792 leitete Ha ü ${ }^{* *}$ ) die Formen des Rothgiltigerzes vom Rhombendodekaëder ab, da ihm zur Zeit der rhomboëdrische Charakter des Minerals noch unbekannt war. Unter den Flächen, welche er aufführt, ist das Ikositetraëder $202\{211\}$, das Tetrakishexaëder $\infty 0.53530\}$ und das $\mathrm{He}-$ xakisoktaëder $50 \frac{5}{4}\{541\}$. Fur diese Formen giebt er die berechneten Winkel auf Secunden und die Symbole können daher leicht aus den Winkeln abgeleitet werden. Diese Beschreibung ist berichtigt in seinem Traité de Minéralogie von 1801, wo die Primitiv-Form richlig beschrieben wurde.

Obgleich hieraus hervorgeht, dass die von $\mathrm{ihm}$ im J. 1792 angegebenen Formen keine Gultigkeit behalten konnten, nahm Rethwisch trotzdem Ha uy's Winkel, berechnet daraus die denselben entsprechenden Rhomboëder und Skalenoëder, nämlich $\{02 \overline{2} 5\}-\frac{2}{5} R,\{32 \overline{5} 5\} \frac{1}{5} R 5,\{15.5 . \overline{2} \overline{0} .32\}$ $\frac{5}{16} R 2$ und belrachtet dieselben als sichere Formen. Es ist daher kaum über-

*) Lehrb. d. reinen und angew. Krystallogr. 1830, 2, 312, Fig. 718.

**) Journ. d'hist. natur. 18, 216. 
raschend, dass, wie Relbwisch selbst bemerkt, die letzte Form nirgends anders erwähnt wird.

Der von G. vom Rath*) gemessene und abgebildete Krystall von Andreasberg wurde von $R$ eth w isch neu gemessen. In Uebereinstimmung mit vom Rath's Originalbeschreibung befindet sich eine Fläche $\lambda\{7.4 .1 T .15\}$ $\frac{1}{5} R \frac{11}{3}$ zwischen $r\{10 T 1\} R$ und $e\{01 T 2\}-\frac{1}{2} R$. In einer Zone zwischen $\lambda$ und dem Prisma $a\{1 \mid \overline{2} 0\} \infty P 2$ liegen zwei Flächen, welchen vom $R$ ath die Symbole $\mu\{3255\} 1{ }_{5} R 5$ und $\nu\{4375\} \frac{1}{5} R 7$ beilegte; jedoch sind die gemessenen Winkel $\mu \mu=45^{\circ} 23^{\prime}$ und $26^{\circ} 49^{\prime}$ diejenigen des Skalenoëders $a^{\prime}\{53 \overline{8} 8\}_{4}^{1} R 4$ statt $\mu\{32 \overline{5} 5\}{ }_{5}^{1} R 5$.

Rethwisch nimmt daher an, dass die Zone

$$
\left\{\begin{array} { l } 
{ a \{ 1 1 \overline { 2 } 0 \} } \\
{ W \{ 7 . 5 . \overline { 1 } \overline { 2 } . 8 \} } \\
{ a ^ { \prime } \{ 5 3 \overline { 8 } 8 \} } \\
{ t \{ 2 1 \overline { 3 } 4 \} }
\end{array} \quad \left\{\begin{array}{l}
a\{11 \overline{2} 0\} \\
\nu\{43 \overline{7} 5\} \\
\mu\{32 \overline{5} 5\} \\
\lambda\{7.4 . \overline{1} 1.15\} .
\end{array}\right.\right.
$$

Da die Rethwisch'schen Angaben keine directe Entscheidung gestatten, hat Herr G. Seligmann, welchem der Krystall gehört, die Messung freundlichst wiederholt und gefunden :

Die Flächen zwischen $r e$ gehören der Form $\lambda$ und nicht $t$ an; die Neigung der Fläche zu $e$ wurde einige Male gemessen zu 18016'; $18^{0} 16 \frac{1}{2}$; $18^{0} 25 \frac{1}{2}^{\prime}$. Berechnel $\lambda e=18^{0} 32^{\prime}, t e=19^{\circ} 46^{\prime}$.

Das gelegentliche Auftreten von $t$ wird indessen angedeutet durch ene Messung, welche $19033 x^{\prime}$ ergab.

vom Rath's $\mu$ ist gleich $a^{\prime}=\{53 \overline{8} 8\} \frac{1}{4} R 4$.

$$
\text { Gemessen : } \mu: \mu=\frac{26^{0} 421^{\prime}}{4528-46^{0} 46 \frac{1}{2}^{\prime}} \quad \text { Berechnel: } a^{\prime}: a^{\prime}=26^{0} 46^{\prime}
$$

In der Zone $\lambda \mu \nu$ ergab sich :

$$
\begin{array}{ll}
\lambda: \mu=7031 \frac{1}{2}^{\prime}-41 \frac{1}{2}^{\prime} & \text { Berechnet }: t: a^{\prime}=70481^{\prime} \quad \lambda: a^{\prime}=7034 \frac{1}{2}^{\prime} \\
\mu: \nu=1130 \frac{1}{2}-49 & a^{\prime}: W=1132 \\
\nu: a=4017 \frac{1}{2}-24 & W: a=4022 .
\end{array}
$$

Es ist daher kein Zweifel mehr, dass $\lambda, a^{\prime}, W$ als wohlbegründete Formen aufzufubren sind.

Die Formen $0, m, h, x$ sind, obgleich in die Liste aufgenornmen, mit Fragezeichen versehen und zwar aus folgenden Gründen :

$o$ erscheint nie als glatte, sondern stets als drusige Fläche, gebildet aus Rbomboëderflächen, oder aber sie ist ganz raub.

$m$ scheint von Lé v y hergenommen zu sein (siehe oben).

$h$ ist von Na uma n n ubernommen (s. oben).

*) Pogg. Ann. 158, 4z2, Taf. V, Fig. 22. 
$x$ ist von Mohs entnommen, jedoch als zweifelhaft zu betrachten, da sie die inverse Form der von Sell a gegebenen $v^{\prime}\{42 \overline{6} 1\} 2 R 3(\mathrm{~s} . \$ 15)$ ist, und dürfte auch nicht auf eine unabhängige Aulorität gestủtzl sein.

\section{\$10. Messungen und Charakter der Flächen.}

Der Ausdruck »glatt « zeigt in der folgenden Beschreibung an, dass die beobachlete Fläche eine wirkliche, einen guten Reflex gebende Ebene gewesen ist.

$$
\begin{gathered}
a\{1 \mid \overline{2} 0\} . \\
b\{10 T 0\} . \\
c\{43 \overline{7} 1\} . \\
d\{12 \overline{3} 2\} . \\
e\{01 \overline{1} 2\} . \\
f\{05 \overline{5} 1\} . \\
g ?\{21 \overline{3} 2\} . \\
l\{45 \overline{9} 5\} \\
n\{41 \overline{5} 3\} . \\
o ?\{0001\} . \\
p\{11 \overline{2} 3\} .
\end{gathered}
$$$$
q\{16 \overline{7} 1\} .
$$$$
r\{10 T 1\} \text {. }
$$$$
s\{02 \overline{2} 1\} \text {. }
$$$$
t\{21 \overline{3} 4\} .
$$$$
\text { " }\{10 \overline{4}\} \text {. }
$$$$
v\{21 \overline{3} 1\} \text {. }
$$$$
w\{3 \mid \overline{4} 5\} \text {. }
$$$$
y\{32 \overline{5} 1\} .
$$$$
z\{43 \overline{7} 4\} \text {. }
$$$$
a^{\prime}\{53 \overline{8} 8\} \text {. }
$$$$
f^{\prime}\{31 \overline{4} 0\} \text {. }
$$$$
m^{\prime}\{6.5 . \overline{1} .7\} \text {. }
$$$$
n^{\prime}\{8.3 .1 \bar{T} .2\} \text {. }
$$$$
p^{\prime}\{17.1 . \overline{1} \overline{8} .1\} \text {. }
$$

Glatt; beim Pyrargyrit gestreift parallel $a v \alpha q$, beim Proustit parallel $\tau$.

Glatt; beim Pyrargyrit gestreift parallel $X$.

Schmal, unehen; $a: c=11015^{\prime}$ (berechnet $11015^{\prime}$ ).

Glatt, immer klein; am Pyrargyrit mit $f Y$.

Glatt, gestreift $\| e$.

Nicht ganz glatt; $f: a=32^{0} 10^{\prime}$ (herecbnet $32^{\circ} 14^{\prime}$ ).

Schmal, uneben $(\mathrm{s} . \$ \S 15,18)$.

Glänzend, klein; an der Kante $a p$ (Pyrargyrit) $a: l=$ $34^{055^{\prime}}$ (berechnet 35015').

Nicht ganz eben, nur am aufgewachsenen Ende, s. $\$ 11(6)$.

Stets drusig, nie als wabre Fläche.

Gewöhnlich uneben oder gekrümmt in der Zone $e r$, zuweilen vollkommen eben au Pyrargyril (vergl. Dufrénoy, S. 433).

Glatt, s. $§ 11(14)$.

Glatt, am Pyrargyrit parallel $e$ gestreift.

Glatt, gestreift \| $e$ am Proustit, beim Pyrargyrit zuweilen etwas uneben.

Glatt, öfters uneben in der Zone $e r$.

Uneben und rauh, die Kante ee abstumpfend. Einmal glatt beobachtet am Pyrargyrit von Joachimsthal.

Glatt, bei beiden Mineralien gestreift $\| a$.

Uneben am Pyrargyrit; $w: w=15^{0} 27^{\prime}$ (ber. 1501.0'), glatt am Proustit.

Glatt; beim Pyrargyrit zuweilen gross.

Glänzend, klein.

Uneben, gestreift in der Zone $r a^{\prime} . \quad a^{\prime} a^{\prime}=44^{0} 16^{\prime}$. (ber. $\left.45^{0} 22^{\prime}\right)$.

S. $\oint 11(1)$.

Glatt, s. $\S 11(13),(16)$.

Gestreift, s. $\ 11$, (4) Proustit.

Schmal, s. $\oint 11(11),(23)$. 


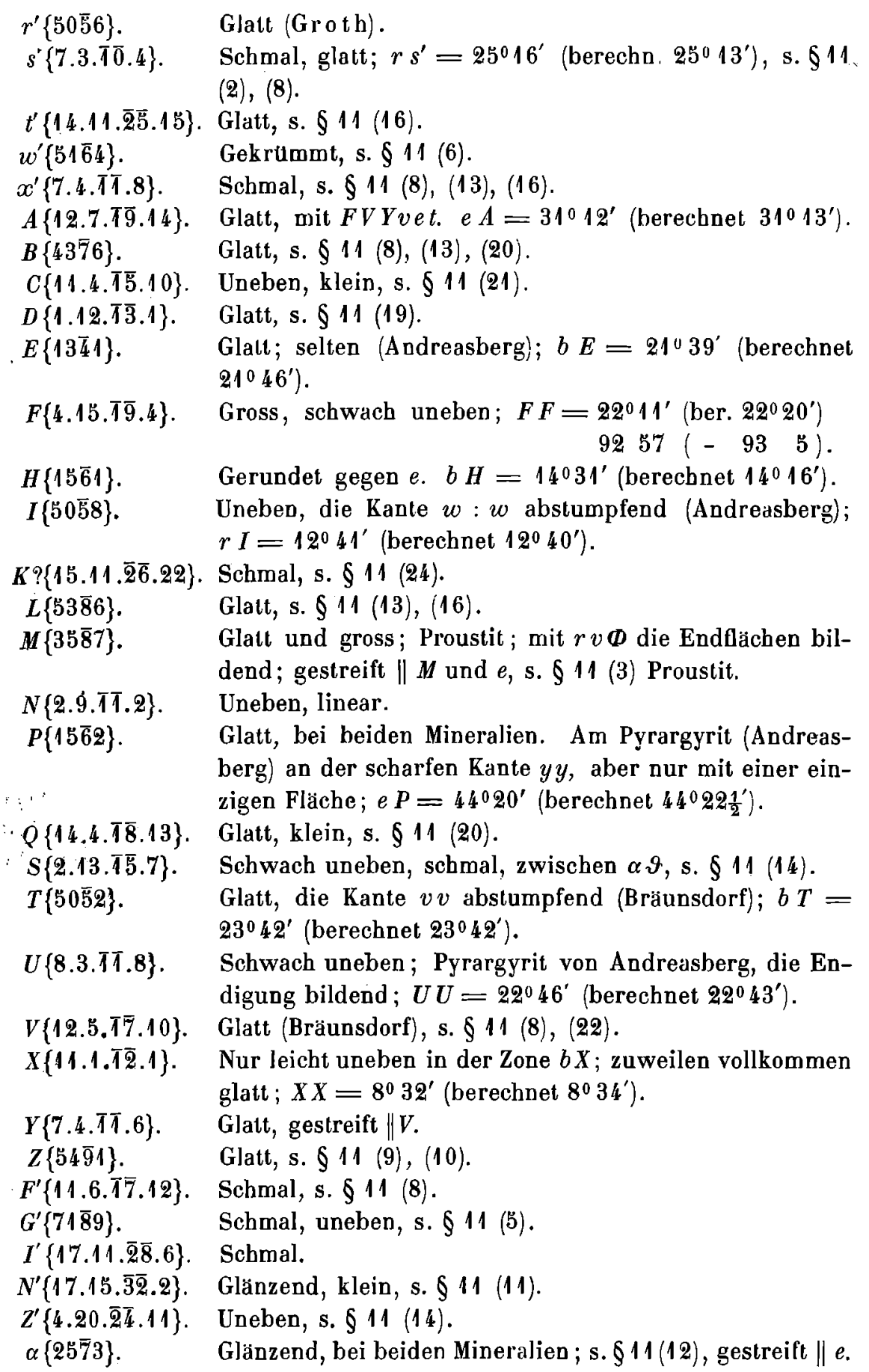


$\beta\{21 \overline{3} 0\} . \quad$ Glatt; Pyrargyrit; $a \beta=10^{\circ} 47^{\prime}$ (berechnet $10^{0} 53 \frac{1}{2}^{\prime}$ ).

$\gamma\{53 \overline{8} 2\}$.

Glänzend, gewöhnliche Fläche.

$\delta\{13 \overline{4} 4\}$.

Glatt.

$\zeta\{9.5 . \overline{4} .4\}$. Uneben, gekrummt in der Zone $a v$, zwischen $y v$; $a \zeta=220^{\prime}$ (berechnet $21^{\circ} 41^{\prime}$ ).

$\vartheta\{2.15 . \overline{1} \overline{7} .8\}$ Glatt, schmale Flächen zwischen $\alpha \alpha$, s. $\S 11$ (12), (14)

$\lambda\{7.4 .1 T .15\} . \quad$ Elwas uneben, in der Zone er, s. $\oint 9$.

$\xi\{51 \overline{6} 7\}$.

Gestreift.

$\pi\{8.23 . \overline{3} T .18\}$. Glatt, in oscillatorischer Combinalion mit $e \alpha, s . \S 11$ (12).

$\varrho\{27 \overline{9} 7\}$.

Glatt, in oscillatorischer Combination mil $e \alpha, \mathrm{s} . \S 11(12)$.

$\sigma\{3254\}$.

Schmal, s. $§ 11(8)$.

$\tau\{41 \overline{5} 0\}$.

Glatt, klein, s. $\oint 11$ (11).

$v\{12 \overline{3} 5\}$.

Schmal.

$\varphi\{4156\}$.

Glatt, aber klein, s. $§ 11(5),(8),(12),(20)$.

$\psi\{31 \overline{4} 2\}$.

Schmal, s. §11 (2), (12).

$\omega\{23 \overline{5} 8\}$.

Schmal.

$\vartheta^{\prime}\{5279\}$.

Glatt, s. $\oint 11$ (1) Proustit.

$\tau^{\prime}\{5.3 . \overline{8} .11\}$.

Schmal.

$n^{\prime}\{9.7 . \overline{1} \overline{6} .2\}$. Schmal.

$\Gamma\{07 \overline{7} 2\}$.

Leicht gekrümmt, gross, mit $F q$, etc.; $r \Gamma=57010^{\prime}$ (berechnet $57^{\circ} 9^{\prime}$ ).

$\Delta\{19.13 . \overline{3} \overline{2} .6\}$. Schmal.

$\Xi\{21.13 . \overline{34} .26\}$. Schmal, s. $\S 11(13)$.

$\Pi\{30 \overline{3} 2\}$. Leicht uneben, die Kante $n n$ abstumpfend, s. $§ 11$ (2).

$\Sigma\{6.7 . \overline{1}$. 20$\}$. Schmal.

$\Phi\{11.5 . \overline{1} \overline{6} .12\}$. Glänzend, aber uneben, s. $\S 11$ (3) Proustit; in $\{7.3 . \overline{1} \overline{0} .7\}$ ubbergehend.

$r\{32 \overline{5} 7\}$. Glatt, schmal, s. $\oint 11(13)$.

$\Gamma^{\prime}\{4.3 . \overline{7} .10\} . \quad$ Matt, s. $\oint 11(4)$.

$\Delta^{\prime}\{17.13 . \overline{3} \overline{0} .4\}$. Scbmal, s. $\S 11(9)$.

$\Pi^{\prime}\{7.6 . \overline{1} \overline{3} .19\} . \quad$ Schmal, s. $\oint 11(2)$.

$\Omega^{\prime}\{14 \overline{5} 1\}$. Schmal, s. $\$ 11(18)$.

\section{\$11. Messungen von neuen Formen.}

a) Sichere Formen.

Pyrargyrit.

(1). $f^{\prime}=\{31 \overline{4} 0\} \infty R 2$, Andreasberg. Zwei Flächen an einer Kante des Prismas $a\{11 \overline{2} 0\}$. In seiner Begleitung tritt $q\{16 \overline{7} 1\}$ auf, welches unter ihm an dem aufgewachsenen Ende des Krystalles erscheint.

$$
\begin{aligned}
a: f^{\prime}= & 16^{0} 10^{\prime}-16^{0} 43^{\prime} \text { erste Fläche. Berechnet } 16^{0} 6^{\prime} . \\
& 1614-17 \text { a weile - }
\end{aligned}
$$




$$
\text { (2) } \begin{array}{rlrlrl}
\Pi & =\{30 \overline{3} 2\} \frac{3}{2} R & r: \Pi & =1200^{\prime} & \text { Ber. } 11028 \frac{1}{2}^{\prime} \\
s^{\prime} & =\{7.3 . \overline{0} .4\} R_{\overline{2}}^{5} & a: s^{\prime} & =29 \quad 9 & -296 \\
\psi & =\{31 \overline{4} 2\} R 2 & \alpha: \psi & =3423 & -3450 \\
& \{23.9 . \overline{3} \overline{2} .14\} R_{\overline{7}}^{16} & a:(23.9 . \overline{3} \overline{2} .14)=31 & 2 & -3121
\end{array}
$$

Andreasberg; am aufgewachsenen Ende eines Krystalles mit $r n \gamma$; $s^{\prime} \psi$ schmale Flächen zwischen $\gamma n$.

(3) $\quad \Pi^{\prime}=\{7.6 . \overline{1} \overline{3} .19\}_{1 \overline{9}}^{1} R 13 \quad e: \Pi^{\prime}=14^{0} 37^{\prime}-14^{\circ} 59^{\prime}$ Berechn. 14.050'.

Andreasberg; beobachtet an sechs Krystallen, schmale Fläche.

(4) $\Gamma^{\prime}=\{4.3 . \overline{7} .10\} \frac{1}{10} R 7 \quad e: \Gamma^{\prime}=16^{0} 6^{\prime}$ erste Fläche Belechn. $16^{0} 2^{\prime}$. 1557 zweite -

Andreasberg; zwischen $e v$ als schmale, matle Flăche.

$$
\begin{aligned}
\varphi & =\{41 \overline{5} 6\}_{\frac{1}{2} R \frac{5}{3}} & r: \varphi=9058^{\prime} & \text { Berechnet } 10^{0} 5^{\prime} \\
G^{\prime} & =\{71 \overline{8} 9\}_{3}^{2} R \frac{4}{3} & r: G^{\prime}=620 & -
\end{aligned}
$$

Andreasberg; zwischen $r$ und $w$ in der Zone der Flächen $\dot{y} r G^{\prime} \varphi w t$. $G^{\prime}$ schmale Fläche.

Ueber $\varphi$ s. auch unter (8), (12), (20).

$$
w^{\prime}=\{51 \overline{6} 4\} R \frac{3}{2} \quad a: w^{\prime}=42012^{\prime} \quad \text { Berechnet } 42041^{\prime}
$$

Andreasberg; mit $r n q v G$ am aufgewachsenen Ende. $w^{\prime}$ ist gegen $n$ zu gekrummt und giebt einen Reflex bis:

$$
\begin{aligned}
& a: ?=48^{04} 4^{\prime} \\
& a: n=50 \quad 16
\end{aligned}
$$

(7) $\quad \psi=\{31 \overline{4} 2\} R 2$, s. unter (2) und (12).

$$
\begin{array}{llrl}
s^{\prime}=\{7.3 . \overline{1} \overline{0} .4\} R & r: s^{\prime}=25^{0} 16^{\prime} & \text { Berechnet } 25^{0} 13^{\prime} \\
\varphi=\{41 \overline{5} 6\}_{\frac{1}{2}} R \frac{5}{3} & r: \varphi=1018 & - & 10 \quad 5
\end{array}
$$

Andreasberg; $s^{\prime}$ als schmale glänzende Fläche zwischen $r v ; \varphi$ glänzende Fläche zwischen $r t$ in der $y \gamma v s^{\prime} r \varphi t$ enthaltenden Zone; mit $V F^{\prime} x^{\prime} \sigma B$. Ueber $s^{\prime}$ siehe auch unter (2).

$$
\begin{aligned}
& V=\{12.5 . \overline{1} \overline{7} .10\}_{10}^{7} R_{7}^{17} \\
& t: V=73^{0} 36^{\prime} \\
& \text { Berechnet } 73^{\circ} 50^{\prime} \\
& F^{\prime}=\{11.6 . \overline{1} \overline{7} .12\}_{\frac{5}{12}} R \frac{17}{5} \\
& t: F^{\prime}=6740 \\
& 6739 \\
& x^{\prime}=\{7.4 . T T .8\} \frac{3}{8} R \frac{1}{3} \\
& t: x^{\prime}=66 \quad 28 \\
& \begin{array}{ll}
66 & 37
\end{array} \\
& \sigma=\{32 \overline{5} 4\} \frac{1}{4} R 5 \\
& t: \sigma=6341 \\
& 63 \quad 16 \\
& B=\{43 \overline{7} 6\} \frac{1}{6} R 7 \\
& t: B=64 \quad 6 \\
& \begin{array}{ll}
60 & 49
\end{array}
\end{aligned}
$$

Betreffs V s. (22), uber $x^{\prime}$ s. (13) und uber $B$ s. (20).

$$
\begin{aligned}
\Delta^{\prime} & =\{17.13 . \overline{3} \overline{0} .4\} R^{1 \frac{5}{2}} \\
Z & =\{54 \overline{9} 1\} R 9
\end{aligned}
$$$$
y: \Delta^{\prime}=\mathbf{5}^{0} \mathbf{3}^{\prime}
$$$$
y: Z=636
$$
Berechnet $\mathbf{5}^{0} \mathbf{2}^{\prime}$ $6 \quad 46$

Freiberg; scbmale Flächen in der gestreiften, $y \mathcal{A} \Omega \Delta^{\prime} Z$ enthaltenden Zone, an einem Krystall mit der Endigung $Y d f$. 
(10) $Z=\{54 \overline{9} 1\} R 9$

$a: Z=9012^{\prime}$

Berechnet $9047^{\prime}$

Fundort unbekannt; $Z$ glänzende Fläche, mil avtyqe $\beta \tau \zeta$; in der Zone $q q$. Siehe auch unter $(9)$.

$$
\begin{array}{rlr}
N^{\prime}=\{17.15 . \overline{3} \overline{2} .2\} R 16 & a: N^{\prime}=4059 & \text { Berechnel } 4058^{\prime} \\
p^{\prime}=\{17.1 . \overline{1} \overline{8} .1\} 16 R \frac{9}{8} & b: p^{\prime}=434 & 434
\end{array}
$$

Andreasberg; $N^{\prime}$ glänzende Fläche zwischen $a c ; p^{\prime}$ schmale Fläche zwischen $b X$; mit $a b \tau p X q$ und zwei Flächen von $\{14.9 . \overline{2} \overline{3} .32\}$.

$$
\begin{aligned}
& a:(14.9 . \overline{2} \overline{3} .32)=72^{\circ} 30^{\prime} \quad \text { Berechnet } 72^{\circ} 33^{\prime}{ }^{\prime} \\
& 72 \quad 37 \\
& a: \tau \quad=1856-19^{0} 13^{\prime} \quad 196 \frac{1}{2}
\end{aligned}
$$

$$
\begin{aligned}
& \text { (12) } \\
& \varrho=\{27 \overline{9} 7\}-\frac{5}{7} R \frac{9}{5} \\
& e: \varrho=23^{\circ} 23^{\prime} \\
& \pi=\{8.23 . \overline{3} \overline{1} .18\}-{ }_{6}^{5} R_{15}^{3} \\
& e: \pi=3158 \\
& 3122 \\
& \vartheta=\{2.15 .17 .8\}-\frac{13}{8} R_{1}^{17} \\
& \vartheta: \vartheta=10 \text { 4.9 u. } 10^{\circ} 50^{\prime} \\
& 1050 \\
& \varphi=\{41 \overline{5} 6\} \frac{1}{2} R \frac{5}{3} \\
& e: \varphi=2535-2541 \\
& 2535 \\
& \psi=\{31 \overline{4} 2\} R 2 \\
& e: \psi=5441-5451 \\
& 5510
\end{aligned}
$$

Fundort unbekannt. Alles glänzende Flächen mit abert $\alpha g ; \varphi$ zwischen $e r ; \varrho$ und $\pi$ liegen zwischen $e \alpha ; \vartheta$ zwischen $\alpha \alpha$.

$$
\begin{aligned}
& \text { e : } \alpha=39^{\circ} \quad 0^{\prime} \quad \text { Berechnet } 39^{\circ} \quad 2^{\prime} \\
& \pi: \pi=2330 \quad 2324 \\
& b: B=52024^{\prime} \quad \text { Berechnet 520 } 9^{\prime} \\
& b: L=4724 \\
& 4726 \\
& b: x^{\prime}=4633 \\
& 46 \quad 24 \\
& m^{\prime}: m^{\prime}=41 \text { 21 } \\
& 4124 \\
& b: \xi=48 \quad 8 \\
& 48 \quad 7
\end{aligned}
$$$$
\text { (13) } B=\{43 \overline{7} 6\} \frac{1}{6} R 7
$$$$
L=\{53 \overline{8} 6\} \frac{1}{3} R 4
$$$$
x^{\prime}=\{7.4 . T T \cdot 8\} \frac{3}{8} R \frac{11}{3}
$$$$
m^{\prime}=\{6.5 . \overline{1} \text { T. } 7\}_{7}^{1} R 11
$$$$
\Xi=\{21.13 . \overline{3} \overline{4} \cdot 26\}_{\frac{4}{13}} H_{\frac{1}{4}}
$$

Andreasberg; mit $r y X a b t w \varphi G^{\prime} p r$, alles glänżende, obgleich schmale Flächen.

$$
\begin{aligned}
& r: r=18^{0} 19^{\prime} \quad \text { Berechnet } 18^{\circ} 34^{\prime} \\
& r: L=1553 \quad 1551 \\
& L: L=3127 \quad 3122 \\
& b: m^{\prime}=46 \quad 0 \quad 46 \quad 2 \frac{1}{2}
\end{aligned}
$$

(14) $\begin{array}{rlr}S=\{2.13 . \overline{5} .7\}-\frac{11}{7} R \frac{15}{1} & S: S=12023^{\prime} & \text { Berechnet } 12023^{\prime} \\ \vartheta=\{2.15 . \overline{1} \overline{7} .8\}-\frac{13}{8} R \frac{17}{1} \quad \vartheta: \vartheta=1052 & 1050\end{array}$

Grube Morgenstern, Freiberg; beides glänzende Flächen zwischen $\alpha \alpha$, mil $e q ; \vartheta$ auch in der Zone $e q$.

$$
\begin{array}{lrl}
e: \vartheta=3706^{\prime} & \text { Berechnet } 370 \mathbf{5}^{\prime}{ }^{\prime} \\
e: q=56 \quad 18 & 5615
\end{array}
$$




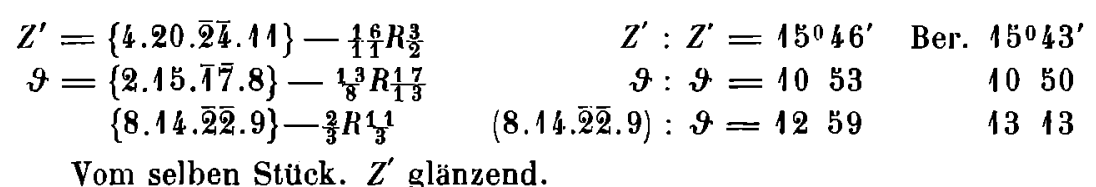

(15) $L x^{\prime}$ s. (8) (13).

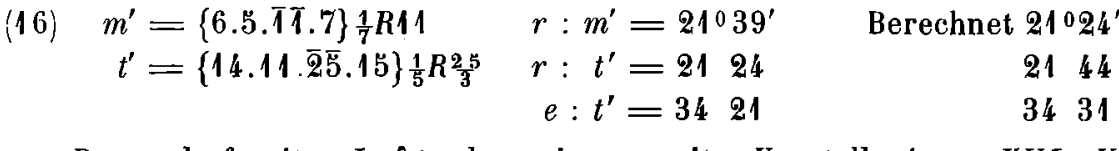

Bräunsdorf; mil erLp $L t w d$; an einemzweiten Krystall mil aryXVLxY.

$$
\begin{aligned}
& r: m^{\prime}=21^{0} 39^{\prime} \quad \text { Berechnel 21024' } \\
& r: m^{\prime}=64 \quad 5 \quad 64 \quad 9 \\
& r: L=1552 \quad 1551 \\
& r: L=64 \quad 9 \quad 64 \quad 8 \\
& L: x^{\prime}=110 \quad 15
\end{aligned}
$$

Siebe auch (13).

(17) $\quad F^{\prime}=\{11.6 . \overline{1} \cdot 12\}_{\frac{5}{12}} R \frac{17}{5}$. Siehe (8) und (9).

(18) $\Omega^{\prime}=\{14 \overline{51}\}-3 R_{\frac{5}{3}} \quad r: \Omega^{\prime}=52059^{\prime} \quad$ Berechnet $53^{0} 3 \frac{1}{2}^{\prime}$

Andreasberg; zwischen $F q$, schmale Fläche.

(19) $D=\{1.12 . \overline{1} 3.1\}-11 R_{11}^{13} \quad b: D=6^{0} 26^{\prime}$ Berechnet $6^{0} 24^{\prime}$ $\{2.5 .1 \overline{7} .2\}-\frac{13}{2} R_{\frac{1}{1}} \frac{1}{3} \quad b:(2.5 .1 \overline{7} .2)=9^{0} 57^{\prime} \quad 955$

Bräunsdorf. Mit eqabH einen Theil einer gekrümmten Zone $b q$ bildend. $\quad b: H=14^{0} 31^{\prime} \quad$ Berechnet 140 16'

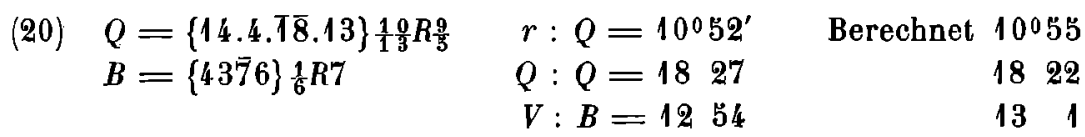

Andreasberg. Breite Fläche an der Kanle zwischen $r V$, mit $r t \varphi F v y$ $V L B X$ (Fig. 8).

$$
r: \varphi=10^{\circ} 7^{\prime} \quad \text { Berechnet } 100^{\prime}
$$

(21) $C=\{11.4 .75 .10\} \frac{7}{10} R{ }_{7}^{15} \quad r: C=13^{0} 47^{\prime} \quad$ Berechnet 13042' Andreasberg. Schmale glänzende Fläche zwischen $r \boldsymbol{Y}$.

$$
\begin{array}{rr}
r: Y=20^{\circ} 43^{\prime} & \text { Berechnet } 20^{\circ} 48^{\prime} \\
Y: Y=3434 & 3434
\end{array}
$$

(22) $V=\{12.5 . \overline{1} \overline{7} .10\} \frac{7}{10} R^{1.7} \quad \begin{array}{llr}V: \dot{V}=67038 & \text { Berechnet } 67034 \\ & V: V=26 \quad 50 & 26\end{array}$

Andreasberg. Mit y a $\omega p t w \varphi G^{\prime} x^{\prime} X m^{\prime} \Delta$ und $\{7.3 . \overline{1} \overline{0} .6\}$, letzteres eine matte Flïche zwischen $V x^{\prime}$.

$$
V:(7.3 . \overline{1} \overline{0} .6)=0^{0} 31^{\prime} \quad \text { Berechnet } 0^{0} 39^{\prime} \text {. S. a. }(8) \text {. }
$$


(23) $p^{\prime}=\{17.1 . \overline{8} 8.1\} 16 R \frac{9}{8} \quad b: p^{\prime}=4^{0} 25^{\prime}$ u. $4^{041^{\prime}}$ Berechnet $4^{0} 34^{\prime}$ $p^{\prime}: p^{\prime}=6 \quad 0$ 539

Andreasberg; glänzende Fläche zwischen $b X$. S. a. (11).

Proustit.

(1) $\vartheta^{\prime}=\{5279\} \frac{1}{2} R \frac{7}{3} \quad \vartheta^{\prime}: \vartheta^{\prime}=44^{0} 30^{\prime} \quad$ Berechnet $44^{\circ} 6^{\prime}$

Chili; an einem Krystallbruchstlick mit $v$, glänzende Flächen in der Zone $v v$.

(2) $\Psi=\{43 \overline{7} 7\} \frac{1}{7} R 7$

$$
\begin{array}{lr}
\Psi: \Psi=31010^{\prime} & \text { Berechnet } 3106^{\prime} \\
\Psi: \Psi=4154 & 4154
\end{array}
$$

Chañarcillo; glänzende Flächen mit ervwds Ma (Fig. '7).

$$
\Psi: \Psi=31^{\circ} 18^{\prime}
$$

Chañarcillo. Mit evar $w d M$; zwei Flächen von $\Psi$ zwischen $M M$.

$$
\begin{array}{lr}
M: M=29017^{\prime} & \text { Berechnet } 29015^{\prime} \\
M: M=4945 & 4946
\end{array}
$$

$\begin{array}{llr}(3) \Phi=\{11.5 . \overline{1} \overline{6} .12\} \frac{1}{2} R \frac{8}{3} \quad & \Phi: \Phi=26^{\circ} 18^{\prime} & \text { Berechnet } 25^{\circ} 26^{\prime} \\ & \Phi: \Phi=6012 & 5932\end{array}$

Ghañarcillo. Mit $r$ Mbav. $\Phi$ mit grossen und sehr bestimmten Flächen an zwei Krystallen entwickelt (s. Fig. 12), welche jedoch unscharfe Bilder geben, bedingl durch eine Rundung der Flächen nach der Form $\{7.3 . \overline{0} \overline{0} . \overline{\}}$, mit welcher sie manchmal fast zusammenfallen.
(4) $n^{\prime}=\{8.3 . \bar{T} \bar{T} .2\} \frac{5}{2} R^{1}{ }_{5}^{1}$
$n^{\prime}: n^{\prime}=2902^{\prime}$
$r: n^{\prime}=37 \quad 6$
Berechnet $29^{\circ} 52^{\prime}$
3720

\begin{tabular}{|c|c|c|}
\hline$\{23.9 . \overline{3} \overline{2} .14\} R^{16}$ & $\{12.5 . \overline{1} \overline{7} .2\}-\frac{13}{2} R^{17} \frac{7}{3}$ & S. (19) \\
\hline$\{14.9 . \overline{2} \overline{3} .32\}_{3}{ }_{3}^{\frac{5}{2}} R^{23}$ S. (11) & $\{7.3 . \overline{1} \overline{0} .6\} \frac{2}{3} R \frac{5}{2}$ & S. (22) \\
\hline$\{8.14 . \overline{2} \overline{2} .9\}-\frac{2}{3} R \frac{11}{3}$ S. u. (14) & $\{21.16 . \overline{3} \overline{7} .17\}_{1}$ & S. u. \\
\hline$\}_{\frac{3}{4}} R_{\frac{4}{3}}$ S. น. $\oint 18 \mathrm{~A}(2)$ & $\{17.12 . \overline{2} \overline{9} .14\}_{1}^{5}$ & S. \\
\hline$R \frac{7}{5} \quad$ S. u. $\oint 18 \mathrm{~A}$ & $\{8.63 .71 .134\}$ & S. \\
\hline $\begin{array}{ll}5166\} \frac{2}{3} R \frac{3}{2} & \text { S. u. } \oint 18 \mathrm{~A} .\end{array}$ & $\{8.53 . \overline{6} \overline{1} .114\}-\overline{1}^{4}$ & \\
\hline. $\bar{T} .14\} \frac{4}{7} R \frac{7}{4}$ S. u. $\S$ & $\{8.43 .5 T .94\}-\frac{3}{9} \frac{5}{4} L$ & 9. \\
\hline & & 19. \\
\hline & & \\
\hline
\end{tabular}

Markirch. Glänzende aber gestreifte Flächen; mit $e a \alpha r$.

b. Zweifelh afte Formen, bisher nicht beobachlet.

Pyrargyrit.

$K=\{15.11 . \overline{2} \overline{6} .22\}_{11}^{2} R_{2}^{13} \quad b: K=53^{0} 2^{\prime} \quad$ Berechnet $53^{0} 5^{\prime}$

Andreasberg. Lineare Fläche in der Zone $B V$ mit den Flächen $x^{\prime} L B$. 
Andreasberg. Schmale Fläche zwischen $y Z: y Z=24^{0} 37^{\prime}$. Berechnet $24^{\circ} 30^{\prime}$.

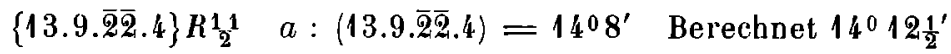

Andreasberg $;$ schmale Fläche zwischen $a v$.

\$ 12. Formen, welche dem Pyrargyrit und Proustit gemeinsam sind.

$$
\begin{aligned}
& \%=\{0001\} \quad \beta=\{21 \overline{3} 0\} \quad \gamma=\{53 \overline{8} 2\} \quad \tau=\{41 \overline{5} 0\} \\
& u=\{10 \pi 4\} \quad a=\{1+\overline{2} 0\} \quad \alpha=\{25 \overline{7} 3\} \quad \Delta=\{19.13 . \overline{3} \overline{2} .6\} \\
& r=\{10 \overline{1} 1\} \quad p=\{11 \overline{2} 3\} \quad d=\{12 \overline{3} 2\} \\
& e=\{01 \overline{1} 2\} \quad l=\{21 \overline{3} 4\} \quad y=\{32 \overline{5} 1\} \\
& s=\{02 \overline{2} 1\} \quad w=\{31 \overline{4} 5\} \quad P=\{15 \overline{6} 2\} \\
& b=\{10 \bar{T} 0\} \quad v=\{21 \overline{3} 1\} \quad \zeta=\{9.5 . \overline{1} \overline{4} .4\}
\end{aligned}
$$

Formen, welche nur am Proustit vorkommen:

$$
\begin{aligned}
& ? h=\{03 \overline{3} 2\} \quad M=\{35 \overline{8} 7\} \quad \Phi=\{11.5 . \overline{6} \dot{6} .12\} \\
& n^{\prime}=\{8.3 . \bar{\top} .2\} \quad \vartheta^{\prime}=\{59 \overline{7} 9\} \quad \Psi=\{43 \overline{7} 7\} .
\end{aligned}
$$

Der Rest der in $\S 4$ gegebenen Formen kommt allein am Pyrargyril vor.

\section{§ 13. Relative Häufigkeit der Formen.}

$$
\text { Pyrargyril. }
$$

An 127 Exemplaren, deren wichtigste Fundorte :

$\begin{array}{lr}\text { Harz } & 50 \\ \text { Sachsen } & 25 \\ \text { Mexico } & 19 \\ \text { Oesterreich etc. } & 9 \\ \text { Hiendelaencina } & 6 \\ \text { Laasphe } & 5\end{array}$

waren die gewöhnlichsten Formen :

$$
\begin{aligned}
& a=\{11 \overline{2} 0\} \text { an allen Stucken }=100 \% \\
& e=\{0172\}-87 \quad-\quad=64 \\
& q=\{16 \overline{7} 1\}-87 \quad-\quad=64 \\
& b=\{10 \pi 0\}-69-\quad=54 \\
& v=\{21 \overline{3} 1\}-58-=45 \\
& \iota=\{21 \overline{3} 4\}-54-\quad=42 \frac{1}{2} \\
& r=\{10 \pi 1\}-51 \quad-\quad=40 \\
& p=\{11 \overline{2} 3\}-22-=17 \\
& y=\{32 \overline{5} 1\}-.18-=14 \\
& u=\{10 \overline{1} 4\}-9 \quad=7
\end{aligned}
$$




\section{Proustit.}

An 60 Exemplaren, deren hauptsächlichste Fundorte

$\begin{array}{lr}\text { Chili } & 20 \\ \text { Sachsen } & 19 \\ \text { Oesterreich etc. } & 8 \\ \text { Mexico } & 4\end{array}$

sind, waren die gewöbnlichsten Formen :

$$
\begin{aligned}
& v=\{21 \overline{3} 1\} \text { an } 47 \text { Stucken }=78 \% \\
& a=\{11 \overline{2} 0\}-45-=75 \\
& e=\{01 \bar{T} 2\}-40-=67 \\
& s=\{02 \overline{2} 1\}-25-=42 \\
& r=\{10 \bar{T}-1\}-11-=18 \\
& b=\{10 \bar{T} 0\}-10-=17
\end{aligned}
$$

Andere häufig vorkommende Formen sind :

$$
\begin{aligned}
& \text { am Pyrargyrit } X Y \alpha \varphi w \gamma F E f, \\
& \text { am Proustit } \quad M \alpha .
\end{aligned}
$$

R e l h w isch berechnete die relative Häufigkeit der Formen der Rolhgiltigerze nach dem Vorgange I r b y's, indem er die Anzahl der Combinationen bei Lévy, in welchen die Formen wiederkehren, zählt. Nach dem, was im Vorhergehenden bezüglich der L é v y'schen Mittheilungen gesagt wurde,

\begin{tabular}{|c|c|c|}
\hline$a\{11 \overline{2} 0\} \infty P 2$ & $X\{11.1 . \overline{1} \overline{2} .1\} 10 R_{\overline{5}}^{6}$ & $Q\{14.4 . \overline{1} \overline{8} .13\})_{13}^{10} R^{9}$ \\
\hline$e\{01 T 2\}-\frac{1}{2} R$ & $V\{12.5 . \overline{1} 7.10\}_{\top \frac{7}{0}} A^{\frac{1}{7}}$ & $B\{4376\} \frac{1}{6} R 7$ \\
\hline$g\{16 \overline{7} 1\}-5 R_{5}^{7}$ & $L\{53 \overline{8} 6\} \frac{1}{3} R 4$ & $N^{\prime}\{17.15 . \overline{3} \overline{2} .2\} R 16$ \\
\hline$b\{10 \overline{4} 0\} \infty 0 R$ & $f\{05 \overline{5} 1\}-5 R$ & $\alpha\{25 \overline{7} 3\}-R \frac{7}{3}$ \\
\hline$v\{21 \overline{3} 1\} R 3$ & $d\{12 \overline{3} 2\}-\frac{1}{2} R 3$ & $E\{13 \overline{4} 1\}-2 R 2$ \\
\hline$t\{21 \overline{3} 4\}_{1}^{1} R 3$ & $F\{4.15 . \overline{1} 9.4\}-\frac{11}{4} R \frac{1}{1} \frac{9}{1}$ & $l\{45 \overline{9} 5\}-\frac{1}{5} R 9$ \\
\hline$r\{10 \bar{T} 1\} R$ & $\Gamma\{07 \overline{7} 2\}-72 R$ & $n\{41 \overline{5} 3\} R \frac{5}{3}$ \\
\hline$p\{11 \overline{2} 9\} \frac{2}{3} p 2$ & $U\{8.3 . \overline{1} 1.8\} \frac{5}{8} R \frac{1}{5}$ & $m^{\prime}\{6.5 . \overline{1} \overline{1} .7\} \frac{1}{7} R 11$ \\
\hline$y\{32 \overline{5} 1\} R 5$ & $D\{1.12 . \overline{1} 3.1\}-11 R_{1}^{13}$ & $P\{1, \overline{6} 2\}-2 R_{\frac{3}{2}}$ \\
\hline$u\{10 \bar{T} 4\} \frac{1}{4} R$ & $Y\{7.4 . \overline{1} 1.6\}\}_{1} R U_{3}$ & $T\{50 \overline{5} 2\} \frac{5}{2} R$. \\
\hline$s\{02 \overline{2} 1\}-\geqq R$ & $\gamma\{53 \overline{8} 2\} R 4$ & \\
\hline$w\{34 \overline{4} 5\} \frac{2}{5} R 2$ & $z\{43 \overline{7} 4\} \frac{1}{4} R 7$ & \\
\hline
\end{tabular}
ergiebt sich, dass eine solche Berechnung obne Werth ist.

\section{§ 14. Typische Formen (vergl. die sphär. Proj. auf Taf. IV).}

Es ist von Wichtigkeit, die typischen Formen, welche als glänzende, unabhängige Flächen auftreten, zu sondern von denjenigen, welche nur durch flächenreiche Zonen inducirt erscheinen, oder welche nur als schmale Flächen an Stelle von typischen Kanten auftreten.

$$
\text { Pyrargyrit. }
$$




\section{Proustit.}

$$
\begin{aligned}
& v\{21 \overline{3} 1\} R 3 \\
& a\{11 \overline{2} 0\} \infty P 2 \\
& e\{01 \bar{T} 2\}-\frac{1}{2} R \\
& s\{02 \overline{2} 1\}-2 R \\
& r\{10 \bar{T} 1\} R \\
& b\{10 \bar{T} 0\} \infty R
\end{aligned}
$$

$$
\begin{aligned}
& \alpha\{25 \overline{7} 3\}-R \frac{7}{3} \\
& M\{35 \overline{8} 7\}-\frac{2}{7} R 4 \\
& P\{15 \overline{6} 2\}-2 R \frac{3}{2} \\
& \vartheta^{\prime}\{52 \overline{7} 9\} \frac{1}{3} R 7 \\
& \Psi\{43 \overline{7} \overline{3}\} \frac{1}{4} R 7 \\
& \Phi\{11.5 . \overline{1} \overline{6} .12\} \frac{1}{2} R \frac{8}{3} .
\end{aligned}
$$

Vielleicht ist den typischen Formen des Pyrargyrits noch hinzuzufügen $\varepsilon \delta \eta$ (Klein) und $a^{\prime} W$ (Rethwisch, Seligmann).

\section{\$15. Rhomboëdrischer Charakter des Rothgiltigerzes.}

Der scharf ausgeprägle rhomboëdrische Gharakter (im Gegensatze zu hexagonaler Ausbildung) dieser Mineralien ist sehr auffallend. Sowohl am Pyrargyrit als am Proustit ist nicht ein einziger gut begrúndeter Fall des Vorkommens einer typischen Fläche irgend einer Form bekannt, zu welcher die Gegenform existirte (d. i. von $+m R n$ und $-m R n$ ).

Die einzigen angegebenen Fälle, mit ihren Autoren, sind in nachfolgender Tabelle zusammengestellt :

$\begin{array}{llll}r\{10 \overline{1}\} R & \text { Gewöbnlich } & Y^{\prime}\{01 \overline{1}\}-R & \text { Lé vy } \\ e\{01 \overline{1} 2\}-\frac{1}{2} R & \text { Gewöhnlich } & b^{\prime}\{10 \overline{1} 2\} \frac{1}{2} R & \text { Lévy } \\ d\{12 \overline{3} 2\}-\frac{1}{2} R 3 & \text { Sicher } & g\{21 \overline{3} 2\} \frac{1}{2} R 3 & \text { Lévy } \\ \eta\{5.10 . \overline{1} 5.8\}-\frac{5}{8} R 3 \text { Klein } & k\{10.5 . \overline{1} \overline{5} .8\} \frac{5}{8} R 3 & \text { Mohs, Groth } \\ q^{\prime}\{4.8 . \overline{1} 2.5\}-\frac{4}{5} R 3 & \text { Lévy } & \xi^{\prime}\{8.4 . \overline{2} .5\} \frac{4}{5} R & \text { Zippe } \\ C^{\prime}\{72 \overline{9} 2\} \frac{5}{2} R \frac{9}{5} & \text { Sella } & K^{\prime}\{27 \overline{9} 2\}-\frac{5}{2} R \frac{9}{5} & \text { Sella } \\ v^{\prime}\{42 \overline{6} 1\} 2 R 3 & \text { Sella } & x\{24 \overline{6} 1\}-2 R 3 & \text { Mohs } \\ E\{31 \overline{4} 1\}-2 R 2 & \text { Gewöhnlich } & \varrho\{13 \overline{4} 1\} 2 R 2 & \text { Zippe }\end{array}$

Alle rechts stehenden Formen sind aus fruher entwickelten Gründen zweifelhaft, mil Ausnahme von $g, k$ und $K^{\prime}$; wäbrend von den links stehenden Formen $q^{\prime}$ ebenso unsicher ist wie sein inverses $\xi^{\prime}$.

$k$ könnte, wenn es nur durch die Angaben von Mohs allein gestiatzt wäre, nicht als sichere Form betrachtet werden; dieselbe wird jedoch auch von Groth als am Pyrargyrit von Freiberg vorkommend angegeben (Mineraliensammlung der Universität Strassburg, S.64). Die Anwendung des Buchstabens $\eta$ an Stelle von $k$ in Groth's Beschreibung zeigt indessen, dass eine Verwechslung von $\frac{5}{8} R 3$ und $-\frac{5}{8} R 3$ eingetreten ist, wie denn auch Prof. B ü cking die Form an den Strassburger Krystallen nicht finden konnte*).

*) Prof. G roth hat mir spàter mitgetheilt, dass diese Form von ihm in der That nicht beobachtet wurde, und dass 1. c. S. 64 , Zeile 9 statt $\eta=+\frac{5}{8} R 3$ heissen soll $\eta=$ $-\frac{5}{8} R 3$ (welche Form, abgesehen vom Vorzeichen, der Form $\frac{1}{2} R_{\frac{1}{3}}^{11}$ sehr nahe stehl). 
$K^{\prime}$ muss, ungeachtet der Autorität Sell a's, als zweifelbafte Form angesehen werden, da sie in einer gekrümmten oder gestreiften Zone liegt. Es bleibt daher nur noch die Fläche $g$, von welcher Reflexe in der Zone $B V$ erhalten wurden, eine Thatsache, welche jedoch nicht genugt, um diese Form als sicher aufzustellen.

Die einzige Form von hexagonalem Aussehen, welche sich an diesen Mineralien findet, ist $p\{1 / \overline{2} 3\}{ }_{3}^{2} P 2$, welche als Deuteropyramide ihre eigene inverse ist; und gerade diese Form ist, obgleich sie zuweilen mit glänzenden, ebenen Flächen vorkommt, noch häufiger ersetzt durch zwei naheliegende Flächen (s. d. Taf. in $§ 18)$.

Der strenge Unterschied zwischen + und - Formen ist ausgezeichnet ersichtlich in den Hauptzonen. Die flächenreiche Zone zwischen $a r$ ist auf der negativen Seite einzig durch die Form $\alpha$ vertreten, welche zu keiner andern die inverse ist. Die Formenreihe zwischen $b E$ ist auf der positiven Seite einzig durch die beiden Formen $p^{\prime} X$ vertreten und die Reihe zwischen $V B$ auf der negativen Seite nur durch $t d P$.

Beim Kalkspath kommen von den durch Goldschmidt in seiner Zusammenstellung als sicher angenommenen Formen zweiundzwanzig in beiden Stellungen, positiv und negaliv, vor, nämlich sechs Rhomboëder, neun Deuteropyramiden $m P 2$ und sieben Skalenoëder $\pm R \frac{5}{3}, \pm R \frac{7}{3}, \pm R \frac{9}{5}, \pm R 2$, $\pm R 3, \pm R 5, \pm 2 R 3$. Merkwürdig ist, dass $\pm 2 R 3$ auch für Rothgiltigerz aufgefübrt wurde.

Es ist daher möglich, als ein allgemeines Gesetz für Pyrargyrit und Proustit auszusprechen, dass keine lypische Form in beiden Stellungen vorkommt. Ja, es darf sogar als wahrscheinlich bezeichnet werden, dass überhaupt keine Form in beiden Stellungen vorkommen kann.

\section{§16. Hemimorphismus.}

1) Proustit. - Der einzige Beweis für die Hemimorphie ist die bemiëdrische Entwicklung des Prismas $b\{10 T 0\}$ an den abwechsẹlnden Kanten von $a\{1 \mid \overline{2} 0\}$, und von $\tau\{41 \overline{5} 0\}$ an den anderen Kanten (von Streng) beschrieben (s. diese Zeitschr. 4, 323). Die Krystalle sind nie an beiden Seiten, ausgebildet und am aufgewachsenen Ende konnten entscheidende Formen bisher nicht gefunden werden.

2) Pyrargyrit. - An 124 Stücken des Materials im British Museum, an welchen die Formen sich mit Sicherheit erkennen oder messen liessen, konnten folgende directe Beweise erbracht werden:

52 Stücke baben Andeutungen von Flächen an beiden Krystallenden, davon haben

33 Stücke $q$ oder $q b$ am angewachsenen Ende und einige der Formen tevysf' uproTw $p$ Yld am freien Ende; 
8 haben $q$, sowie einige von den Formen $\alpha r n G v u s^{\prime} w^{\prime}$ am aufgewachsenen und einige der Formen tvIyepru am freien Ende.

8 zeigen folgende Combinationen:

Aufgewachsenes

Ende:

$r$

$r n$

$n \quad \cdots$

$n \Pi v$

$n \alpha$

$r n \Pi s^{\prime}$

$r \alpha n$

$\operatorname{ta} Y(?)$
Freies Ende:

vyqfpt I

$e$

$\operatorname{vtyrg}(?)$

$\beta q v t(?)$

$p$

$F y q X V \operatorname{Lr} \operatorname{te} \varphi B x^{\prime} \sigma g p \gamma Q p^{\prime}$

$v p$

$t v y$

3 zeigen folgende Combination:

a) $\operatorname{erq}(?)$ am aufgewachsenen, $q \alpha \vartheta S e Z^{\prime}$ am freien Ende.

b) $e r$ am einen, ert $\psi \rho \alpha \vartheta \varrho \pi q$ am andern Ende eines isolirten Krystalls.

c) $q$ am einen, $q r e v$ am andern Ende.

40 Slücke waren nur an einem einzigen Ende entwickelt und zeigten die Form $q$ in Gesellschaft von Flächen der Formen repw YECvyPsufXL

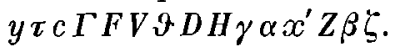

32 Stucke waren nur an einem Ende begrenzt, jedoch ohne Andeulung der Form $q$, sondern Combinationen der Formen evptvwfyry UXIVFsw $Y T d L \boldsymbol{x}^{\prime} \boldsymbol{m}^{\prime} \boldsymbol{A}$.

Es muss hinzugefügt werden, dass die Ausbildung beider Enden hierbei nicht an einem einzelnen Krystalle, sondern stets durch genaue Untersuchung der ganzen Stufe festgestellt wurde. Es muss daher aus diesen Beobachtungen gefolgert werden, dass entweder: (1) die angewachsenen Enden verschieden sind von den freien in Folge der verschiedenen Bedingungen, welche an beiden Enden herrschten, und $n \Pi \alpha s^{\prime}$ charakteristisch sind fur die ersteren, oder: (2) die beiden Enden wesentlich verschieden sind (wobei eines charakterisirt ist durch die Flächen $q$ ), aber die Krystalle nicht immer mit demselben Ende aufgewachsen sind.

Der erste Schluss wird gestitzt durch die Thatsache, dass $n$ und $\boldsymbol{I}$ niemals an dem frei ausgebildeten Ende gefunden wurden, und $s^{\prime}$ nur ein einzigmal, so dass diese Formen auf jeden Fall als für das aufgewachsene Ende des Krystalles charakteristische angesehen werden können. Andererseits führt die Art und Weise der Entwicklung von $q$ streng zu dem zweiten Schluss.

$q$ erscheint gewöbnlich nur als eine linienförmige oder sehr schmale Fläche, welche wenig mehr als eine Reihe von Streifen auf dem Prisma 
$a\{11 \overline{2} 0\}$ bildet. Diese Streifen sind nun stets (ausgenommen, wenn Gompli-

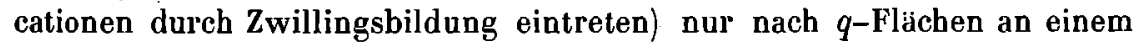
Ende des Krystalles gerichtet, wie dies für gewisse Fälle schon von Schuster gezeigt wurde, dessen diesbezaglichen Folgerungen identisch sind mit denjenigen, zu welchen ich aus ziemlich denselben Gründen gelangt war, ebe genannter Autor seine Arbeit veröffentlicht hatte.

Das Ende, zu welchem die Streifung nach $q$ binführt, ist gewöhnlich das aufgewachsene, bei den mexicanischen und einigen anderen Exemplaren ist es jedoch auch das freie Ende.

Diese Thatsachen beweisen, dass die beiden Enden des Pyrargyrits unterschieden werden mussen, und berechtigen zu folgender Eintheilung:

Ein Ende, welches im Allgemeinen charakterisirt ist durch die Form $q$, zeigt die Formen :
$n\{41 \overline{5} 3\}$
$\alpha\{25 \overline{7} 3\}$
$\Pi\{30 \overline{3} 2\}$
$s^{\prime}\{7.3 .1 \overline{0} .4\}$
$G\{1.9 . \overline{1} 0.1\}$
$w^{\prime}\{51 \overline{6} 4\}$

und mit aller Wahrscheinlichkeit auch :

$\begin{array}{lll}\vartheta\{2.15 . \bar{T} \overline{7} .8\} & \varrho\{27 \overline{9} 7\} & C\{11.4 . \overline{\bar{S}} \overline{5} .10\} \\ Y\{7.4 . \overline{1} .6\} & \varphi\{41 \overline{5} 6\} & u\{10 \overline{1} 4\} \\ S\{2.13 . \overline{1} \overline{5} .7\} & D\{1.12 . \bar{\top} 3.1\} & r\{10 \bar{T} 1\} \\ Z^{\prime}\{4.20 . \overline{2} \overline{4} .11\} & H\{15 \overline{6} 1\} & Z\{54 \overline{9} 1\} \\ \psi\{31 \overline{4} 2\} & P\{15 \overline{6} 2\} & f\{05 \overline{5} 1\} . \\ \pi\{8.23 . \overline{3} \bar{T} .18\} & & \end{array}$

Dieses Ende ist gewöbnlich das aufgewachsene, und sind für dasselbe charakteristisch die Flächen $n \Pi s^{\prime}$.

Es ist nicht ganz sicher, zu welchem Ende $m^{\prime}$ und die zwischen $V B$ eingeschlossene Flächengruppe zu rechnen ist*).

Das andere Ende zeigt die ubrigen am Pyrargyrit beobachteten Flächen. Die Prismen $\beta \tau$ gehören zu jenen Kanten von $a$, welche nicht abgestumpft werden, $f^{\prime}$ zu denen, welche durch $b$ abgestumpft und von $q$ begleilet sind.

Die Formen euptwrv und vielleicht auch $Y$ treten an beiden Enden auf, $q$ kann auch an jenem Ende auftreten, welches für gewöhnlich als das frei ausgebildete erscheint (s. Fig. 9). Diese Fläche erscheint aber niemals an beiden Enden gleichzeitig und die oben unter (a) und (c) angefuhrten Fälle sind vielleicht durch Zwillingsbildung veranlasst. Die Figuren 3 bis 6 sind Abbildungen von vier Andreasberger Krystallen, welche eine hemimorphe Ausbildung aufweisen, Fig. 1 dagegen stellt eine ideale Combination der wichtigsten, fur die beiden Enden charakteristischen Formen dar.

*) Nach Fig. 2 ist es wahrscheinlicb, dass die Zone $b_{1} V B e_{3}$ zu dem Ende gehört, welches allgemein als freies (unaufgewachsenes) angesehen wird.

Groth, Zeitschrift f. Krystallogr. XV. 
M. Schuster's Beobachtungen am Pyrargyrit von Andreasberg ergeben, dass eytvpv $r \omega$ charakteristisch fur das eine, und $q n P H \alpha w^{\prime}$ für das andere Ende sind; es ist ersichtlich, dass obiges Resultat in vollkommener Uebereinstimmung mit diesem ist. Seine Angabe, dass für das eine Ende spitze positive und stumpfe negative, fur das andere stumpfe positive und spilze negative Skalenoëder charakteristisch sind, erfordert eine Einschränkung, sie ist annähernd richtig, soweit als sie nur die spitzen Skalenoëder allein betrifft.

Es wurde versucht, miltelst des Thomson'schen Quadrantelektrometers irgend welche pyroëlekırische Eigenschaften des Pyrargyrits oder Proustits zu entdecken; es war jedoch bis jetzt unmöglich, eine Spannungsdifferenz an den beiden Krystallenden während der Dauer einer Temperaturänderung nachzuweisen.

Es ist jedoch zu beachten, dass die erwartete Eigenschaft vollständig verhulltt werden kann durch Zwillingsbildung, wenigstens beim Pyrargyrit.

Wir versuchten deshalb, Beweise für den Hemimorphismus zu erlangen, indem wir Krystalle ätzten und zwar mit: Salpetersäure, einer Mischung von Salpeter- und Weinsäure und mit Brom, ohne aber bis jetzt ein entscheidendes Resultat zu erlangen.

\section{\$ 17. Zwillingsverwachsungen.}

Im Verlaufe der Arbeit fand ich Beispiele unter beiden Mineralien für folgende funf $Z$ willingsgesetze :

\begin{tabular}{|c|c|c|c|}
\hline Pyrargyri & 1) 2 & & $u(1074$ \\
\hline & 2) & - & $r(10 \bar{\top} 1)$ \\
\hline & 3) & - & $a(1 \mid \overline{2} 0)$ \\
\hline & 4) & - & $o(0001)$ \\
\hline & 5) & - & $e(0 \mid \bar{T} 2)$ \\
\hline Proustit: & 1) & - & $u(10 \pi 4)$ \\
\hline & 2) & - & $r(10 \pi 1)$, \\
\hline & 4) & - & $o(0001)$ \\
\hline & 5) & - & $e(01 \bar{T} 2)$ \\
\hline
\end{tabular}

Beim Pyrargyrit ist das dritte Gesetz das allerhäufigste; nicht allein beinahe jedes Handstuck, sondern auch fast jeder Krystall zeigt Andeutungen desselben; das erste Gesetz ist ebenfalls sehr bäufig und an den meisten Stücken verwirklicht, das zweite und fünfte dagegen selten.

Beim Proustit ist andererseits das erste und zweile Gesetz gewöhnlich, dagegen die beiden andern selten.

$$
\text { 1) Zwillingsebene } u=\left\{10 T^{4}\right\} \frac{1}{4} R \text {. }
$$

Diese Zwillingsverwachsung ist in bewundernswerther Weise von $\mathrm{H}$ a idinger beschrieben worden und haben seine Abbildungen ibren Weg in alle Handbücher genommen. 
Die Krystalle sind derartig verwachsen, dass ein Paar Flächen von $e=\left\{01 T_{2}\right\}-\frac{1}{2} R$ in einem Individuum parallel sind, einem Flächenpạare derselben Form im anderen Individuum (Fig. 24). Gew̧öhnlich ragen aus einem grösseren Kryștalle eine Anzahl kleinerer heraus, indem alle drei Flächen der Form $u$ als Zwillingsebenen auftreten. Die freien Enden der Krystalle bilden gemeiniglich einen Winkel von $26^{\circ}$ mit einander (d. $h$. der Winkel zwischen den morphologischen Axen ist 25040' beim Pyrargyrit und $26^{\circ} 8^{\prime}$ beim Prouslit); in diesem Falle ist die Verwachsungsfläche, wie es im gewöhnlichen Sprachgebrauche heisst, senkrecht zur Zwillingsfläche (Fig. 24). Der weniger bäufige Fall, in welchem die freien Enden einen stumpfen Winkel von $154^{\circ}$ mit einander bilden, hat, wie man zu sagen pflegt, die Verwachsungsfläche parallel der Zwillingsfläche (Fig. 25). Beide Arten der Verwachsung kommen vor, doch ist die orstere häufiger als die letztere.

Da indessen die Verwachsungsfläche keine wirkliche Ebene ist, sondern die Krystalle sich unregelmässig durchdringen, so werden dieselben richtiger beschrieben als auf der gleichon Seite der Zwillingsebene ausgebildet im ersten Falle, im zweiten dagegen als auf entgegengesetzten Seiten jener Ebene. Da eine Verwachsung senkrecht zur Zwillingsebene schwer verständlich ist, so kann man auch annehmen, dass die Krystalle in Wirklichkeit nach einer Fläche von $f=\{0551\}-5 R$, welche beinahe senkrecht steht zu der Fläche $u$ oder zur Kante des Rhomboëders $e$, verzwillingt sind. Ist dies der Fall, dann müssen aber die benachbarten Flächen von $e$, welche nach der ersten Annahme in beiden Individuen parallel sein sollen, in Wirklichkeit einen einspringenden Winkel bilden und zwar von 51 $\frac{1^{\prime}}{2}$ beim Pyrargyrit und von $10411^{\prime}$ beim Proustit.

Zur Entscheidung dieser Frage wurde eine Anzahl Zwillingskrystalle gemessen mit dem Resultate, dass die anliegenden Flächen der Form $e$ nur sehr selten, wenn uberhaupt jemals, strenge parallel gefunden werden. Sie bilden gewöhnlich einen einspringenden, zuweilen auch einen ausspringenden Winkel, dessen Werth zwischen $\dot{g}^{0}$ und $9^{\circ}$ schwankt.

Diese Veränderlichkeit in der Position der Zwillingskrystalle zeigt in ausgezeichneter Weise eine Pyrargyritgruppe von Andreasberg, an welcher die relative Stellung von funf Krystallen bestimmt werden

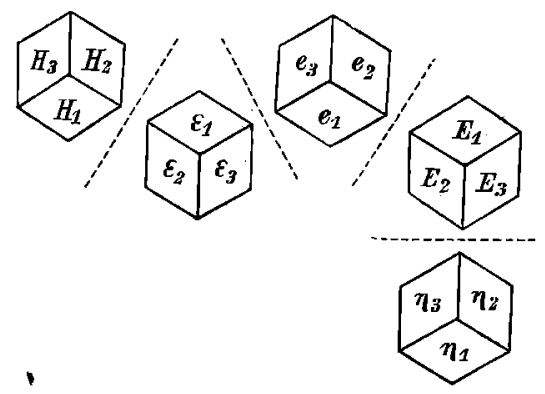
konnte und in nebenstebender Figur erläutert ist.

Diese Gruppe zeigt auch die Veränderlichkeit des Rhomboëderwinkels an einem und demselben Krystalle und die daraus fúr die genaụe Bestim- 
mung dieser Grösse sich ergebenden Schwierigkeiten. Die Flächen von $e\{01 T 2\}-\frac{1}{2} R$ sind in den fünf Krystallen bezeichnet mit resp. $e E \varepsilon \eta H$. Die Reflexbilder waren gut, aber nicht vollkommen.

Zwillingswinkel :

(a)

(c)

(d)

$$
\begin{array}{lr}
e_{1} E_{2}=2013^{\prime} \\
e_{2} E_{1}=1 & 54 \\
e_{1} \varepsilon_{3}=2 & 6 \\
e_{3} \varepsilon_{1}=4 & 13 \\
E_{3} \eta_{2}=0 & 55 \\
\varepsilon_{2} H_{1}=0 & 24
\end{array}
$$

Rhomboëderwinkel:

Mittel :

$$
\begin{aligned}
& e_{1} e_{2}=41058^{\prime} \\
& \left.\begin{array}{l}
e_{2} e_{3}=41 \quad 57 \\
e_{3} e_{1}=42 \quad 0
\end{array}\right\} e e=44^{058^{\prime}} \\
& E_{1} E_{2}=42 \quad 7 \\
& \left.E_{2} E_{3}=425\right\} E E=425 \\
& E_{3} E_{1}=42 \quad 2 \\
& \varepsilon_{1} \varepsilon_{2}=42 \quad 2 \\
& \left.\begin{array}{rlr}
\varepsilon_{2} \varepsilon_{3}=42 & 1 \\
\varepsilon_{3} \varepsilon_{1}=41 & 58
\end{array}\right\} \varepsilon \varepsilon=420 \\
& \eta_{3} \eta_{2}=42 \quad 9 \quad \eta \eta=42 \quad 9 \\
& H_{2} H_{1}=42 \quad 7 \quad H H=42 \quad 7
\end{aligned}
$$

Andere Beispiele, in welchen die Messungen genauer angestellt werden können als in dem obigen und sicher auf eine oder zwei Minuten zuverlässig sind, sind die folgenden:

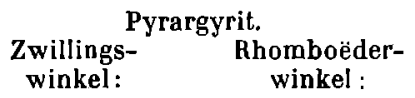

(1) $e_{1} E_{2}=2^{0} 10^{\prime} \quad e e=42^{\circ} 6^{\prime}$

$$
e_{2} E_{1}=224 \quad E E=425
$$

(2) $e_{1} E_{2}=1 \quad 3 \quad$ ee $=425$

$e_{2} E_{1}=1 \quad 4 \quad E E=425$

(3) $e_{1} E_{2}=133 \quad e e=426$

$e_{2} E_{1}=134 \quad E E=426$

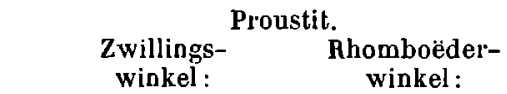

(1) $e_{1} E_{2}=0057^{\prime} \quad$ ee $=42046^{\prime}$

$e_{2} E_{1}=056 \quad E E=4246$

(2) $e_{1} E_{2}=229 \quad e e=4246$

$20 \quad E E=4246$

Es ist ersichtlich, dass diese Werthe nicht erkennen lassen, ob $u$ oder $f$ Zwillingsebene ist; sie fuhren jedoch zu der weiteren Frage, ob nicht eine gewisse Regelmässigkeit in der Abweichung der Krystalle von der wahren Zwillingsstellung zn finden sei. Auf ähnliche Abweichungen ist bei anderen Mineralien aufmerksam gemacht worden*), jedoch wurden dieselben nicht genauer beschrieben.

Beim Pyrargyrit und Proustit ist nun der Winkel $e E$ auf einer Seite der den beiden Krystallen gemeinsamen Kante gemeiniglich gleich dem auf der anderen Seite. Dies kann nun auf zweierlei Art stattfinden : a) entweder ist die Zone $e_{1} E_{2}$ nahezu parallel der Zone $e e$ und $E E$, oder mit anderen Worten: die Kanten $e e$ und $E E$ sind nahezu parallel, und ein Krystall kann betrachtet werden als von der wirklichen Zwillingsposition

*) Des Cl o i zea ux, Manuel de Minér. 2, 109. 
abweichend durch eine Drehung um die, beiden Individuen gemeinsame Rhomboëderkante; - oder b) die Zone $e_{1} E_{2}$ ist nabezu senkrecht zur Rhomboëderkante $e e$ und $E E$, mit anderen Worten: die Flächen $a$ sind nahezu parallel, und ein Krystall kann angeseben werden als abweichend von der wirklichen Zwillingsstellung durch Drehung in der Prismenfläche $a$, welche beiden Individuen gemeinsam ist.

Fasst man die wirkliche Zwillingsstellung und die Abweichungen von derselben in einen einzigen Ausdruck zusammen, so kann die Zwillingsverwachsung folgendermassen beschrieben werden : Zwei Krystalle sind derart mil einander verwachsen, dass sie eine Fläche $a$ und eine in dieser Fläche gelegene Kante $e e$ gemeinschaftlich haben, und die Abweichungen von dieser Lage hervorgerufen werden entweder durch eine geringe Neigung der beiden Flächen $a$ unter.Erhaltung des Parallelismus der gemeinsamen Kante, oder einer geringe Neigung der in Rede stehenden Kanten, während die Flächen $a$ parallel bleiben*).

Auf gleiche Weise kann die Zwillingsverwachsung am Bournonit (Zwillingsebene: (110)) ausgedruckt werden durch die Angabe, dass beide Krystalle eine Fläche (110) und die Kante (Zonenaxe) [001], d. i. die verticale, in dieser Fläche gelegene Axe gemeinsam haben. Die Abweichungen von der wirklichen Zwillingsposition (beschrieben Min. Mag. 1884, 6, 77**) unter dem Titel "Unregelmässige Verwachsung (1) können entweder einer Neigung nur der Fläche (110) oder nur der Kante [001] zugeschrieben werden. Beim Rothgiltigerz zeigt es sich in den Fällen, in denen die Abweichung weder durch eine einfache Drehung um die Kante $e e$, noch durch eine solche um die Normale zur Fläche $a$ ausgedruckt werden kann, öfter, dass der Winkel $e_{1} E_{2}$ zwischen einem Paar anliegender Flächen genau doppelt so gross ist, als zwischen dem anderen Flächenpaare $e_{2} E_{1}$ (s. Beispiel (6) oben).

Anderweitige Beobachtungen ưber die Veränderlichkeit der Orientirung von $Z$ willingskrystallen scheinen nicht gemacht worden zu sein, so dass es nicht möglich ist zu sagen, welche der hier beschriebenen Erscheinungen sich auch bei anderen Mineralien finden.

Zwillingslamellirung. Obgleich die directen Messungen an Zwillingskrystallen die Frage nicht entscheiden konnten, ob $u$ oder $f$ als Zwillingsebene zu betrachten sei, so wird doch durch die Thatsache, dass zuweilen die Verwachsung mit der Fläche $u$ selbst stattfindet, jedes Bedenken, welches gegen die erstere Deutung erhoben werden konnte, beseitigt $\left.{ }^{* * *}\right)$.

*) M. Schuster, l. c.

**) Referirt diese Zeitschr. 11, 175.

***) Entscheidende Beweise werden weiter unten gegeben. 
Die ausgeprägteste Form dieser Verwachsung besteht in einer Zwillingslamellirung nach der Fläche $u$, welche eine ganz gewöhnliche Erscheinung am Pyrargyrit ist, bisher aber noch nicht beschrieben wurde. Manche Pyrargyritkrystalle zeigen bei sorgfältiger Untersuchung vereinzelte Linien von äusserster Feinheit, welche gewöhnlich nahe am Ende des Krystalles quer uber die Prismen- und Skalenoëderflächen verlaufen. Diese Linien können zuweilen rings um den Krystall verfolgt werden als ein System von dreierlei Streifen parallel den drei Rhomboëderflachen $u$. Zuweilen geben dieselben am Goniometer schwache Reflexe, welche entweder 1) einer ungefähr $22^{0}$ zu den Prismenflächen geneigten Ebene, oder 2) einer Fläche parallel $u$ entsprechen; der Winkel $22^{\circ}$ ist der zwischen den Prismenflächen zweier nach $u$ verzwillingter Krystalle.

Es kann daher kein Zweifel sein, dass diese Linien feine Zwillingslamellen parallel den Flächen $u$ darstellen und dass dieselben begrenzt sind an ihren Kanten durch das Prisma $a$ und an ihrer Oberseite durch die Fläche $u$, welche sonst eine ausserordentlich seltene Form ist*). Die Lamellen können endlich durch den ganzen Krystall hindurch verfolgt werden als feine, die Bruchflächen durchsetzende Linien; die Krystalle zeigen jedoch keinerlei Neigung sich nach den Lamellen abzusondern. Zuweilen haben dieselben mehr das Ansehen von Gleitflächen.

Secundäre $Z$ willingsbildung. In einigen Fällen spielt $u$ unzweifelhaft die Rolle einer Gleitfäche. Ein Pyrargyritkrystall von Andreasberg zeigt dies besonders deutlich. Derselbe ist augenscheinlich entweder während oder nach der Krystallisation gepresst worden und ist nun durchzogen von einer Reihe deutlich sichtbarer $Z$ willingslamellen, an welchen die Flächen des Prismas hinreichend breit sind, um einen bestimmten Reflex zu geben. Eine gute Messung desselben ergab den Winkel zwischen den Flächen zweiẹr abwechselnder Lamellen zu $22^{\circ} 8^{\prime}$, der berechnete beträgt $22{ }^{\circ} 11^{\prime}$.

Versuche, die Zwillingsbildung kanstlich am Pyrargyrit hervorzurufen, waren nicht von Erfolg getrönt (um einen gleichförmigen und homogenen Druck zu erzeugen, wurde $u$. a. der Krystall in ein Glasrohr mit Wasser eingeschlossen und dieses in eine Kältemischung gebracht). Am Proustit wurden Zwillingslamellen parallel $u$ nicht beobachtet.

Zusammenhang $z$ wischen Zwillingsbildung und Hemimorphie. Was die Stellung der beiden Zwillingskrystalle beim Proustit anbelangt, so kann dieselbe gleich gut bezeichnet werden durch jede der folgenden zwei Annahmen:

1) Zwillingsaxe normal zur Fläche $u$,

*) Diese Lamellen mögen auch die Angabe veranlasst haben, dass die Prismenflächen parallel der Fläche $g$ gestreift seien (Re thw iscb, l. c. S. 80). 
2) Zwillingsaxe parallel der Kante $e$.

Ziehen wir aber noch den hemimorphen Charakter des Pyrargyrit in Betracht, so haben wir ein Kriterium, welches uns gestattet, zwischen diesen beiden Erklärungsarten, mindestens für genanntes Mineral, sicher zu entscheiden.

Fig. 14, Taf. V stellt einen Pyrargyritkrystall $(a e)$ in normaler Stellung dar; $q$ bezeichnet jenes Ende des Krystalles, gegen welches die Streifung auf den Prismenflächen $a$ convergirt, um das Skalenoëder $q$ zu bilden (wie in Fig. 5 dargestellt).

Eine halbe Drehung um die Normale zu $u$ als Zwillingsaxe bringt den Krystall in die Stellung von Fig. 20, während eine halbe Drehung um die Kante $q r$ ihn in die Stellung der Fig. 21 bringt.

Fig. 22 zeigt die vollständige Durchwachsung der beiden Krystalle im erslen Falle und Fig. 23 im zweiten Falle.

Ist nun 1) der richtige geometrische Ausdruck fur das Zwillingsgeserz, so muss $q$ an jenen Krystallenden erscheinen, welche einen spitzen Winkel miteinander bilden und der Zwilling ist symmetrisch nach einer Fläche, welche senkrecht zur Kante $q r$ steht; ist dagegen 2) die richtige geometrische Erklärung, so muss $q$ an jenen Enden erscheinen, welche unter stumpfem Winkel gegen einander geneigt sind und der Zwilling ist symmetrisch nach einer Fläche parallel $u$.

Nun hat eine sorgfältige Untersucbung dieser Zwillinge ergeben, dass, mit Ausnabme jener Fälle, wo durch Auftreten des dritlen Zwillingsgeselzes Complicationen zu Stande kommen, die Verwachsung stets die der Fig. 22 entsprechende ist, und dass also die Erklärung "Zwillingsaxe normal zur Fläche $u\{10 T 4\}$ « der wirklich riehtige geometrische Ausdruck für diese Zwillingsverwachsung ist.

Was die Art der Verwachsung und Ausbildung bei diesem Geselze anbelangt, so sind die Krystalle entweder zu beiden Seiten der Zwillingsfläche, wie in Fig. 25, oder nur auf einer Seite derselben, wie in Fig. 24, ausgebildet; der letztere Fall ist jedoch bei Weitem der häufigere.

Die frei entwickelten Enden der Zwillingskrystalle, wie es in Fig. 24 dargestellt ist, können entweder jene sein, welche die Andeutungen der Flächen $q$ besitzen oder diejenigen, an denen sie nicht auftreten.

$$
\text { 2) Zwillingsebene } r=\{10 \pi 1\} R \text {. }
$$

Dieses Gesetz ist weil häufiger beim Proustit, als beim Pyrargyrit. Dieselben Betrachtungen, wie beim ersten Gesetze, können auch hier angestellt werden, mit dem einzigen Unterschiede, dass die beiden Individuen nun nahezu einen recbten Winkel mit einander bilden.

Eine Verwachsung analog der in Fig. 22 abgebildeten würde resultiren 
bei einer halben Drehung um die Normale zu $r$, und eine der Fig. 23 analoge durch eine halbe Drehung um die in der Fläche $r$ gelegene Kante $s s$.

Eine Untersuchung der wenigen nach diesem Gesetze verzwillingten Pyrargyritkrystalle lässt keinen Zweifel aufkommen, dass nur die erstere Verwachsungsart allein den wirklich vorkommenden Zwillingen entspricht und dass dieselben geomelrisch beschrieben werden können durch genannten Ausdruck: Zwillingsaxe die Normale zu $r$, oder Zwillingsebene die Fläche $r$.

Beim Proustit ist kein Unterschied zu machen zwischen diesem Falle und einer Hemitropie nach der in der Fläche $r$ gelegenen Kante $s s$.

Nach der von Streng gegebenen Beschreibung und Abbildung von Proustitzwillingen von Chã̃arcillo sind deren Hauptaxen unter 940 $18^{\prime}$ geneigt, oder mit anderen Worten : die beiden Krystalle auf entgegengesetzten Seiten der Zwillingsebene gelegen. Bei allen Stucken, welche ich zu untersuchen Gelegenheit batte, lagen dagegen die beiden Individuen auf derselben Seite der Zwillingsebene, so dass ihre Hauptaxen einen Winkel von $8^{\circ} 42^{\prime}$ bilden. Beide Fälle sind leicht zu unterscheiden; im ersteren wưrden die schärferen Polkanten $(s)$ des vorherrschenden Skalenoëders $v$ einander gegenüberliegen, während im letzteren Falle stets die stumpfen Kanten einander zugekehrt sind, wie es in Fig. 27 dargestellt ist.

Beim Proustit finden sich die beiden ersten Zwillingsgesetze öflers combinirt, indem der Raum zwischen zwei nach $r$ verzwillingten Individuen durch einen dritten Krystall, welcher zu einem der beiden ersten nach $u$ verzwillingt ist, ausgefullt wird (s. Fig. 27).

Lamellare Zwillingsbildung nach diesem Gesetze ist beim Pyrargyrit sehr selten (wenn uberhaupt existirend); sie ist jedoch vorhanden, wenngleich selten, beim Proustit von verschiedenen Localitäten (s. S treng).

3) Zwillingsebene $a=\{11 \overline{2} 0\} \infty P 2$.

Die nach diesem Gesetze verzwillingten Krystalle wurden früber beschrieben als Zwillinge nach der Basis, und die Abbildungen, in welchen der Zwilling angedeutet ist, durch die unterbrochene Entwickelung des Prismas $b\{10 T 0\} \infty R$ an den abwechselnden Kanten des Prismas $a\{11 \overline{2} 0\}$, sind in allen Handbuchern zu finden. Die richtige Erklärung und Bezeichnung gewisser Andreasberger zu diesem Typus gehöriger Krystalle ist erst neuerdings von M. Sc huster gefunden worden, und seine Schlüsse werden völlig bestätigt durch die vorliegende Bearbeitung des Materials des British Museum, welche unabhängig davon zum selben Resultat geführt hat.

Die Figuren 14 bis 19, schon gezeichnet vor dem Erscheinen der Schuster'schen Arbeit, mögen dazu dienen, die Natur dieser Verwachsungen zu erklären. Max Schuster scheint dieses Gesetz als selten und 
nur ausnahmsweise vorkommend zu betrachlen und stellt die Existenz von Zwillingen nach der Basis oder nach dem Prisma $\{10$ T0\} $\infty R$ nicht in Abrede. Nach meinen Beobachtungen ist das in Rede stehende Gesetz jedoch ausserordentlich häufig, obgleich oft nicht in die Augen fallend, und fast alle durch Zwillingsbildung nach der Basis oder nach $b$ erklärten Fälle sind in Wirklichkeit als Zwillinge nach dem Prisma $a$ zu erklären.

Gehen wir vom einfachen Krystalle in normaler Stellung (Fig. 14) aus und unterscheiden dasjenige Ende, gegen welches die nach der Fläche $q$ gerichtete schiefe Streifung auf dem Prisma verläuft mit dem Buchstaben $q$, so bringt :

1) Eine halbe Umdrehung um die Verticalaxe, die Normale zur Basis (0001), den Krystall in die Position von Fig. 15. Eine Verwachsung zweier Krystalle in dieser Stellung nach der Basis wurde nun allerdings das trigonale Prisma $b$, welches an denselben Kanten des Prismas $a$ mit der Fläche $q$ vorkommt, alternirend am oberen und unteren Ende des Krystalles zur Ausbildung bringen; aber die, durch die schiefe Streifung indicirte Form $q$ würde nur an einem einzigen Ende der Krystalle orscheinen.

2) Eine halbe Umdrehung um die Kante zwischen $a$ und der Basis (normal zum Prisma b) bringt den Krystall in die Position von Fig. 16. Eine Verwachsung nach der Basis wurde einen Zwilling ergeben, in welchem die $q$-Flächen symmetrisch an dem oberen und unteren Ende vertheilt wären, aber das mit demselben vergesellschaftete Prisma $b$ wurde nur an abwechselnden Kanten des zusammengesetzen Krystalles als trigonales Prisma erscheinen.

3) Eine halbe Umdrehung um die Kante zwischen dem Prisma $b$ und der Basis (normal zu dem Prisma a) bringt den Krystall in die Position von Fig. 17. Eine Verwacbsung nach der Basis ergiebt einen Zwilling wie in Fig. 18, wenn die $q$-Enden der Krystalle nach aussen gerichtet, und Fig. 19, wenn sie nach innen gerichtet sind. Diese Zwillingsverwachsung ist.von Schuster als neu beschrieben; sie ist indessen, wie schon erwähnt, bereits fruher von $\mathrm{Naumann}$ abgebildet und beschrieben worden als »zwillingsartige Verwachsung(. (S. oben $§ 1$ 1.)

Zufolge meinen eigenen Beobachtungen ist der letzlgenannte der drei Fälle fast der einzige in der Natur verwirklichte, so dass also beinahe alle Zwillinge des Pyrargyrit mit parallelen Axen zu beziehen sind auf das Gesetz

$$
\text { Zwillingsebene } a=\{11 \overline{2} 0\} \infty P \text {. }
$$

Die Zwillingskrystalle können ibre $q$-Enden entweder nach aussen oder nach innen gerichtet haben.

Es ist eine Eigenthumlichkeit dieser Zwillinge, dass die so verwachsenen Krystalle das Aussehen von einfachen, holoëdrischen Individuen besitzen, weon dieselben nicht Flächen längs der Zwillingsnaht aufzuweisen 
liaben, welche dem nach innen gerichteten Ende angehören; sonst muss der Zwilling gleichartige Endigung haben und der hemimorphe Gbarakter ist vollständig versteckt.

Beim Pyrargyrit sind die Kennzeichen zur Unterscheidung der beiden verschiedenen Enden gegeben in der Streifung nach $q$, in der Entwicklung des Prismas $b$, oder in den weiter oben erwähnten Flächen, welche die Form $q$ zu bègleiten pflegen.

Es ist deswegen möglich, dass dieselbe Art der Zwillingsbildung auch an anderen rhomboëdrischen Mineralien vorkommt, aber aus Mangel an Kennzeichen der erwähnten Art bislang nicht aufgefunden wurde und dadurch auch ihr hemimorpher Gharakter verhilltt wird. Es wurden die Turmaline des British Museum nach dieser Richtung hin untersucht, jedoch ohne Erfolg; indess hat $\mathrm{K}$ und $\mathrm{t}^{*}$ ) mittelst der Methode der Bestäubung gezeigt, dass in Schnitten von Turmalin senkrecht zur Axe Theile existiren, welche die analogen Pole nach oben und andere, welche sie nach unten gekehrt haben, so dass auch bei diesem Mineral die Existenz einer Zwillingsbildung ähnlich der oben beim Pyrargyrit beschriebenen nicht unmöglich scheint.

Bei den Pyrargyritzwillingen findet die Verwachsung nie parallel der Basis statt, wie in Fig. 18 und 19, sondern entweder 1) unregelmässig, 2) parallel $u, 3$ ) parallel $r$ oder 4) parallel dem Prisma $a$. Die einzelnen Krystalle können einander ferner in jeder denkbaren Weise durchdringen.

Manchmal ist die Durchdringung eine so regelmässige, dass jede Fläche des Prismas $a$ durch eine verticale Zwillingsnaht in zwei Hälften getheilt ist, wie in Fig. 26. In diesem Falle kann der Krystall nur durch eine auusserst sorgfällige Untersuchung von einem wirklich einfachen boloëdrischen Krystalle unterschieden werden.

Zuweilen bildet ein Krystall eine, den andern theilweise umgebende Hulle.

Die Streifungen, wie sie in Fig. 14 bis 26 angedeutet sind, werden hervorgebracht von dünnen Platten, parallel dem Prismà $a$, welche auf die Prismenflächen aufgelagert; dieselben sind an ihren Kanten nach einem Ende bin begrenzt von den Flächen $q n$ oder $q v$, und weil sie sich öfters in umgekehrter oder Zwillingsstellung zu dem centralen Krystall befinden, so ist es oft schwer zu bestimmen, welches Ende des letzteren in Wirklichkeit dasjenige ist, welches durch die Form $q$ charakterisirt ist.

Eine solche Zwilllingstafel, wie sie an einem Krystall von Mexico beobachtet wurde, ist in Fig. 13 abgebildet. Eine nur fluchtige Untersuchung eines solchen Krystalles könnte zu dem Schluss führen, dass das obere Ende des Krystalles dasjenige ist, zu welchem $q$, das hier mit den Flächen $r p$ ver-

*) S. diese Zeitschr. 8, 534. 
gesellschaftet wäre, gehörl. In Wirklicbkeit dagegen gehört $r p$ zum einen Ende des Krystalles und $\alpha v q$ zum andern, nur an der Zwillingstafel entwickelten Ende. Dies ist in der Figur ebenfalls angedeutet durch die Streifung, welche gegen das untere Ende zu $q$-Flächen zusammenläuft.

Die Streifung parallel der Fläche $q$ wird oft durch diese lamellare Zwillingsbildung hervorgerufen, welche, wenn man nicht die allergrösste Sorgfalt auf die Deutung der Streifung verwendet, leicbt zu einer vollständig falschen Orientirung des Krystalles führen kann. Die Streifung auf den Prismenflächen $a$ läuft, wenn sie von Zwillingsbildung herruhrt, parallel den Flächen $a v q \alpha$. Dieselbe Ursache bringl eine sehr feine Zwillingslamellirung parallel den Prismenkanten hervor, welche zuweilen als eine verticale Reihe von feinen Linien, einigermassen ähnlich den beim ersten Gesetz beschriebenen, parallel $u$, beobachtet werden kann.

Das dritte Gesetz kann, wie ersichtlich, in Gemeinschaft mit jedem der beiden ersten auftreten; man beobachtet es jedoch nie in Verbindung mit dem zweiten Gesetze. Krystalle, welche nach dem ersten Gesetze, d. h. nach einer Fläche von $u$, verzwillingt sind, zeigen dagegen häufig, in Folge von Zwillingsbildung nach dem dritien Gesetze, sehr verwickelte Verbältnisse (wie oben erwähnt), indem jeder Krystall zusammengesetzt und scheinbar holoëdrisch ist und dadurch die Orientirung desselben unmöglich wird. Auf die gleiche Schwierigkeit stösst man bei Entscheidung der Frage, ob Quarzzwillinge mil geneigten Axen zu beschreiben sind als solche nach einer Fläche (z. B. von $\{1 \mid \sqrt{2} 2\})$ oder nach der in dieser Fläche gelegenen Kante, da jedes Individuum ein zusammengesetzter Krystall ist. Beim Pyrargyrit ist dagegen, sobald die Verwachsung nicht verwickelt wird durch das Hinzutreten des dritten Gesetzes, der Zwilling stets erklärbar durch Hemitropie un die Fläche $u$.

$$
\text { 4) Zwillinge nach } o=\{0001\} 0 R \text {. }
$$

Obgleich dieses Gesetz in allen Handbuichern als ein für den Pyrargyrit gewöhnliches angegeben wird, habe ich dennoch während meiner Untersuchung keinen Fall von anscheinender Zwillingsbildung nach der Basis gefunden, der sich nicht, bei sorgfältiger Untersuchung, als zum dritten Gesetz gehörig erwiesen hätte. Erst ganz neuerdings beobachtete ich solcbe aus Andreasberg. Ob hier aber wirklich $\{0001\} 0 R$ als $Z$ willingsebene zu betrachten sei oder $\{10 \bar{T} 0\} \infty R$, kann nicht entschieden werden, weil jedes der beiden Individuen selbst wieder ein $Z$ willing mit parallelen Axen (nach $a$ ) ist.

Am Proustit ist dieses Zwillingsgesetz an einem einzigen Beispiel im British Museum aufgefunden worden, und zwar an einem Krystall einer Gruppe von matten, aber wohlausgebildeten, skalenoëdrischen Krystallen von Marienberg. Derselbe ist in Fig. 28 abgebildet.

Es muss bemerkt werden, dass ohne directe Beweise fur die Hemi- 
morphie des Proustits es unmöglich ist, zu sagen, ob dieses Gesetz zu beschreiben ist als Zwilling nach $o\{0001\}$ oder nach $b\{10 T 0\}$. Siehe Fig. 15 und 16.

$$
\text { 5) Zwillings ebene } e=\{01 \overline{1} 2\}-\frac{1}{2} R \text {. }
$$

Dieses Gesetz fand sich an zwei Proustitstufen von Markirch i. E. und zwar an prismatischen Krystallen, in welche Zwillingslamellen paralle] $e$ eingelagert sind; ferner an einer Gruppe skalenoëdrischer Proustitkrystalle von Freiberg.

Auch ein Pyrargyrit-Exemplar von Freiberg in der L udlam'schen Sammlung scheint nach diesem Gesetz verzwillingt zu sein, die beiden Individuen stehen, wie gewöhnlich, auf der gleichen Seite der Zwillingsfläche.

$$
\text { 6) Zwillingsebene } b=\{10 T 0\} \infty R \text {. }
$$

Dieses Gesetz, welches auf der Autorität $\mathrm{H}$ a i ding e r's beruht (Ed. J. Sc. 1824, 326, Fig. 14-17), bedarf einer erneuten Untersuchung, um zu bestimmen, ob dasselbe Gesetz so auszudrucken ist, oder ob die betreffende Verwachsung beschrieben werden muss als Zwilling nach 0 mit Zusammensetzung parallel $b$.

\section{\$18. Hauptzonen (gekritmmte und gestreifte) *).}

Die gestreiften Zonen aller Mineralien verdienen besondere Aufmerksamkeit. Wenn die am Goniometer erbaltenen Reflexe nicht auf das Sorgfältigste kritisirt und unterschieden werden, so werden nur zu leicht (als das Mittel mehrerer Bilder) gerade solche Flächen als typische angenommen, welche als solche gar nicht vorkommen, sondern ersetzt sind durch vicinale Flächen. Es ist dies vielfach von frühern Beobachtern gethan worden; so ist z. B. die Fläche $A=\{12.7 . \overline{1} \overline{9} .14\}$, von S ell a aufgeführt, fast unabänderlich ersetzt durch die Flächen $L=\{53 \overline{8} 6\}$ und $x^{\prime}=\{7.4 .1 T .8\}$ in der Gruppe zwischen $V B$ und ist keineswegs die typische Fläche in dieser Region des Krystalles. Wird andererseits allen reflectirenden Flächentheilen gleiches Gewicht beigelegt, so wird die Zone von einer continuirlichen Reihe von Flächen ausgefullt, welche schwer zu interpretiren sind.

Einige Autoren**) weisen darauf hin, dass es zur Bestimmung der Indices von vicinalen Flächen und Flächen in gestreiften Zonen nicht genugt, das Mittel aus verschiedenen Messungen zu nehmen, sondern dass nur die einzelnen Messungen an jedem Krystalle entscheiden können, welche von diesen Flächen, die als locale Entwickelungen zu betrachten sind, vor-

*) Eine flächenreiche Zone ist gestreift, wenn die Flächen einspringende Winkel zu bilden streben; sie ist gekrümmt, wenn dieselben ausspringende Winkel bilden.

**) Hintze, diese Zeitschr. 11, 234 . 
Beiträge zur kenntniss des Pyrargyrit und Proustit. Bibliothe ks

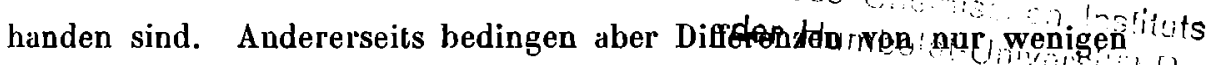

Minuten in den Messungen eine grosse Differenz im Symbol einer Flächè Berlin

mit hohen Indices. Da nun sogar die Flächen der Rhomboëder $\{10 \bar{T}\}\}$ und $\{01 \overline{1} 2\}$, wie oben gezeigt wurde, selbst wenn dieselben vollkommen glatt und glänzend und nicht durch vicinale Flächen verwickelt sind, Störungen ihrer Orientirung erlitten haben, so können Flächen mit sehr hohen Indices mit keinem Symbole belegt werden, wenn deren Messungen nicht nachweislich auf drei bis vier Minuten richtig sind.

Es schien deshalb bei der Behandlung der gestreiften Zonen am Pyrargyrit besser, zu bestimmen, welche Flächen mit einfachen Symbolen in diesen Zonen nicht entwickelt, sondern ersetzt sind durch Gruppen von benachbart gelegenen Flächen, welche Symbole diese letzteren anzunehmen pflegen und schliesslicb diese zu vergleichen mit den typischen Formen, durch welche die Zone oder ein Theil derselben gelegentlich vertreten ist.

$\mathrm{Zu}$ diesem $\mathrm{Zwecke}$ wurde die ganze Reihe der am Goniometer erhaltenen bestimmten Reflexe einer gestreiften Zone an einer Anzahl von Krystallen reducirt auf Winkelabstände von irgend einer Fläche in dieser Zone*); dann wurden dieselben sämmtlich in Columnen geordnet, so dass die gleichen Werthe auf horizontale Linien fallen. Es zeigt sich alsdann, dass die Ablesungen in woblbestimmte Gruppen zerfallen; das Mittel jeder Gruppe wurde zur Zusammenstellung einer neuen Reihe genommen, welche eine erste Annäherung zu den Flächen der Zone (Vicinalflächen der ersten Ordnung ?) darstellt; die individuellen Schwankungen innerbalb jeder Gruppe (Vicinalflächen der zweiten Ordnung) können nicht in Termen der Indices ausgedruckt werden und müssen deshalb für jetzt vernachlässigt werden.

Ein Vergleich der so gefundenen Winkel mit denen der typischen Formen, welche als glänzende Flächen frei von Streifungen auftreten, zeigt, dass die neue Columne alle typischen Flächen enthält und ausserdem noch andere, welche, wenn sie mit Regelmässigkeit wiederkehren und innerbalb nur kleiner Grenzen schwanken, als wahre Flächen der Zone betrachtet werden müssen und, wie zu hoffen ist, einiges Licht auf die Gesetze zu werfen im Stande sind, welche die Entwicklung dieser complicirten Zonen beherrschen.

Die flächenreichsten Zonen beim Pyrargyrit sind:

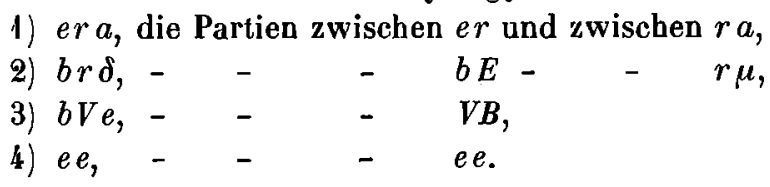

*) Es wurde Sorge getragen, dass die Messungen unter verschiedener Incidenz wiederholt wurden, so dass die wirklichen Flächen entsprechenden Reflexe ausgewăhlt werden konnten. 
Oscillatorische Combinationen oder gestreifte Flächen w'urden ferner beobachtet beim Pyrargyrit zwischen $b X, e \alpha, \alpha s \alpha$ und in der Nachbarschaft von $Y$ in der Zone $r Y$; beim Proustit zwischen $e s$ und an den Prismenkanten. Sella hat in seiner Abhandlung (Nuovo Cimento $1856,4,103$ ), welche offenbar das Ergebniss seiner Studien am Rothgiltigerz ist, es als ein Gesetz aufgestellt, dass, wenn eine Zone durch eine Reihe gestreifter Flächen charakterisirt, $d$. h. eine wichtige Zone am Krystalle ist, die conjugirte Fläche (d. i. die Fläche, welche dasselbe Symbol wie die Zone hat) sich durch Vollkommenheit und Glanz auszeichnet. Diese Angabe wird jedoch bei eingehenderer Untersuchung des Minerals nicht bestätigt, indem die vier, den ohen erwähnten vier Zonen entsprechenden Flächen folgende Bescbaffenheit besitzen :

1) $r=\{1011\}$ ist vollkommen und glänzend,

2) $p=\{11 \overline{2} 3\}$ ist gewöhnlich gestreift, zuweilen glänzend,

3) $=\{22 \overline{4} 3\}$ existirt nicht,

4) $s=\{02 \overline{2} 1\}$ ist glänzend und vollkommen,

während gestreifte Zonen entsprechend evta, den vier vollkommensten Flächen beider Mineralien, nicht aufzufinden sind.

Die nachstehenden Tabellen geben in den ersten drei Columnen die Messungsresultate an den oben erwähnten Zonen in solchen Fällen, wo dieselben als Reihen gestreifter Flächen entwickelt sind. Die letzte Columne giebt die berechneten Winkel für jene Flächen, welche aufgestellt wurden auf Grund unabhängiger Messungen an wohlausgebildeten Flächen.

A. Beobachtungen*).

1) Zone er a. Gestreift, er zuweilen gekrummt. Zahl der gemessenen Zonen $\mathbf{4 3 .}$

\begin{tabular}{|c|c|c|c|c|c|c|}
\hline$e:(h, k 0)$ & Grenzen : & & Zahl: & $\begin{array}{l}\text { Bekannte } \\
\text { Form: }\end{array}$ & $\{h k 0\}$ & Berechnet \\
\hline $4^{0} 59^{\prime}$ & ... & & 1 & $\Lambda$ & $\{540\}$ & $4^{0} 34^{\prime}$ \\
\hline 717 & $\ldots$. & & 1 & $z^{\prime}$ & $\{430\}$ & 5 52 \\
\hline 1032 & $\ldots$. & & 1 & $\nu$ & $\{320\}$ & 811 \\
\hline 121 & $11054^{\prime}-12^{0}$ & $7^{\prime}$ & 3 & $\omega$ & $\{530\}$ & 1011 \\
\hline 1254 & $1242-13$ & 2 & 9 & $\Sigma$ & $\{13.7 .0\}$ & 1210 \\
\hline 1325 & $1311-134$ & 44 & 24 & $p$ & $\{210\}$ & 1328 \\
\hline 1343 & $1334-134$ & 47 & 7 & .. & .. & .. \\
\hline 1411 & $14 \quad 1-141$ & 17 & 3 & .. & .. & .. \\
\hline
\end{tabular}

*) In den folgenden Tabellen sind statt der Bravais'schen Indices die Millerschen aufgenommen, weil aus diesen die betreffenden Zonenverhältnisse ohne Weiteres ersichtlich sind und sie den weiterhin folgenden Berechnungen zu Grunde gelegt werden müssen. 


\begin{tabular}{|c|c|c|c|c|c|}
\hline$e:(h k 0)$ & Grenzen: & Zahl: & $\begin{array}{l}\text { Bekannte } \\
\text { Form: }\end{array}$ & $\{h k 0\}$ & Berechnet \\
\hline $14^{\circ} 33^{\prime}$ & $14^{0} 30^{\prime}-14^{0} 37^{\prime}$ & 3 & $\ldots$ & . & .. \\
\hline 1450 & $1437-1459$ & 6 & $\Pi^{\prime}$ & $\{13.6 .0\}$ & $14050^{\circ}$ \\
\hline 1516 & $15 \quad 12-1520$ & 2 & .. & .. & .. \\
\hline 1551 & $1544-15.57$ & 3 & $\Gamma^{\prime}$ & $\{730\}$ & 162 \\
\hline $16 \quad 34$ & $1630-16^{\circ} 41$ & 4 & .. & .. & .. \\
\hline 170 & $\ldots$ & 1 & $r$ & $\{520\}$ & 177 \\
\hline 1730 & $1723-1731$ & 4 & $\boldsymbol{\tau}^{\prime}$ & $\{830\}$ & 185 \\
\hline 1821 & $180-1851$ & 7 & $\lambda$ & $\{11.4 .0\}$ & 1832 \\
\hline 1940 & $1926-205$ & 16 & $t$ & $\{310\}$ & 1946 \\
\hline 22 25 & $\ldots$ & 1 & $e^{\prime}$ & $\{10.3 .0\}$ & 219 \\
\hline 2240 & $\ldots$ & 1 & $\vartheta^{\prime}$ & $\{720\}$ & 2146 \\
\hline 2310 & $\ldots$. & 2 & $w$ & $\{410\}$ & $23 \quad 19$ \\
\hline 2336 & $\cdots$ & 1 & .. & . & .. \\
\hline $24 \quad 19$ & $\ldots$ & 1 & .. & .. & .. \\
\hline $25 \quad 24$ & $259-2532$ & 丂 & $\varphi$ & $\{510\}$ & 2536 \\
\hline 311 & $\ldots$ & 1 & $\xi$ & $\{610\}$ & 2710 \\
\hline 3330 & $33 \quad 19-33 \quad 48$ & כ & $G^{\prime}$ & $\{810\}$ & 2912 \\
\hline $34 \quad 20$ & $3412-3427$ & 2 & $\therefore$ & $\therefore$ & .. \\
\hline 3538 & $3527-3541$ & 14 & $\cdot r$ & $\{100\}$ & $35 \quad 41$ \\
\hline $36 \quad 4$ & $36 \quad 0-36 \quad 8$ & 2 & $\begin{array}{l}w^{\prime} \\
n\end{array}$ & $\begin{array}{l}\{50 T\} \\
\{40 T\}\end{array}$ & $\begin{array}{ll}47 & 8 \\
50 & 8\end{array}$ \\
\hline 4326 & $\ldots$ & 1 & $\psi$ & $\{30 \pi\}$ & 5510 \\
\hline 617 & $\ldots$. & 1 & $s^{\prime}$ & $\{70 \overline{3}\}$ & 6054 \\
\hline $\begin{array}{ll}65 & 12\end{array}$ & $6449-6543$ & 9 & $v$ & $\{20 T\}$ & 657 \\
\hline $65 \quad 48$ & $\cdots$ & 1 & .. & .. & .. \\
\hline 6620 & $6611-6628$ & 2 & .. & .. & .. \\
\hline 6722 & $6719-6724$ & 2 & $\therefore$ & $\therefore$ & .. \\
\hline $68 \quad 27$ & $\ldots$ & 1 & $\zeta$ & $\{90 \overline{5}\}$ & 6819 \\
\hline 6940 & $6929-6950$ & 2 & .. & . & .. \\
\hline $70 \quad 44$ & $\cdots$ & 1 & $\gamma$ & $\{50 \overline{3}\}$ & $70 \quad 49$ \\
\hline $71 \quad 12$ & $71 \quad 11-7114$ & 3 & .. & .. & .. \\
\hline $72 \quad 37$ & $7230-7244$ & 2 & .. & .. & $\therefore$ \\
\hline $73 \quad 35$ & $7334-7336$ & 2 & $I^{\prime}$ & $\{17.0 .1 T\}$ & 7324 \\
\hline 7357 & $\ldots$ & 1 & .. & .. & .. \\
\hline $74 \quad 28$ & $74 \quad 17-74 \quad 47$ & 11 & $y$ & $\{30 \overline{2}\}$ & $74 \quad 27$ \\
\hline $75 \quad 5$ & $\begin{array}{llll}75 & 2 & -75 & 18\end{array}$ & 8 & .. & .. & .. \\
\hline 7532 & $7530-7534$ & 2 & $\Delta$ & $\{19.0 . \overline{1} \overline{3}\}$ & 7522 \\
\hline $75 \quad 58$ & $7556-7558$ & 3 & .. & .. & .. \\
\hline $76 \quad 21$ & $7616-7628$ & 4 & $\Omega$ & $\{10.0 . \overline{7}\}$ & $76 \quad 12$ \\
\hline 7738 & $7731-7744$ & 2 & $x$ & $\{70 \overline{5}\}$ & 7656 \\
\hline
\end{tabular}




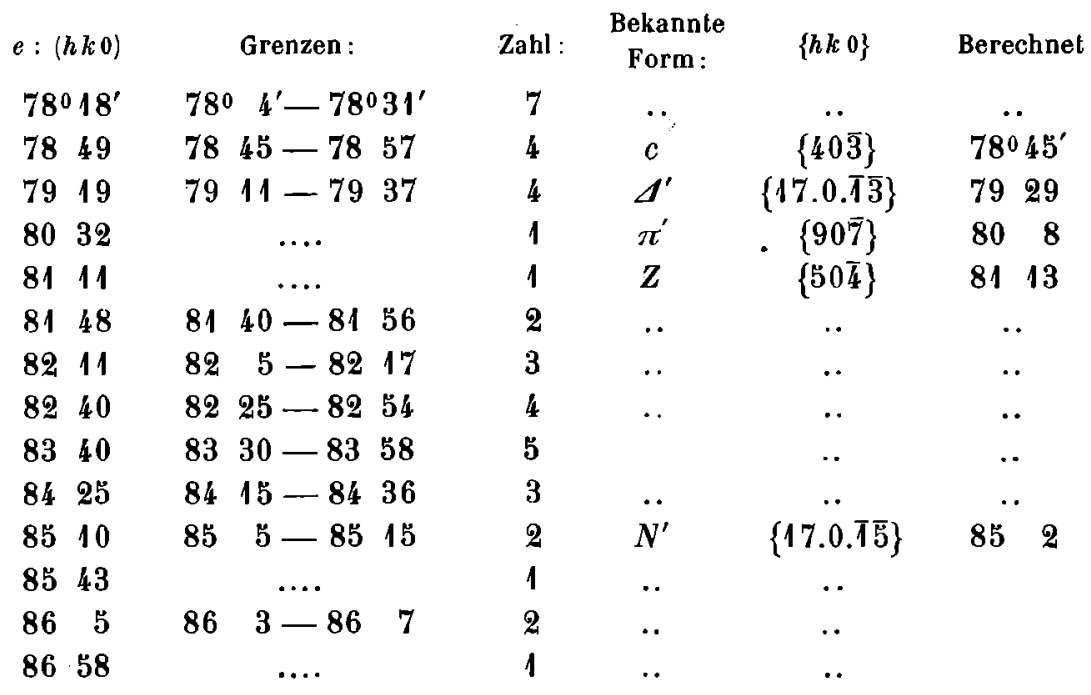

2) Zone $b r \delta$. Gewöhnlich gekrümmt.

a. Serie von $b$ nach $E$. Zahl der gemessenen Zonen 12.

\begin{tabular}{|c|c|c|c|c|c|c|}
\hline$b:(h k l)$ & & Grenzen: & Zahl : & & $\{h k l\}$ & Berechnet: \\
\hline $6^{0} 19^{\prime}$ & $6^{0}$ & $3^{\prime}-6^{0} 46^{\prime}$ & 3 & $D$ & $\{54 \overline{8}\}$ & $6^{0} 23^{\prime}$ \\
\hline 730 & & $\ldots$. & 1 & .. & .. & .. \\
\hline 743 & & ... & 1 & $G$ & $\{43 \overline{6}\}$ & 822 \\
\hline 1037 & 10 & $13-1046$ & 3 & .. & .. & .. \\
\hline 1145 & 11 & $22-1158$ & 3 & .. & .. & .. \\
\hline 1217 & 12 & $3-1296$ & 5 & $q$ & $\{32 \overline{4}\}$ & 128 \\
\hline 1241 & & $\ldots$ & 1 & .. & .. & .. \\
\hline 1318 & & .... & 1 & .. & .. & .. \\
\hline 1438 & 14 & $28--14 \quad 45$ & 4 & $H$ & $\{8.5 . \overline{1} \overline{0}\}$ & 1416 \\
\hline 152 & & $\ldots$ & 2 & .. & .. & .. \\
\hline $15 \quad 12$ & 15 & $11-1013$ & 2 & .. & .. & .. \\
\hline $15 \quad 24$ & 15 & $22-1525$ & 2 & $N$ & $\{53 \overline{6}\}$ & 1537 \\
\hline 1550 & 15 & $39-162$ & 3 & .. & $\cdot \cdot$ & . \\
\hline 1640 & & $\ldots$ & 2 & .. & .. & . \\
\hline 1653 & & $\cdots$ & 1 & .. & .. & .. \\
\hline 1720 & 17 & $19-1721$ & $\boldsymbol{Z}$ & $\Omega^{\prime}$ & $\{74 \overline{8}\}$ & 1715 \\
\hline 1731 & 17 & $28-1733$ & 3 & .. & .. & ..' \\
\hline 1747 & 17 & $43-1755$ & 7 & .. & .. & .. \\
\hline 189 & 18 & $3-1813$ & 6 & $F$ & $\{9.5 . \overline{1} \overline{0}\}$ & 1812 \\
\hline 1823 & 18 & $21-1826$ & 3 & $\boldsymbol{K}^{\prime}$ & $\{13.7 .1 \overline{4}\}$ & 1916 \\
\hline 1843 & 18 & $36-1853$ & 4 & $\boldsymbol{E}$ & $\{2 \mid \overline{2}\}$ & 2146 \\
\hline
\end{tabular}


b. Serien zwischen $r \mu$ (s. unten).

$\begin{array}{ccccc}r:(h k l) & \text { Grenzen: } & \text { Zahl : } & \{h k l\} & \text { Berechnet: } \\ 4041^{\prime} & 4024^{\prime}-4^{0} 50^{\prime} & 3 & \{23.2 . T\} & 4040^{\prime} \\ 526 & 521-531 & 2 & \{20.2 . \overline{1}\} & 521 \\ 622 & 610-637 & 3 & \{17.2 . \overline{1}\} & 616 \\ 811 & 743-849 & 3 & \{13.2 . \overline{1}\} & 88\end{array}$

Diese Gruppe gestreifter Flächen kam an der Ecke des Rhomboëders $r\{10 \bar{T} 1\}$ an einem Pyrargyritexemplare von Freiberg vor, welches die Combination des Prismas $a$ mit $q$ und $b$ und die Endflächen $r t$ zeigt.

3) Zone bVe. Im Allgemeinen gekrummt. Zabl der gemessenen Zonen 16.

\begin{tabular}{|c|c|c|c|c|c|c|}
\hline$b:(h k l)$ & & Grenzen : & Zahl: & Bekannte Form & & Berechnet: \\
\hline $39014^{\prime}$ & 390 & $2^{\prime}-39^{\circ} 27^{\prime}$ & 9 & $V$ & $\{13.1 . \overline{4}\}$ & $39^{0} 8^{\prime}$ \\
\hline 3941 & & & 1 & & & .. \\
\hline 4034 & & & 1 & & .. & .. \\
\hline $41 \quad 13$ & & $\ldots$ & 1 & .. & .. & .. \\
\hline 4326 & 43 & $23-43 \quad 32$ & 3 & $g$ & $\{7 \mid \overline{2}\}$ & 4320 \\
\hline $44 \quad 28$ & 44 & $14-4442$ & 2 & .. & .. & .. \\
\hline 4522 & 45 & $13-4536$ & 5 & $F^{\prime}$ & $\{40.7 . \bar{T}\}$ & $45 \quad 19$ \\
\hline $45 \quad 42$ & 45 & $30-4554$ & 2 & .. & .. & .. \\
\hline 4623 & 46 & $2-46 \quad 47$ & 12 & $x^{\prime}$ & $\{25.5 . \overline{7}\}$ & 4621 \\
\hline 4654 & & $\ldots$ & 1 & $A$ & $\{15.3 . \overline{4}\}$ & $46 \quad 49$ \\
\hline 4728 & 47 & $7-4746$ & 11 & $L$ & $\{19.4 . \overline{5}\}$ & 4726 \\
\hline $\begin{array}{ll}48 & 10\end{array}$ & 48 & $0-48 \quad 16$ & 5 & $\Xi$ & $\{27.6 . \overline{7}\}$ & $\begin{array}{ll}48 & 7\end{array}$ \\
\hline $48 \quad 48$ & & $\ldots$ & 1 & .. & .. & .. \\
\hline 4937 & 49 & $24-4954$ & $\breve{b}$ & $\sigma$ & $\{41 T\}$ & 4942 \\
\hline 5024 & 50 & $1-50 \quad 43$ & 4 & .. & .. & .. \\
\hline 5058 & 50 & $54-5137$ & 3 & .. & . & .. \\
\hline $5 \mathscr{2} \quad 5$ & 51 & $51-5225$ & 11 & $B$ & $\{17.5 . \overline{4}\}$ & 529 \\
\hline 532 & & $\ldots$ & 1 & $K$ & $\{21.6 . \overline{5}\}$ & 535 \\
\hline
\end{tabular}

4) Zone $e$. Sichere Messungen waren in dieser Zone nicht zu erlangen.

B. Indices der Flächen ingestreiften Zonen.

Die obigen Tabellen entbalten eine Reihe sorgfältig ausgewählter Messungen und mögen in späterer Zeit einmal als Daten zu einer allgemeinen Discussion gestreifter Zonen von Nutzen sein.

M. Sch u ster war in seiner mühsamen Arbeit über die Vicinalflächen des Danburits bestrebt zu zeigen, wie ihre Regelmässigkeit in Termen der Indices ausgedruckt werden kann, während andererseits $\mathrm{H}$ in $\mathrm{lz}$ e die Ansicht vertritt, dass die Vicinalflächen nur als locale Veränderung anzusehen 
sind und mit gleichẹr Richtigkeil in Termen ihrer directen Winkelbeziehungen ausgedrückl werden können; so sind beispielsweise eine Reihe von Vicinalflächen am Cölestin zu einander unter gleichen Winkeln geneigt. Im vorliegenden Falle mag es genügen, einige Regelmässigkeiten hervorzuheben, welche einige Theile der flächenreichsten Zonen des Pyrargyrits charakterisiren.

Diese Regelmässigkeit kann entweder in Termen der Indices oder der Winkel ausgedruckt werden, jedoch nicht in einer Reihe directer Winkeldifferenzen, wie beim Cölestin; sie zeigt desbalb ein in der inneren Structur des Krystalles begrundetes Gesetz der Progression an und nicht bloss ein oberflächliches und locales Phänomen. Die zur Andeutung dieser Regelmässigkeit unten gewählten Functionen sind nur mit Rucksicht auf den bequemen Gebrauch zur Rechnung aufgestellt und können leicht in die von Schuster oder Websky gebrauchten Bezeichnungen umgewandelt werden.

1) Zone era. Hier durchläuft die Function $\frac{h+k}{h-k}=\frac{\operatorname{tang} \theta}{\operatorname{tang} e r}$ (oder das anharmonische Verhältniss der vier Flächen $e, r,(h k 0), a)$, wenn $\theta$ den Winkel $e:(h k 0)$ bedeutet, eine Reihe von Brüchen, in welchen verschiedene Zablen in verschiedenen Theilen der Zone eine wichtige Rolle spielen. Für die dicht gedrängte Region zwischen $p t$ ist der Werth von $h-k 7$ oder ein Vielfaches davon, zwischen $y c$ ist er $\mathbf{5}$ oder dessen Vielfaches. Dies wird aus folgender Tabelle ersichtlich:

$\begin{array}{lcl}e:(h k 0) & (h k 0) & \frac{\operatorname{tang} \theta}{\text { lang } e r} \\ 12053^{\prime} & (29.15 .0) & \frac{7}{2^{2}} \\ 1328 & (210) & \frac{7}{21}=\frac{1}{3} \\ 1347 & (55.27 .0) & \frac{1}{4} 4 \\ 147 & (27.13 .0) & \frac{7}{20} \\ 1428 & (53.25 .0) & \frac{14}{39} \\ 1450 & (13.6 .0) & \frac{7}{19} \\ 1513 & (51.23 .0) & \frac{7}{37} \\ 162 & (730) & \frac{14}{35}=\frac{2}{5} \\ 1629 & (12.5 .0) & \frac{7}{17} \\ 1657 & (47.19 .0) & \frac{14}{33} \\ 1727 & (23.9 .0) & \frac{7}{16} \\ 1832 & (11.4 .0) & \frac{7}{15} \\ 1946 & (310) & \frac{7}{14}=\frac{1}{2}\end{array}$

Die obige Reihe kann geprüft werden durch eine Anzahl sehr guter Messungen, welche an zwei ungewöhnlich vollkommenen, zwei verschiedenen Stufen entnommenen Krystallen erhalten wurden. 


\begin{tabular}{|c|c|c|}
\hline$e:(h k 0)$ & Grenze : & Zabl : \\
\hline $12057^{\prime}$ & $12053^{\prime}-12058^{\prime}$ & 4 \\
\hline 1327 & $1321-1332$ & 5 \\
\hline $13 \quad 45$ & $1344-1349$ & 3 \\
\hline $14 \quad 49$ & $\cdots$ & 1 \\
\hline 1633 & $\ldots$ & 1 \\
\hline 1941 & $1939-1943$ & 6 \\
\hline
\end{tabular}

Der dicht gedrängle Theil der Zone zwischen $y c$ ist zu vergleichen mit nachstehenden berechneten Werthen:

$\begin{array}{lcc}e:(h 0 k) & (h 0 k) & \frac{\text { tang } \theta}{\text { tang } e r} \\ 74027^{\prime} & (30 \overline{2}) & 25=5 \\ 75 \quad 1 & (31.0 . \overline{2} T) & \frac{25}{5}=5 \\ 7533 & (16.0 . \bar{T} T) & \frac{27}{5} \\ 763 & (33.0 . \overline{2} \overline{3}) & 28 \\ 7630 & (17.0 . \bar{T} \overline{2}) & 5 \\ 7744 & (37.0 . \overline{2} \overline{7}) & \frac{29}{5} \\ 7826 & (39.0 . \overline{2} \overline{9}) & 5 \\ 7845 & (40 \overline{3}) & \frac{34}{5} \\ 7921 & (21.0 . \overline{1} \overline{6}) & 35=7 \\ & & \frac{37}{5}\end{array}$

2) Zone $b E$. Hier können wir die Function $\frac{h+5 k}{h-k}$, welche gleich ist dem Verhältniss $\frac{\operatorname{tang} b r}{\operatorname{tang} \theta}$ (oder dem anharmonischen Verhältniss der vier Flächen $b,(h k l), r,(5 T 2))$, worin $\theta=b:(h k l)$ ist, zu Grunde legen und finden dann die nachstehenden Winkel, berechnet aus einer um Differenzen von Siebenteln fortschreitenden Reihe, sehr genau mit den gemessenen Winkeln ubereinstimmend.

\begin{tabular}{|c|c|c|}
\hline$b:(h k l)$ & $(h k l)$ & $\frac{\operatorname{tang} b r}{\operatorname{tang} \theta}=\frac{h+5 k}{h-k}$ \\
\hline $6^{0} 23^{\prime}$ & $(54 \overline{8})$ & $\frac{175}{7}=25$ \\
\hline 735 & $(13.10 . \overline{2} \overline{0})$ & $147=21$ \\
\hline 741 & $(30.23 . \overline{4} \overline{6})$ & $\frac{145}{7}$ \\
\hline $10 \quad 34$ & $(10.7 . \overline{1} \overline{4})$ & $\frac{105}{7}=15$ \\
\hline 1146 & $(4.3 .29 . \overline{5} \overline{8})$ & $\frac{94}{7}$ \\
\hline 128 & $(32 \overline{4})$ & $\frac{91}{7}=13$ \\
\hline 1241 & $(61.40 . \overline{8} \overline{0})$ & $\frac{87}{7}$ \\
\hline 137 & $(17.11 . \overline{2} \overline{2})$ & $84=12$ \\
\hline 1438 & $(55.34 . \overline{6} \overline{8})$ & $7_{7}^{75}$ \\
\hline 150 & $(18.11 . \overline{2} \overline{2})$ & $\frac{73}{7}$ \\
\hline $15 \quad 12$ & $(107.65 . \overline{1} \overline{0} \overline{0})$ & 72 \\
\hline
\end{tabular}




$\begin{array}{llc}b:(h k l) & (h k l) & \frac{\operatorname{tang} b r}{\operatorname{tang} \theta}=\frac{h+5 k}{h-k} \\ 15025^{\prime} & (53.32 . \overline{6} \overline{4}) & \frac{71}{7} \\ 1550 & (52.31 . \overline{6} \overline{2}) & \frac{69}{7} \\ 1645 & (50.29 . \overline{5} \overline{8}) & \frac{65}{7} \\ 17 \quad 0 & (33.19 . \overline{3} \overline{8}) & \frac{64}{7} \\ 1715 & (74 \overline{8}) & \frac{63}{7}=9 \\ 1731 & (97.55 . \overline{1} \overline{1} \overline{0}) & \frac{62}{7} \\ 1747 & (48.27 . \overline{5} \overline{4}) & \frac{61}{7} \\ 184 & (95.53 . \overline{1} \overline{0} \overline{6}) & \frac{60}{7} \\ 1821 & (47.26 . \overline{5} \overline{2}) & \frac{59}{7} \\ 1839 & (31.17 . \overline{3} \overline{4}) & \frac{58}{7}\end{array}$

Es ist hier demnach, wenigstens zwischen der Position von (18.11. $\overline{2} \overline{2})$ und $(31.17 . \overline{3} \overline{4})$, der Werth von $h-k$ ein Vielfaches von 7 und die Function $\frac{h+5 k}{h-k}$ schreitet um gleiche Incremente fort.

3) Zone $b V e$. Die zwischen $V B$ gelegene Flächengruppe fand ich an gewissen Exemplaren von Andreasberg (wahrscheinlich aus der Grube Morgenröthe stammend), welche denselben Habitus besitzen, wie die von Phillips abgebildeten Krystalle.

Diese Zone ist die verwickelste an dem Mineral; doch kann kein Zweifel existiren, dass

$$
V=\{13.1 . \overline{4}\}, x^{\prime}=\{26.5 . \overline{7}\}, L=\{19.4 . \overline{5}\}, B=\{17.5 . \overline{4}\}
$$

sicher bestimmte typische Flächen dieser Zone bilden; die ubrigen sind vielleicht durch diese inducirt.

Die Gruppe ist immer nur durch spärliche Flächen ausgedehnt uber $B$ hinaus auf der einen und $V$ auf der andern Seite (die früher gegebene zweifelbafte Fläche \{21.6.5) wurde nur einmal beobachtet).

Es ist möglich, dass die Zonen, welche diese Reihe von Flächen mit der Fläche $v\{20 \pi\}$ verbinden, auch die Flächengruppe zwischen $\varphi\{510\}$ und $\omega\{530\}$ entbält; wenn dieses der Fall ist, so kann die Zone $V B$ beschrieben werden als die Projection der Zone $r e$ von der Fläche $v$ aus.

Dieses ist in der nachfolgenden Tabelle angedeutet, in welcher die erste Columne die Symbole von Flächen zwischen $r$ und $e$ enthält, während die zweite ihre Projectionen von $v$ aus auf die Zone $b e$, und die zweite und dritte Columne die correspondirenden Symbole und Winkel in der Zone be enthält. 


\begin{tabular}{|c|c|c|c|}
\hline \multirow{3}{*}{$\begin{array}{c}\text { Zone re. } \\
\text { Symbol: } \\
(510)\end{array}$} & \multicolumn{2}{|c|}{ Zone $b e$. } & \\
\hline & Symbol: & Winkel: & \\
\hline & $(13.1 . \overline{4})$ & $39^{\circ} \quad 8^{\prime}$ & $V$ \\
\hline$(920)$ & (23.2.7) & $\begin{array}{ll}39 & 47\end{array}$ & \\
\hline$(410)$ & $(10.1 . \overline{3})$ & 4037 & \\
\hline$(19.5 .0)$ & $(47.5 . \overline{1} \overline{4})$ & 412 & \\
\hline$(310)$ & $(7 / \overline{2})$ & 4320 & $g$ \\
\hline$(11.4 .0)$ & $(25.4 .7)$ & 44. 24 & \\
\hline$(23.9 .0)$ & $(51.9 . \overline{4})$ & 4524 & \\
\hline$(4.7 .19 .0)$ & $(103.19 . \overline{2} \overline{8})$ & 4553 & \\
\hline$(12.5 .0)$ & $(26.5 . \overline{7})$ & 4621 & $x^{\prime}$ \\
\hline$(730)$ & $(15.3 . \bar{k})$ & $46 \quad 49$ & $A$ \\
\hline$(940)$ & $(19.4 . \overline{5})$ & 4726 & $L$ \\
\hline$(13.6 .0)$ & $(27.6 . \overline{7})$ & 487 & $\Xi$ \\
\hline$(27.13 .0)$ & $(55.13 . \overline{1} \overline{4})$ & 4856 & \\
\hline$(210)$ & $(4 \cdot \pi)$ & $49 \quad 42$ & $\sigma$ \\
\hline$(29.15 .0)$ & $(47.15 . \overline{4})$ & $50 \quad 27$ & \\
\hline$(15.8 .0)$ & $(29.8 . \overline{7})$ & 519 & \\
\hline$(950)$ & $(17.5 . \overline{4})$ & 529 & $\boldsymbol{B}$ \\
\hline
\end{tabular}

Es scheint hier möglich, obgleich die wichtigen Flächen $L B$ nicht ihre entsprechenden in der Zone er haben, dass das allgemeine Gesetz der Progression in dieser Reihe abgeleitet werden kann aus dem der Zone er. In diesem Falle werden, da die Projection einer Fläche $\{h k 0\}$ das Symbol $\{3 h-2 k, k, k-h\}$ hat, die vicinalen Flächen der Zone $V B$ in ihrem letzten Index ein Vielfaches von 7 haben.

\section{c. Position vongestreiften und gekrummten Flächengruppen.}

1) Die Position der Gruppe $V-B$ ist bezeichnet durch eine eigenthumliche Erscheinung.

Wenn $\nabla$ der Punkt ist, bei welchem die Zone $b V e$ sich am nächsten der Fläche $r$ nähert, sodass $b \nabla$ senkrecht zu $V B$ ist, so ist $b \nabla=45^{\circ} 35^{\prime}$; nun ist dies aber genau das Centrum der Gruppe $V-B$, denn es sind die Bögen $V \nabla=6^{038^{\prime}}, B \nabla=6^{0} 31^{\prime}$. Daher ist der dichteste Theil der Zone $b e$ genau die Region, wo sie sich am meisten der Fläche des Rhomboëders $r$ nähert, und sie dehnt sich gleichweit zu beiden Seiten jenes Punktes aus; die Gruppe endigt plötzlich mit den Flächen $V$ und $B$, welche von $b$ um $\frac{1}{2}$ resp. 4 von der gesammten Winkeldistanz $b e$ entfernt sind.

2) Die Stelle, an welche sich die Zone $a v[10 \bar{T}: 20 \bar{T}]$ am meisten $b(2 T T)$ nähert, entspricht einer Fläche, welche $78^{0} 17^{\prime}$ gegen $e(101)$ geneigt 
ist, und dies ist genau jener Punkt, wo die gestreiften Flächen am dichtesten entwickelt sind.

3) Die Stelle, bei welcher die Zone $b q r$ am meisten der Fläche $a$ genähert ist, hal einen Winkelabstand von $12045 \frac{1}{2}^{\prime}$ von $b$, und ihr entspricht eine Ebene, welche in ihrer Lage nahezu mit der Fläche $q$ ubereinstimmt.

In einigen Fällen also ist an jenen Punkten, wo eine Hauptzone eines Pyrargyritkrystalles sich am meisten einer ausserhalb der Zone liegenden Hauptfläche nähert, die Zone gleichsam unstabil und der leichteren Bildung vicinaler, zur selben Zone gehöriger Flächen zugänglich.

\section{§ 19. Isolirte Gruppen vicinaler Flächen.}

Es handelt sich hier um Flächengruppen mit hohen Indices, welche die typischen Flächen ersetzen oder begleiten.

Die oben angeführten, zwischen (23.2.T) und (13.2.T) gelegenen Flächen bilden eine derartige Gruppe. Eine andere wird gebildet von den vier Flächen in der Zone $r \tau[100: 21 \overline{3}]$, welche an dem von Phillips beschriebenen Krystalle die Fläche $i_{3}$ ersetzen - und sich von $Y\{81 \overline{3}\}$ bis $\{61 \overline{3}\}$ erstrecken, wie aus nachstehender Tabelle ersichtlich.

\begin{tabular}{|c|c|c|c|c|}
\hline$r:(h k l)$ & Grenzen : & Anzahl: & Symbol: & Berechnet \\
\hline $20^{0} 44^{\prime}$ & $20^{\circ} 41^{\prime}-20^{\circ} 49^{\prime}$ & 2 & $(81 \overline{3})$ & $20^{\circ} 37^{\prime}$ \\
\hline $24 \quad 28$ & $24 \quad 12-24 \quad 40$ & 2 & $(20.3 . \overline{9})$ & $24 \quad 41$ \\
\hline 2611 & 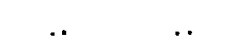 & 1 & $(25.4 .1 \overline{2})$ & $26 \quad 16$ \\
\hline 2729 & $27 \quad 22-2734$ & 2 & $((j \mid \overline{3})$ & 2719 \\
\hline
\end{tabular}

Die Fläche $r(100)$ findet sich an einem Freiberger Krystall begleitet von einem Flächenpaare an den Kanten, welche erstere sie mit zwei Flächen der Form $e\{110\}$ bildel; diese beiden Vicinalfächen bilden mit $r 1^{0} 53^{\prime}$ resp. $201 \frac{1}{2}$, welche Winkel eine genaue Messung gestatteten.

Ein Pyrargyritkrystall von Andreasberg mit den Endflächen epr zeigt vier Flächen, welche die Kanten $e p$ ersetzen und folgende Neigungen gegen $e$ haben :

$\begin{array}{ccccc}e:(h k 0) & \text { Grenzen : } & \text { Zahl : } & \text { Symbol: } & \text { Berechnet: } \\ 2027^{\prime} & 2024^{\prime}-2^{0} 30^{\prime} & 2 & (71.63 .0) & 2^{0} 27^{\prime} \\ 254 & 253-254 & 2 & (61.53 .0) & 253 \\ 330 & 326-335 & 2 & (51.43 .0) & 330 \\ 410 & 411-419 & 2 & (41.33 .0) & 426\end{array}$

Diese Reihe schreitet zweifelsohne um gleiche Incremente nicht der Winkel, sondern der Indices fort.

An einem Proustitkrystalle von Chañarcillo wird das Skalenoëder $v\{207\}$ begleitet von kleinen Facetten in der Zone $a v[10 \bar{T}: 20 \bar{T}$, wovon drei uber $v$ gegen $r$ ur.d drei darunter gegen $a$ liegen. Jene uber $v$ gelegenen sind 
gegen $v$ unter den Winkeln $2^{0} 53^{\prime}, 3^{0} 18^{\prime}, 3^{0} 36^{\prime}$ und jene unter $v$ unter den Winkeln 1039', $1054^{\prime}$ und $2050^{\prime}$ geneigt.

An einem Pyrargyritkrystalle von Andreasberg ist am aufgewachsenen Ende des Krystalles die Fläche $n\{40 T\}$, welche $50^{\circ} 8^{\prime}$ gegen das Prisma $a\{10 \bar{T}\}$ geneigt ist, begleitet von vier Flächen in der Zone $n a$, welche gegen

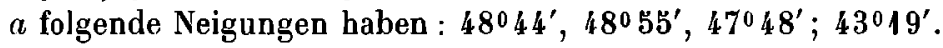

An einem Pyrargyritkrystalle von Andreasberg ist die Fläche von $r$ am aufgewachsenen Ende begleitet von zwei Rhomboëderflächen, zu derselben verticalen Reihe gehörig und gegen $r$ geneigt unter den Winkeln :

$$
\begin{array}{ll}
6^{0} 23^{\prime} & r:(14 . T . T)=6^{0} 231^{\prime} \\
7^{0} 15^{\prime}-7046^{\prime} & r:(12 . T . T)=729 \frac{1}{2}
\end{array}
$$

\section{§ 20. Vertheilung der Flächen.}

Die Hauplzonen; welche fur das obere und untere Ende eines Pyrargyritkrystalles charakteristisch sind, sind gegeben durch die Kanten einer Combination des Prismas $a$ und des trigonalen Prismas $b$ mit den Flächen $r e v$ am oberen und $r e n$ am unteren Ende, wie sie in Fig. 2 dargestellt sind.

Die charakteristischen Zonen des oberen Endes sind:

$\begin{array}{lcll}{[a v r e],} & \text { typische Flächen: } & N^{\prime} y \gamma v \rho w t \lambda p, \\ {[b e]} & - & - & V x^{\prime} L B, \operatorname{mit} g F^{\prime} A \Xi \sigma, \\ {[v v]} & - & - & q f E T, \\ {[v v]} & - & - & \alpha s, \\ {[b r]} & & - & T R u s \Gamma, \text { mit } I, \\ {[b v]} & - & - & X l d \text { mit } p^{\prime} \varrho .\end{array}$

Die charakteristischen Zonen des unteren Endes sind :

[br] typische Flächen: $D q F E$ mit $G H N$ u.s. w.,

$\begin{array}{llll}{[a n r e]} & - & - & v n t p-s^{\prime} \psi w^{\prime}, \\ {[n n]} & - & - & \alpha \mathbf{Y} \boldsymbol{\Pi}, \\ {[b e]} & - & - & f u .\end{array}$

Aus der Projection (s. Taf. IV) ist zu ersehen, dass die Pole meistens auf Zonen mit einfachen Symbolen liegen, welche von den Polen der Flächen $r$ und $e$ ausstrahlen.

Die von Goldschmidt (Index 3, 59f.) gegebenen Bezeichnungen $G_{1}$ und $G_{2}$ können als diejenigen von Flächen betrachtet werden, welche zu Reihen in Zonen gehören, deren Radiationspunkte Flächenpaare der Prismen $a$ und $b$ sind. Die Symbole $p q$ der Bezeichnung $G_{1}$ Jeiten sich vom Mille r'schen Symbol $(h k l)$ ab durch die Relationen:

$$
p=\frac{h-k}{h+k+l}, \quad q=\frac{k-l}{h+k+l} .
$$


Man kann eine ähnliche Bezeichnung anwenden, um die Flächen als Schnittpunkte von Zonen, welche von $r$ und $e$ ausstrahlen, auszudrucken; nimmt man nämlich fur das Symbol $p q$ die Ausdrücke:

$$
p=\frac{k}{-l}, \quad q=\frac{h-k+l}{-l}
$$

so findel man, dass fast alle sicher festgestellten Formen durch sehr einfache Zahlen, einfachere als in den Goldschmidt'schen Symbolen, bezeichnet werden.

\section{§ 21. Chemische Zusammensetzang.}

Im Nachstehenden wurde der Versuch gemacht, die Krystallform und die chemische Zusammensetzung sowohl der reinen Verbindungen Pyrargyrit und Proustit, als auch der Arsen- resp. Antimon-haltigen Varietäten zu ermitteln. Zu diesem Zwecke wurden nach dem in $\$ 4$ erwähnten Verfahren aus der Sammlung des British Museum 15 Krystalle ausgesucht und von Herrn G. T. Prior analysirt.

Die Analysen wurden nach der, in dieser Zeitschr. 14, 114 gegebenen Methode ausgefuhrt. Das Mineral wurde stets durch einen Chlorstrom zersetzt.

In jenen Fällen, wo beim Pyrargyrit geringe Mengen von Arsen von einem bedeutenden Antimongehalt zu trennen waren (mit Ausnahme von Nr. 10), wurde die von $\mathrm{Hufschmidt}$ und Clas sen modificirte Fischersche Melhode angewendet, während beim Prouslil geringe Mengen Antimon von dem grösseren Arsengehalt vermittelst des Magnesiagemisches getrennt wurden. Bei fast allen Analysen wurde der Schwefel in einer besonderen Probe bestimmt.

Der Silbergehalt wird durch die Zersetzung des Minerals im Chlorstrome bei langsamem und vorsichtigem Verfahren mit grosser Genauigkeit ermittelt.

Er kann deswegen, weil, wie die nachstehenden Analysen zeigen, alle untersuchten Krystalle (mit Ausnahme von Nr. 2) aus Mischungen von Pyrargyrit- und Proustitsubstanz bestehen, als bestimmend fur die $\mathrm{Zu}-$ sammensetzung irgend eines Exemplares angesehen werden. Aus diesem Grunde, und weil durch die Fresenius-B abo'sche Methode kein Arsen gefunden wurde, ist von $\mathrm{Nr}$. 2 allein die Silberbestimmung gegeben.

Fischer's Trennungsmethode von Arsen und Antimon scheint vortrefflich zu sein, wenn der Arsengehalt im Verhältniss zum Antimongehalt beträchtlich geringer ist; anderen Falles war gewöhnlich noch eine zweite Destillation nöthig, um eine vollständige Trennung zu bewirken. Aus diesem Grunde wurde auch beim Proustit die Trennung mit der Magnesia- 
mischung durchgefuhrt. Es scheint jedoch, als ob diese Methode nicht immer eine vollkommene scharfe Trennung ermöglicht, da Spuren von Arsen beim Auswaschen mitgehen können. Der Antimongehalt ist deshalb bei den Proustilanalysen wahrscheinlich etwas zu hoch, jedoch keinenfalls mehr als $0,1 \%$.

Von den zwei angefuhrten Rhomboëderwinkeln ist der erste stels der gemessene, der zweite der aus diesem berechnete.

\section{Pyrargyrit.}

1) Catalog Nr. 29709. Angewendetes Material 1,6968 g. Specifisches Gewicht 5,82.

$$
\begin{array}{clcc}
A g & \ldots & 59,75 \\
S & \ldots & 17,81 \\
& S b & \ldots & 22,45 \\
& & & \frac{100,01}{100} \\
\text { Rhomboëderwinkel } & e: e=4205^{\prime} & \left(4204^{\prime} 37^{\prime \prime}-42^{\circ} 5^{\prime} 35^{\prime \prime}\right) \\
& r: r=7122
\end{array}
$$

Andreasberg. Typisches Exemplar; Habitus der Seitenflächen flach, der Endigung pyramidal. Combination $t v e a b p \nu \lambda \omega$ u.s.w., $p$ ist glatt und glänzend. Gruppe glänzender Krystalle auf Galcit; Zwillinge nach $u$.

2) Catalog Nr. 48555. Angewendetes Material 1,4511 g. Specifisches Gewicht 5,85 .

$$
\begin{gathered}
A g \quad \ldots 59,74 \% \text { (enthält kein Arsen). } \\
\text { Rhomboëderwinkel } \quad e: e=4204 \frac{1}{2} \\
r: r=7122
\end{gathered}
$$

Grube Santa Lucia, Guanaxuato, Mexico. Typisches Exemplar. Habitus der Seitenfläche prismatisch, Endigung flach (s. §4). Gruppe glänzender Prismenzwillinge nach $a$.

3) Catalog Nr. 37042. Angewendetes Material 0,8920 g.

$\begin{array}{llr}A g & \ldots & 59,91 \\ S & \ldots & 17,79 \\ S b & \ldots & 22,09 \\ A s & \ldots & 0,12 \\ & & 99,91\end{array}$

$$
\begin{array}{ll}
\text { Rhomboëderwinkel } & r: r=71022^{\prime} \\
& e: e=42 \quad 5
\end{array}
$$

Andreasberg (Grube Abendröthe?); Habitus der Seitenflächen skalenoëdrisch; Endigung rhomboëdrisch; Combination von ayVLeXbm' $\omega p t w \varphi$ $A B r$ (ohne $e$ und $v)$. Glänzende Krystalle auf Calcit. 
4) Catalog Nr. A.G. 20. Angew. Material 1,4139. Spec. Gew. 5,83.

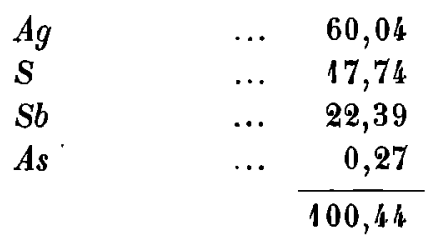

Galega Mine, Zacatecas, Mexico. Habilus prismatisch; Combination $a b u$ mit $q r n$ am aufgewachsenen Ende. Gruppe grosser, matter, unebener Krystalle, Zwillinge nach $a$.

5) Catalog Nr. $37042 \cdot(3)$. Angew. Material 1,6977 g.

\begin{tabular}{lr}
$A g$ & 57,46 \\
$S$ & 18,62 \\
$S b$ & 23,73 \\
$A s$ & 0,30 \\
\hline & 100,11
\end{tabular}

Rhomboëderwinkel $\quad r: r=71022^{\prime}$

$$
e: e=42 \quad 5
$$

Andreasberg. Habitus der Seitenflächen lanzettförmig. Endigung rhomboëdrisch; Combination von $\Gamma q F a X r V L$ eic. Schöne Krystallgruppe mit Bleiglanz und Miargyrit auf Calcit. Die seitlichen Flächen sind mit Krystallen von Hypargyrit bedeckt, welche sie theilweise durchdringen.

Die Analyse ist ohne Zweifel durch den im gepulverten Material erkennbaren Hypargyrit beeinflusst. Eine Miscbung von $10 \%$ Miargyrit mit $90 \%$ Pyrargyrit wurde folgende Zusammensetzung haben :

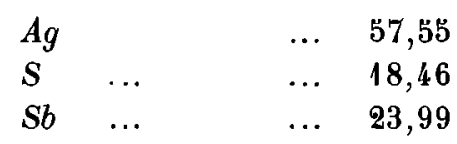

6) Catalog Nr. 57648 . Angew. Material 2,0966 g. Spec. Gew. 5,86.

$$
\begin{array}{lr}
A g & 60,24 \\
S & 17,74 \\
S b & 21,69 \\
A s & 0,44 \\
& 100,11
\end{array}
$$

$\begin{array}{ll}\text { Rhomboëderwinkel } & r: r=71010^{\prime} \\ e & : e=4155 \frac{1}{2}\end{array}$

Andreasberg. Habitus der Seitenflächen lanzettförmig. Endigung rhomboëdrisch, beinahe pyramidal ; Combination von aFybqXLtre $p x^{\prime} B p \gamma Q p^{\prime}$ 
mil $r n \Pi s^{\prime}$ am aufgewachsenen Ende, s. Fig. 8. Habitus ähnlich dem von 3) und 5) und dem Phillip s' schen Krystalle. Glänzende, drusige Krystalle mit Bleiglanz auf Calcit.

7) Catalog Nr. 29714 . Angew. Material 0,9591 g. Spec. Gew. 5,78.

\begin{tabular}{|c|c|}
\hline$A g$ & 60,17 \\
\hline$S$ & 17,65 \\
\hline$S b$ & 21,64 \\
\hline$A s$ & 0.52 \\
\hline
\end{tabular}

Rhomboëderwinkel

$$
\begin{aligned}
& e: e=420 \quad 8^{\prime} \\
& r: r=71 \quad 25 \frac{1}{2}
\end{aligned}
$$

Freiberg. Habitus der Seitenflächen prismatisch, Endigung rhomboëdrisch. Ein grosser, hohler Krystall mit ein wenig Proustit (?) auf der Oberfäche. Der Winkel wurde an einem kleineren Krystalle (Combination evY) von der Innenseite des Exemplares gemessen.

8) Catalog No. 34 756. Angew. Material 0,9456 g. Spec. Gew. 5,77.

\begin{tabular}{lr}
$A g$ & 60,07 \\
$S$ & 17,89 \\
$S b$ & 21,20 \\
$A s$ & 0,79 \\
\cline { 2 - 2 } & 99,95
\end{tabular}

Chañarcillo. Habitus der Seitenflächen prismatisch. Endflächen rhomboëdrisch. Combination von aeqr etc. Gruppe voll glänzenden Krystallen auf Galcit. Zwillinge nach $u, a$ und $r$.

9) Gatalog Nr. G. v. v. 4. Angew. Material 1,5285 g. Spec. Gew. 5,81.

\begin{tabular}{lr}
$A g$ & 60,21 \\
$S$ & 17,78 \\
$S b$ & 20,69 \\
$A s$ & 1,02 \\
\cline { 2 - 2 } & 99,70
\end{tabular}

Harz. Habitus der Seilenflächen prismatisch; Endflächen pyramidal. Combination von at mit $\alpha r n v q u$ am anderen Ende, s. Fig. 3. Zwillinge nach $a$. Grosse, unebene, an beiden Enden ausgebildete Krystalle mit Galcit, Bleiglanz, Arsenkies (?) und wenig Fablerz. Die Krystalle sind für genaue Messungen *) zu rauh, auch sind sie etwas lichter gefärbt, was aber durch die drusige Beschaffenheit bedingt sein mag.

*) Zwei Kanten ergaben genaue Messungen $v: v=35^{015^{\prime}}$ und $n: n=17^{0} 37^{\prime}$; die erste führt auf $e: e=42^{0} 15^{\prime}$, die zweite auf $e: B=41^{0} 19^{\prime}$. 
10) Catalog Nr. 34 435. Angewendeles Material 1,3223 g. Specifisches Gewicht 5, 805 .

$$
\begin{array}{lllr}
A g & \ldots & \ldots & 60,85 \\
S & \ldots & \ldots & 17,99 \\
S b & \ldots & \ldots & 18,36 \\
A s & . . & \ldots & \frac{2,60}{} \\
& & & 99,80
\end{array}
$$

Rhomboëderwinkel

$$
\begin{aligned}
& r: r=71030^{\prime} \\
& e: e=4211 \frac{1}{2}
\end{aligned}
$$

Andreasberg. Kleine Gruppe glänzender, hellrother Krystalle; Habitus und Combination wie in Fig. 4 dargestellt. Prismenflächen glatt, die $e$-Fläcben dagegen uneben und concav. Diese Krystalle bilden einen Ueberzug auf einer derben, drusigen Masse, von der sie theilweise durch drusige Hohlräume getrennt sind und welche aus winzigen Krystallen mit den Flächen $a e s v y t$ besteht. Der Rhomboëderwinkel konnte nicht direct gemessen werden, sondern ist berechnet aus den Messungen $v: v=74^{0} 27^{\prime}$ und $a: v=24^{\circ} 51^{\prime}$, weswegen demselben keine grosse Bedeutung beigelegt werden kann. Die Analysen sind mit Material ausgefuhrt, welches sowohl dem krystallinischen Ueberzug, als der drusigen Masse angehört, da es nicht möglich war, von beiden Theilen genug Material zu erlangen, ohne die kleine, nur $7 \mathrm{~g}$ wiegende Gruppe zu zerstören. Der Strich auf Papier ist derjenige des Pyrargyrit, während die Farbe des Pulvers in einem Glasröhrchen elwas lichter erscheint.

\section{Proustit.}

1) Gatalog Nr. 26751 . Angewendetes Material 1,4103 g.

$$
\begin{array}{lll}
A g & \ldots & 6 \ddot{3}, 39 \\
S & \ldots & 19,52 \\
A s & \ldots & 14,98 \\
\hline & & 99,89
\end{array}
$$

Rhomboëderwinkel $\quad e: e=42^{0} 46^{\prime}$

$$
\left.r: r=72 \quad 12 \text { (beobachlet } 72^{0} 6^{\prime}\right) \text {. }
$$

Mexico: Derb und in gläuzenden Krystallen auf Calcit. Combination von a vrew $\Psi M d s$, s. Fig. 7. Zwillinge nach $r$.

2) Catalog Nr. 39862 . Angewendetes Material 1,4569 g. Specifisches Gewicht 5, 59. 


\begin{tabular}{|c|c|c|}
\hline$A g$ & & 65,37 \\
\hline$S$ & & 19,24 \\
\hline$A s$ & $\ldots$ & 14,81 \\
\hline $\mathbf{S b}$ & $\ldots$ & 0,59 \\
\hline
\end{tabular}

Der Antimongehalt ist zweifellos etwas zu hoch.

Rhomboëderwinkel $\quad e: e=42^{\circ} 46^{\prime}$
$r: r=7912$

Ghañarcillo. Glänzende, skalenoëdrische Bruchstucke. Combination von $a$ ves $\boldsymbol{M} \boldsymbol{w} \boldsymbol{\Psi}$.

3) Calalog Nr. 35 832. Angewendetes Material 1,9635 g. Specifisches Gewicht 5,58.

\begin{tabular}{lrr}
$A g$ & & 65,38 \\
$S$ & & 19,31 \\
$A s$ & & 14,89 \\
$S b$ & & 0,26 \\
\hline & & 99,84
\end{tabular}

Rhomboëderwinkel $\quad e: e=42045 \frac{1}{2}^{\prime}$

$r: r=7212$

Chañarcillo. Glänzende, lose Krystalle, Combination von a ve etc. Zwillinge nach $u$ und $r$.

4) Calalog Nr. 62 322. Angewendetes Material 1,2936 g. Specifisches Gewicht 5,64.

$$
\begin{array}{llr}
A g & \ldots & 65,06 \\
S & \ldots & 19,64 \\
A s & \ldots & 13,85 \\
S b & \ldots & 1,41 \\
\hline & & -\frac{99,96}{}
\end{array}
$$

Rhomboëderwinkel $\quad e: e=42^{\circ} 46^{\prime}$

$r: r=72 \quad 12$

Chañarcillo. Glảnzende Krystalle auf Calcit, Combination $e v s$. Theile dieses Stuckes enthalten nach der früheren ausfuhrlichen Beschreibung (diese Zeitschr. 14, 113) 3,53\% Antimon.

5) Catalog Nr. 34423.

$$
\begin{array}{lllr}
A g & \ldots & & 64,43 \\
S & \ldots & & 19,54 \\
S b & \ldots & \ldots & 3,74 \\
A s & \ldots & \ldots & \frac{12,29}{100,00} \text { (aus der Differenz) }
\end{array}
$$


Sachsen (?). Grosse unebene prismatische Krystalle von Proustit, mit Pyrargyrit uber\%ogen; Combination aeq, mit Speiskobalt, Calcit, Pyrrhotin und Fluorit vergesellschaftet. Zur Analyse wurden die inneren Theile des Exemplares verwendet. Ein kleines aus der Oberfläche hervorragendes Kryställchen gab eine approximative Messung $e: e=41^{\circ} 24^{\prime}$.

\section{§ 22. Beziehnngen zwischen Znsammensetzung, Form, Art des Vorkommens ete.}

Die Schwankungen des Rhomboëderwinkels der sämmtlichen untersuchten Pyrargyritkrystalle liegen innerbalb der Grenzen der Schwankungen an einzelnen Exemplaren, stehen also mit den Differenzen des Arsengehaltes nicht in Verbindung.

Der Rhomboëderwinkel des reinen, arsenfreien Pyrargyrits (s. §7) ist gleich 71022'; jener der arsenhaltigen (von einer Spur bis 2,6\%) Varietäten nach derselben Methode bestimmt, ergab sich zu:

$\begin{array}{cccc}r: r & \text { Zahl der Kanten: } & \text { Grenzen von } r: r & \text { Grenzen von } e: e \\ 710221^{\prime} & 108 & 71010^{\prime} \text { bis } 71026^{\prime} & 41055^{\prime} \text { bis } 42^{\circ} 8^{\prime} .\end{array}$

Dieses Resultat grundet sich auf die directe Messung der Rhomboëderwinkel von 36 Krystallen, welche 18 Handstücken entnommen wurden, deren Fundorte sind: Andreasberg, Laasphe, Freiberg, Schneeberg, Bräunsdorf, Hiendelaencina, Guanaxuato, Zacatecas und einige von unbekannten Fundorten.

Das Vorhandensein von Arsen wurde in säınmtlichen Exemplaren mittelst der Fresenius-Babo'schen Methode nachgewiesen.

Eine bestimmte Beziehung zwischen dem Arsengehalte des Pyrargyrits und dem Habitus oder Ansehen der Krystalle existirt nicht. Krystalle von völlig identischem Aussehen enthalten zuweilen eine Beimengung von Arsen, zuweilen sind sie frei davon, während mancher Pyrargyrit von merklich hellerer Farbe arsenfrei gefunden wurde.

Die 22 untersucbten Exemplare von reinem Pyrargyrit zeigen Krystalle von allen vier Arten des in $§ 5$ erwähnten Habitus der Seitenflächen, mit pyramidaler und rhomboëdrischer Endigung. Sie stammen von folgenden Fundorten: Andreasberg, Gonderbach, Freiberg, Hiendelaencina, Guanaxuato, Zacatecas und Peru; Begleitmineralien sind: Calcit, Galenit, Stephanit, Quarz, Stibnit, Kupfer, Pyrit, Chalybit, Baryt, Blende und in zwei Fällen aus dem Harz Arsenik.

Die an denselben beobachteten Formen sind:

$$
\text { evatbpv } \lambda \omega y \text { UrfPsuqw } \vartheta \alpha \text {. }
$$

Die Pyrargyrite von Hiendelaencina, sowie die meisten von Laasphe sind merklich arsenfrei. 


\section{Speciflsches Gewicht.}

Die nachstehend zusammengestellten Bestimmungen sind meistentheils scbon in $\S 21$ angeführt.

$$
\text { Pyrargyrit. }
$$

Sp. Gew.: Ang. Substanz: Melhode:

1) $\left\{\begin{array}{l}5,86 \\ 5,85\end{array}\right.$

$5,0 \mathrm{~g}$ Pyknometer

Arsenik :

Winkel $e: e$

2) 5,85

$2,2-$

Hydrostatisch

0

$42^{0} \quad 4 \frac{1}{2}^{\prime}$

3) 5,85

4,0 -

Pyknometer

22, 2 -

Hydrostatisch

$\cdots$
0

4) 5,84

24,0 -

5) 5,84

$6,0-$

6) 5,84

44,8 -

7) $\left\{\begin{array}{l}5,82 \\ 5,82\end{array}\right.$

$4,0-$

8,8 -

-

- Geringer $\% / 0^{-G e h a l t}$

Spur

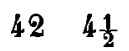

426

8) 5,83

2,5 -

Pyoter

0

9) 5,86

0,8 -

Fydrostatisc
Pyknometer

0

425

10) 5,80

$1,9-$

$0,27 \%$

0,44 -

4156

11) 5,81

5,0 -

2,60 -

12) 5,78

10,4 -

$1,02-$

13) 5,77

2,5 -

$0,52-$

428

In vorstehender Tabelle ist :

1) Sehr schöner Krystall der Combination atevys $P u q$, von Samson.

2) Material der Analyse 2.

3) Schöner Krystall der Combination paveybs $Z$ Pq, von Samson.

4) Schöner Krystall der Combination tve yabeqnG.

5) Grosser, hohler, skalenoëdrischer Krystall vom Habitus des Proustit, Freiberg.

6) Lichtgefärbte Masse von Krystallen, avt, vom Harz.

7) Material zur Analyse 1.

8 8) - $\quad-4$.

9) $-\quad-\quad-6$.

10) - $\quad-\quad 10$.

11) - $\quad-\quad 9$.

12) - - - 7 .

13) - - $\quad-8$. 


\section{Proustit.}

Sp. Gew.: Ang. Substanz: Methode:

1) 5,55

2) 5,57

3) $\quad 5,58$

4) 5,59

5) 5,59

6) 5,60

7) $5,6 \mathbf{2}$

8) ... 5,64

\section{$7,7 \mathbf{g}$}

$0,9-$

$2,7-$

2,3

$4,5-$

15,3 -

$50,5-$

$2,4-$
Hydrostatisch

Pyknometer

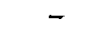

Hydrostatisch

Pyknometer
Antimon: Winkel $e: e$

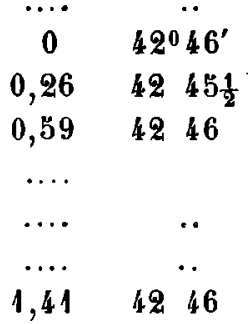

In dieser Tabelle ist:

1) Schöne Gruppe. abgebildet in Fig. 27. Chañarcillo.

2), 3), 4) Material der Analyse resp. 1, 3, 2.

5) Klare Bruchstücke von Chili.

6) Schöne Gruppe, Combination vseu. Chañarcillo.

7) Grosse Krystallmasse, Combination ta e. Andreasberg.

8) Material zur Analyse 4.

\section{Inhalt:}

$\S$ 1. Historische Uebersicht, Literatur . . . . . . . . . . . . . . 129

2. Resultate...................... 132

$\S$ 3. Beschreibung des Pyrargyrit und Proustit ............... 134

$\S$ 4. Farbe und Strich . . . . . . . . . . . . . . . . . . 135

5. Habilus ..................... 137

$\S$ 6. Bestimmung des Rhomboëderwinkels . . . . . . . . . . 137

7. Festgestellte Formen. . . . . . . . . . . . . . . . 140

8. Ausscheidung unsicherer Formen . . . . . . . . . . . . . . . . . . . 144

\$. Kritik der krystallographischen Angaben früherer Autoren . . . . . . \$45

§10. Messungen und Charakter der Flächen . . . . . . . . . . . . . . . 149

§11. Neue Formen: a) sichere, b) zweifelhafte . . . . . . . . . . . . 151

\$1. Formen, welche dem Pyrargyril und Proustit gemeinsam sind . . . . . 156

§13. Relative Häufigkeit der Formen . . . . . . . . . . . . . 156

14. Typische Formen . . . . . . . . . . . . . . . . . 1.57

15. Rhomboëdrischer Charakler des Rolhgiltigerzes . . . . . . . . 158

\$16. Hemimorphismus . . . . . . . . . . . . . . . 159

17. Zwillingsverwachsungen .................. 162

$\S$ 18. Hauptzonen (gekrümmte und gestreifte): a) Beobachtungen, b) Indices der Flächen in gestreiften Zonen, c) Positition gekrümmter und gestreifter

Flächengruppen .................... 178

§19. Isolirte Gruppen vicinaler Flächen . . . . . . . . . . . . . . . . . . . 182

$\S$ 20. Allgemeine Vertheilung der Flächen . . . . . . . . . . . . . . . . . 183

21. Chemische Zusammenselzung. . . . . . . . . . . . . . . . . . . 184

622. Beziehungen zwischen chemischer Zusammensetzung, form, Art des Vor-

kommens etc. . . . . . . . . . . . . . 190

§23. Specifisches Gewicht. . . . . . . . . . . . . . . . 191 


\section{Erklärung der Tafeln.}

Tafel IV.

Stereographische Projection der typischen Formen; rechts die des Pyrargyrit, links die des Proustit.

Fig. 1. Ideale Combination eines doppelendigen Pyrargyrilkrystalles.

- 2. - , die Hauptzonen erlăuternd.

- 3. Pyrargyrit von Andreasberg, hemimorph.

- 4. - - - -

- 5. - $\quad$ - $\quad$ - $\quad$ - $\quad$ - $\quad$ - $\quad$, die Streifung auf den Prismenflächen

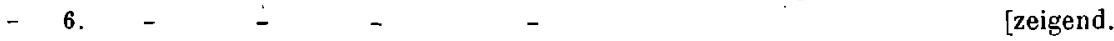

- 7. Proustit von Mexico.

- 8. Pyrargyrit von Andreasberg.

$-9$.

- 10. Typischer Pyrargyritkrystall.

- 11. - Proustilkrystall.

- 12. Proustit von Chañarcillo.

- 13. Pyrargyrit von Mexico, mil Zwillingstafet.

Tafel V.

- 14. Hemimorphes Prisma von Pyrargyrit.

- 15. Fig. 14 in Zwillingsstellung nach der Verticalaxe.

- 16. - 14 - $\quad-\quad$ - $\quad$ - Normale zu $b(10 \overline{1} 0)$.

- 17. - 14 - - - - - - $a(11 \overline{2} 0 ;$

- 18. Verwachsung von 14 und 17, die $q$-Enden nach aussen gerichtet.

- $19 . \quad-\quad-14-17-\quad-\quad$ - innen -

- 20. Fig. 14, in Zwillingsstellung um die Kante $q r$.

- 21. Fig. 14, - - - - eine Axe $\perp$ zu $q r$ ( $u$-Zwilling).

- 22. Verwachsung von 14 und 20.

- 23 . - $-14-21$.

- 24. Zwilling nach $u\{10 \overline{4}\}$, auf einer Seite der Zwillingsebene ausgebildet.

- 25. - - - - zu bciden Seiten der Zwillingsebene ausgebildet.

- 26. Durchwachsungszwilling nach $a$ von Pyrargyrit.

- 27. Zusammengesetzter Zwilling nach $u$ und $r$ von Proustit.

- 28. Proustitzwilling nach $0\{0001\}$. 